2015-08-19

\title{
Geoheritage, a National Inventory in
}

\section{France}

\author{
de Wever, $\mathrm{P}$
}

http://hdl.handle.net/10026.1/4995

10.1007/s12371-015-0151-2

Geoheritage

All content in PEARL is protected by copyright law. Author manuscripts are made available in accordance with publisher policies. Please cite only the published version using the details provided on the item record or document. In the absence of an open licence (e.g. Creative Commons), permissions for further reuse of content should be sought from the publisher or author. 


\title{
GEOHERITAGE,
}

\section{A NATIONAL INVENTORY IN FRANCE}

Patrick De Wever (1), InÈs Alterio (1), Grégoire Egoroff (1), Annie Cornée (1), Peter Bobrowsky (2), Gerard Collin (3), Francis Duranthon (4), Wesley Hill (5), Arnault lalanNe (6), KEVIN PAGE (7)

(1) Géologie, Muséum National d'Histoire Naturelle, 43 rue Buffon CP48, F-75005 Paris,

pdewever@mnhn.fr; ines.alterio@mnhn.fr; geopatrimoine@mnhn.fr; cornee@mnhn.fr

(2) Geological Survey of Canada, 9860 West Saanich Road, Sidney, BC, Canada V8L 4B2, pbobrows@nrcan.gc.ca

(3) Conservateur en Chefdu Patrimoine, ge.collin@orange.fr

(4) CSRPN Midi-Pyrénées \& Muséum d'Histoire Naturelle, 35 Allées Jules Guesde, 31000 TOULOUSE,

francis.duranthon@mairie-toulouse.fr

(5) IUCN WCPA Geoheritage Specialist Group, Secretary general, wesleymhill@ gmail.com

(6) Ministère de l'Écologie, du développement durable, des transports et du logement /DEB/PEM 4, La Grande Arche Paroi Sud, 7eme étage - 92055 la Défense Cedex (arnault.lalanne@developpement-durable.gouv.fr

(7)School of Geography, Earth and Environmental Sciences, Plymouth University, Drakes Circus, Plymouth PLA 8AA, UK

(kpage@plymouth.ac.uk)

\begin{abstract}
Good protection measures for geological heritage should begin with an inventory of geosites. In France, for example, a law enacted in 2002, grants formal recognition to the notion of geological heritage. An inventory and evaluation was then established on a region-by-region basis. By April 2007, the French Ministry of Environment launched the inventory program for the nation's geological heritage and the data are now being collected at a regional scale. The data are being gathered and homogenised, then transferred to the French National Museum of Natural History for examination. The ratified site data are stored and available for public use on a website (http://inpn.mnhn.fr) in a similar structure to natural data that are also processed and stored (flora, fauna, ecosystems, habitats). Today, protecting global heritage is understood as a dynamic process. Instead of placing objects beneath a display case, the conservation approach is now a more modern, active effort, which facilitates access for knowledge and research.
\end{abstract}

KEYWORDS: GEOHERITAGE, FRANCE, EUROPE, INVENTORY, GEOTOURISM, GEOSITES. 


\section{Introduction}

For many years, the protection of "nature" was conducted for cultural reasons. A good example is the case of the Fontainebleau Forest. In 1836, artists, naturalists and hikers succeeded in preventing the cutting of old-growth trees, and the replacement of oak with pine, with the help of the Barbizon's School of Painters (Jean-Baptiste Corot, Jean-François Millet, Théodore Rousseau, Jules Coignet, and others). And no later than 1861, the first nature reserve was created in the Forest, also for artistic reasons. This 1,097 hectares reserve represents one of the first nature reserves to be established in the world, predating Yellowstone National Park which was created in 1872. The former area also became a biological reserve in 1953.

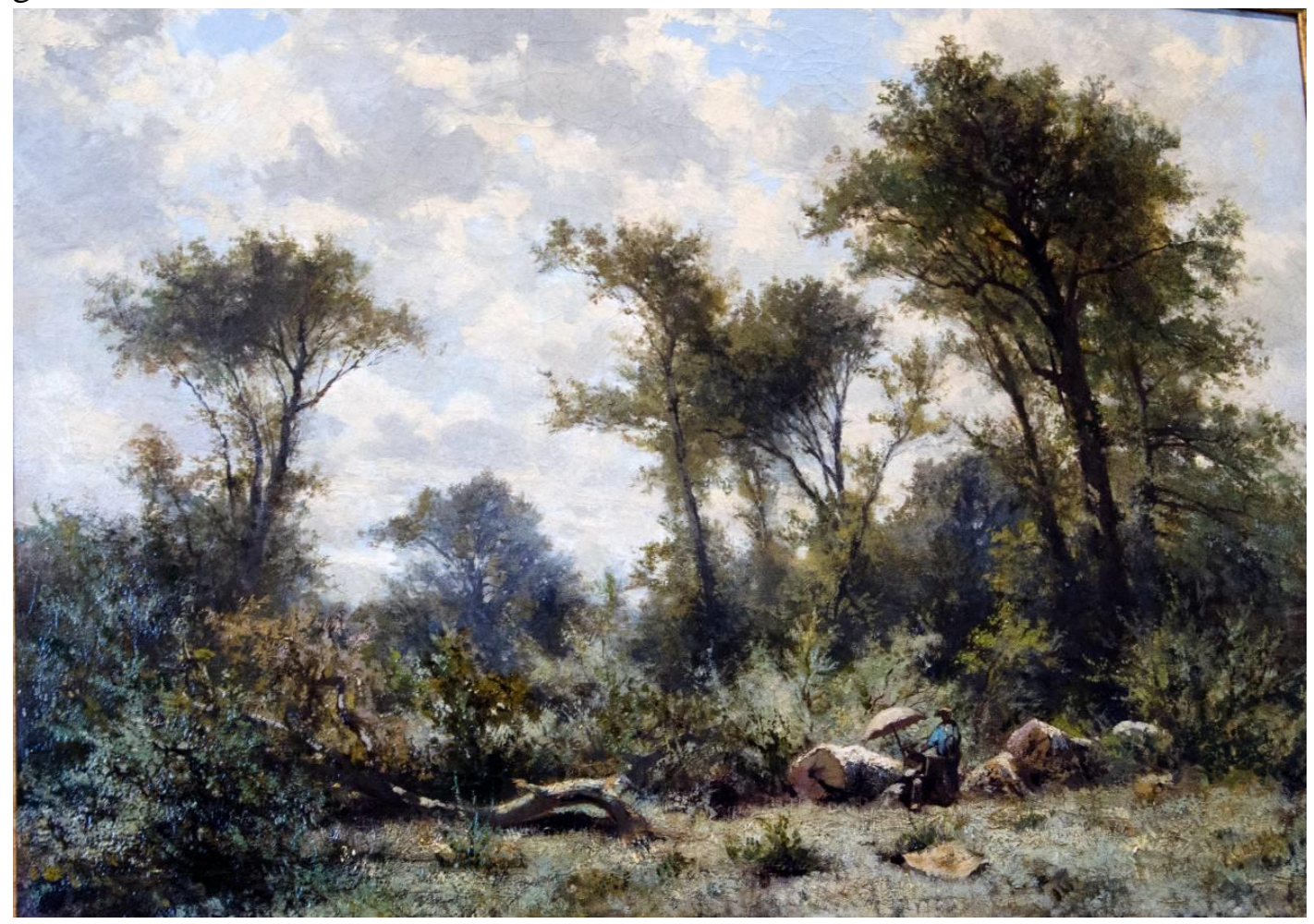

Fig. 1 Barbizon's school of painting: Ernest Cherot (1814-1883), "Peintres sur le motif", oil/canvas (Millet museum, Barbizon, May 2012). Photo: P. De Wever

Over the past 30 years, conservation priority has clearly been directed towards biological heritage. The idea of natural heritage emerged in France with the 1976 law regarding the protection of nature, which officially established the concept of nature reserves.

In this paper we focus on the geological inventory process with a primary focus on France. A general review of geoheritage regulation in France will be described elsewhere.

\section{I- Geological heritage}


Unlike biological conservation, geological conservation has so far lacked a continuously-supported mechanism to recognize and justify the most important elements internationally, i.e. those of the greatest value to the science (Wimbledon, 1996).

\section{What is geoheritage?}

"Si vous voulez converser avec moi, définissez vos termes" ["If you wish to converse with me, first define your terms"] ; Voltaire

The concept of heritage conservation arises from the perception of an external threat. It incorporates the recognition of an eventual threat to an object or a site due to community action, through an economic project or through the use of the resource, and notwithstanding natural threats. Consequently, geoheritage status should, ideally, not be decreed by an extralocal authority, but instead claimed by a local community or the state which has the legal responsibility.

The United Nations Educational, Scientific and Cultural Organization (UNESCO, 1972) considers 'natural heritage' as:

“(1) natural features consisting of physical and biological formations, or groups of such formations, which are of outstanding universal value from the aesthetic or scientific point of view;

(2) geological and physiographical formations and precisely delineated areas which constitute the habitat of threatened species of animals and plants of outstanding universal value from the point of view of science or conservation;

(3) natural sites or precisely delineated natural areas of outstanding universal value from the point of view of science, conservation or natural beauty."

The 1972 general conference of UNESCO further noted that natural heritage was increasingly threatened with destruction not only by the traditional causes of decay, but also by changing social and economic conditions which aggravate the situation with even more formidable phenomena of damage or destruction. It considered that:

"- deterioration or disappearance of any item of the natural heritage constitutes a harmful impoverishment of the heritage of all the nations of the world,

- the existing international conventions, recommendations and resolutions concerning natural property demonstrate the importance, for all the peoples of the world, of safeguarding this unique and irreplaceable property, to whatever people it may belong,

- parts of the natural heritage are of outstanding interest and therefore need to be preserved as part of the world heritage of mankind as a whole,

- in view of the magnitude and gravity of the new dangers threatening them, it is incumbent on the international community as a whole to participate in the protection of the natural heritage of outstanding universal value, by the granting of collective assistance which, although not taking the place of action by the State concerned, will serve as an efficient complement thereto, have decided that this question should be made the subject of an international convention."

An important site of natural heritage can be listed as a World Heritage Site by the World Heritage Committee of UNESCO. The UNESCO programme catalogues, names, and conserves sites of outstanding cultural or natural importance as part of a common heritage of humanity. As of 2014, there were 1,007 World Heritage Sites: 779 cultural, 197 natural and 31 mixed properties, in 161 countries. 
The concept of Geoheritage considers all significant objects (i.e. ex situ geoheritage) and sites (in situ geoheritage) related to the Earth sciences, thus emphasising an interest in a 'memory' of planet Earth. Such objects have to be placed in their natural framework and often represent one or several geological phenomena, or be, to all intents and purposes 'unique', the 'first' described example, or simply the 'best'. The term 'geology; has a wide connotation and includes: palaeontology, mineralogy, tectonics, sedimentology, geomorphology, to name a few included disciplines. Quantifying Geoheritage aims to establish the legacy of geological objects and sites, as well as intangible attributes encompassing the natural environment, landscapes, and landforms which belong to the concept of geodiversity. Heritage is that which is inherited from past generations, maintained in the present and bestowed for the benefit of future generations. The term 'geoheritage' is, therefore, derived from 'geo-inheritance'. It is a less scientific term and may be more easily comprehended by a wider audience interested in geoconservation.

The conservation of geoheritage focuses on preserving those most valued and significant elements and sites, as there are numerous threats that need to be considered such as unsustainable specimen collecting, coastal erosion, quarrying or infill of disused quarries, vegetation overgrowth, urban extension and so on. The concept of geoconservation (the conservation of geoheritage) is a recent phenomenon in most countries, in contrast to France which has had protected natural sites since 1836 (see above) and specifically 'natural monuments' since 1930. In 1913 E.-A. Martel, advocate of the concept of National Park, published a list of important geological sites by county (Martel, 1913). In 1928, he completed his work by publishing La France ignorée, an inventory of remarkable sites containing descriptions and illustrations (Martel, 1928, 1932). Ultimately, National Parks were created in France in 1960 and specific geological reserves were first created in 1982 (Saucats-la-Brède) (Fig. 2).

During the past quarter century, efforts by many geologists can be credited with progressively gaining protection and recognition of several of the geological reserves that were proposed (Fig. 2). As a result of these activities, the 'International declaration of the rights of the memory of the Earth' was written during a seminal international meeting held in Digne-lesBains, Provence, in 1991 (Fig. 3).

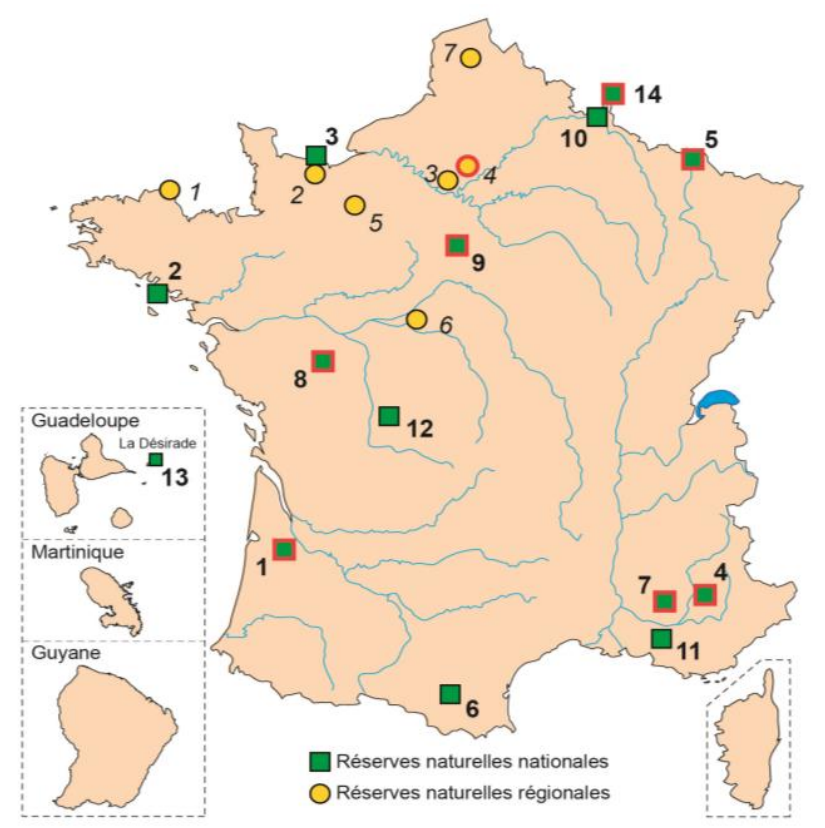


Fig. 2 Map of the reserves created for geological reasons in France. Those with a stratotype have a red border.

- Réserves naturelles nationales. 1) Saucats-La Brède (Aquitanian - Burdigalian). 2) François le Bail (Ile de Groix). 3) Falaise du Cap Romain. 4) Haute Provence (Barremian). 5) Hettange-Grande (Hettangian). 6) Grotte du TM 71. 7) Luberon (Aptian). 8) Thouars (Toarcian). 9) Sites géologiques de l'Essonne (Stampian). 10) Vireux-Molhain. 11) SainteVictoire. 12) Astroblème de Rochechouart-Chassenon. 13) La Désirade. 14) La Pointe de Givet: this reserve was not initially created for geological reasons, but it encloses the historical Givetian stratotype.

- Réserves naturelles régionales. 1) Sillon de Talbert. 2) Anciennes carrières d'Orival. 3) Site géologique de Limay. 4) Site géologique de Vigny-Longuesse. 5) Normandie Maine. 6) Pontlevoy. 7) Anciennes carrières de Cléty.

International declaration of the rights of the memory
of the Earth
1 - Just as human life is recognized as being unique, the time has come to
recognize the uniqueness of the Earth.
2 - Mother Earth supports us. We are each and all linked to her, she is the link
between us.
3 - The Earth is 4,5 billion years old and the cradle of life, of renewal and of the
metamorphosis of life. Its long evolution, its slow rise to maturity, has shaped
the environment in wich we live.
4 - Our history and the history of the Earth are closely linked. Its origins are our
origins, its history is our history and its future will be our future.
5 - The aspect of the Earth, its very being, is our environment. This environment
is different, not only from that of the past, but also from that of the future. We
are but the Earth's companion with no finality, we only pass by.
6 - Just as an old tree keeps all the records of its growth and life, the Earth
retains memories of its past... A record inscribed both in its depths and on the
surface, in the rocks and in the landscapes, a record which can be read and
translated.
7 - We have always been aware of the need to preserve our memories - i.e. our
cultural heritage. Now the time has come to protect our natural heritage, the
environment. The past of the Earth is no less important than that of human
beings. Now it is time for us to learn to protect, and by doing so, to learn about
the past of the Earth, to read this book written before our advent: that is our
geological heritage.
8 - We and the Earth share our common heritage. We and governments are but
the custodians of this heritage. Each and every human being should understand
that the slightest depredation mutilates, destroys dans leads to irreversible losses.
Any form of development should respect the singularity of this heritage.
9 - The participants of the 1st internationl symposium on the protection of our
geological heritage, including over a hundred specialists from over thirty
nations, urgently request all national and international authorities to take into
consideration and to protect this heritage by means of all the necessary legal,
financial and organizational measures.
Written on the 13th June 1991 in Digne, France.

Fig. 3 The International declaration of the rights of the memory of the Earth (Digne-les-Bains, 1991) 
The concept of biodiversity conservation and our need to protect biological heritage at local, national and global scales is well developed. The concept of geodiversity and geoheritage conservation, however, is less appreciated, although it is now beginning to develop some momentum. At a global level, a number of initiatives are now beginning to establish that geoheritage is an important element in our natural heritage and must be managed effectively.

\section{The World Heritage Convention}

The World Heritage Convention (1972) is unique in two respects. Firstly, it recognises both natural and cultural heritage, and secondly it provides a global mechanism for identifying and protecting important geological sites. The Convention promotes, at the global level, a wide range of geological heritage features- from small sites a few hectares in size to large areas within protected landscapes. There is considerable scope for developing new ideas and new ways of recognising outstanding geological and geomorphological heritage, and linking it with cultural and natural heritage values. To better understand how the World Heritage Convention might recognise geoheritage in the future, both in its own right and as a complement to other natural and cultural values, the International Union for Conservation of Nature (IUCN), the advisory body to the UNESCO World Heritage Committee on natural heritage, undertook a thematic study of the role of geology and geomorphology in the World Heritage Convention (http://whc.unesco.org/en/convention/).

The United Nations General Assembly proclaimed 2008 to be the International Year of Planet Earth and the effort was jointly initiated by the International Union of Geological Sciences (IUGS) and UNESCO. In its two latest sessions, the General Assembly of IUCN accepted that geodiversity, including geological and geomorphological diversity, is an important natural factor underpinning biological, cultural and landscape diversity (Resolutions WCC-2008-Res-040 and WCC-2012-Res-048: 2008 Barcelona: https://portals.iucn.org/library/efiles/documents/WCC-4th-005.pdf; 2012 Jeju: https://portals.iucn.org/library/efiles/documents/WCC-5th-005.pdf).

\section{II- Socio-economic, socio-cultural dimensions of geoheritage}

\section{What we call geoheritage}

Geology should be considered in a relationship with nature, culture and history - all of which permanently interact. Humans wish to understand the geographical, natural and socioeconomic context in which they develop, and this cannot be successfully addressed in the absence of geosciences. Indeed the history of human-kind, animals, and plants is tightly connected with the Earth's history. Landscapes, agricultural and commercial practices, including ancient industries, are all firmly dependent on the nature of the Earth (soil and bedrocks), and its resources. In today's practices, local becomes global, and the effect of geology on the environment is also becoming global (natural resources, energy, water, etc) with natural hazards, pollution and climate change examples of such widespread impact. 
What we term geoheritage here, are the geological features and sites with global, national, or local importance and that represent processes (magmatic segregation, metamorphism, dissolution, weathering, etc) or a testimony of the Earth's history (palaeontology, global tectonics, evidence of sea level change, etc). Geoheritage-related objects at any scale (country size to mineral size) are intrinsically or culturally important. They offer information or insights into the formation or evolution of the Earth, into the history of science, or can be used for research, reference, educational purposes or other societal purposes, such as artistic or spiritual inspiration.

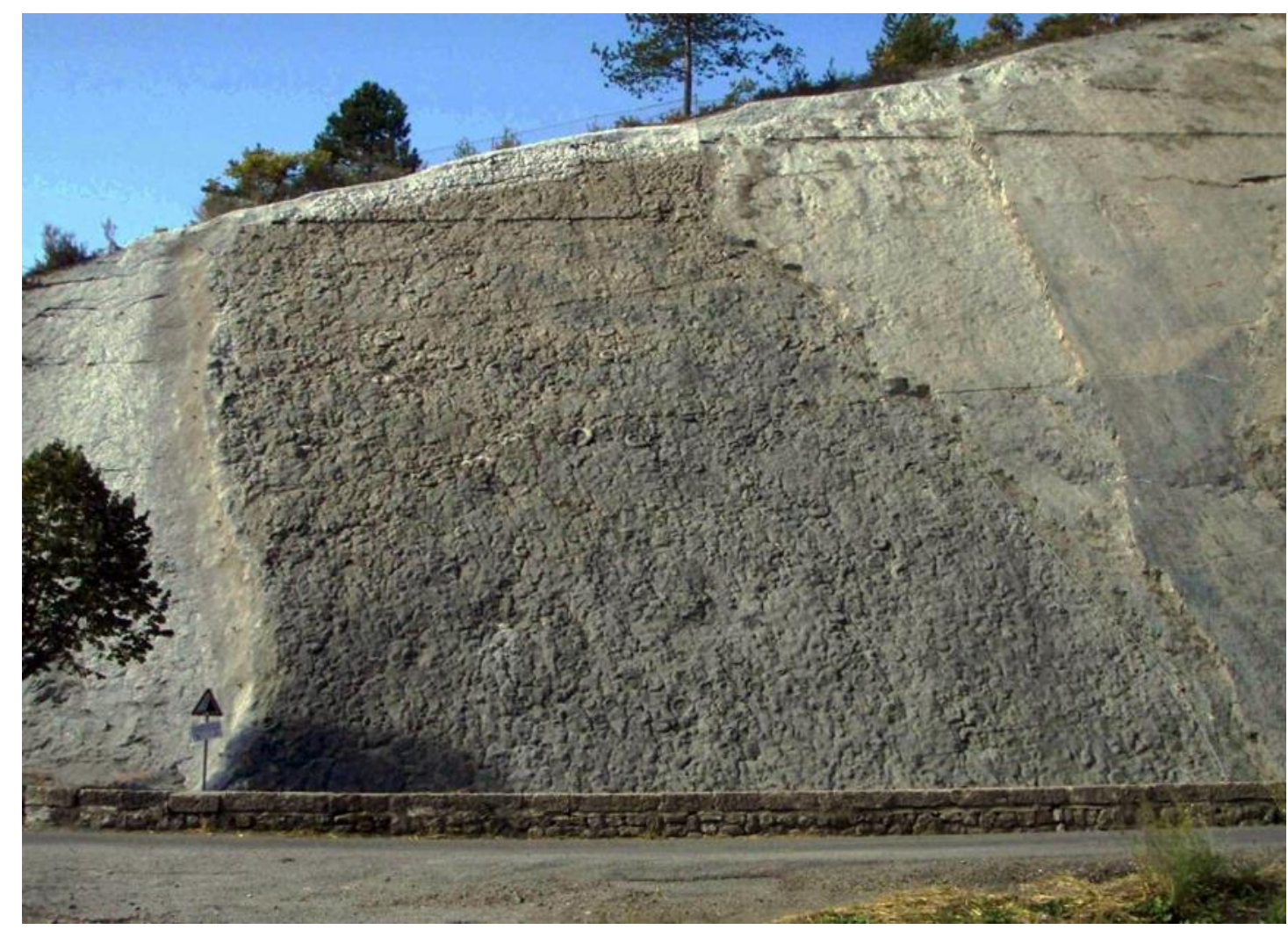

Fig. 4. The famous ammonite slab in the Digne-lel-Bains geological reserve presents more than 1,500 ammonites, up to $70 \mathrm{~cm}$ in diameter, of Lower Sinemurian (Lower Jurassic) age, most belonging to Coroniceras ex grp. multicostatum (J. Sowerby). (Photo M.Guiomar, 2009)

\section{What belongs to heritage?}

We refer to heritage when we discuss items that belong to human-kind in a more global sense in contrast to 'patrimonial [i.e. heritage] value', when we refer to what is selected. Some objects, because their exemplarity or scarcity automatically belong to a 'geoheritage', such as the famous 'Ammonites slab' in the Réserve géologique de Haute Provence (Fig. 4) or some outcrops from South Morocco rich in Devonian orthocone nautiloids (Fig. 5). 


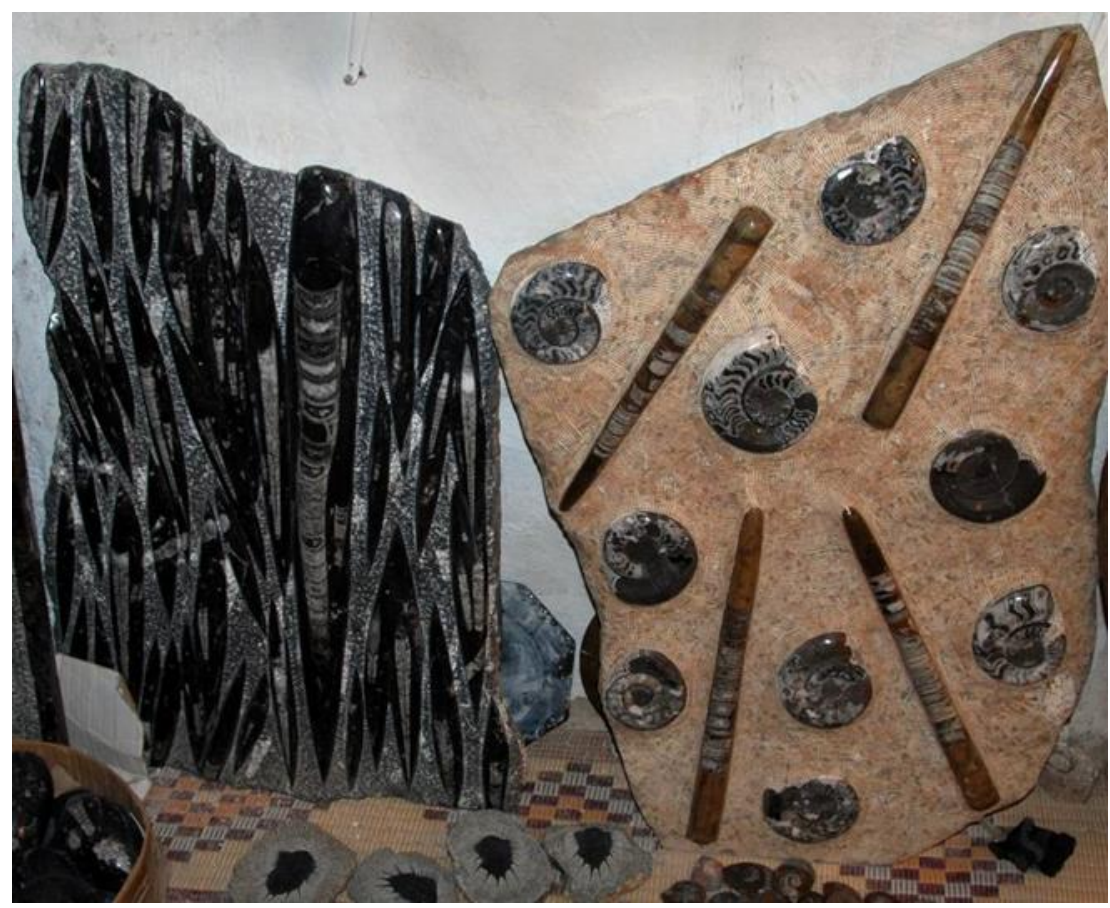

Fig. 5. Limestone slabs rich in Palaeozoic orthocone nautiloids (Siluro-Devonian); South Morocco, Erfoud area. Photo: P. De Wever

Specimens from the latter region, however, are often assembled as composite slabs which include areas simply carved matrix. Geological objects such as this are sold in shops as artwork or ornamental material (and not as a geoheritage objects) and are intended to display beautiful fossils. Most geologists will recognise these pieces as being artificial and some artwork fossil 'designers' do clearly indicate in their shops that they design and make 'artificial' fossils (Fig. 6). When sold, such examples do provide an income for a local community, but the sustainability of the activity and potential danger to the geoheritage of the area is rarely considered.

Fig. 6. The wall of a shop clearly announcing what happens inside: "fabrication des fossiles" [= "fossil manufacturing"] (Erfoud, South Morocco). Photo: P. De Wever

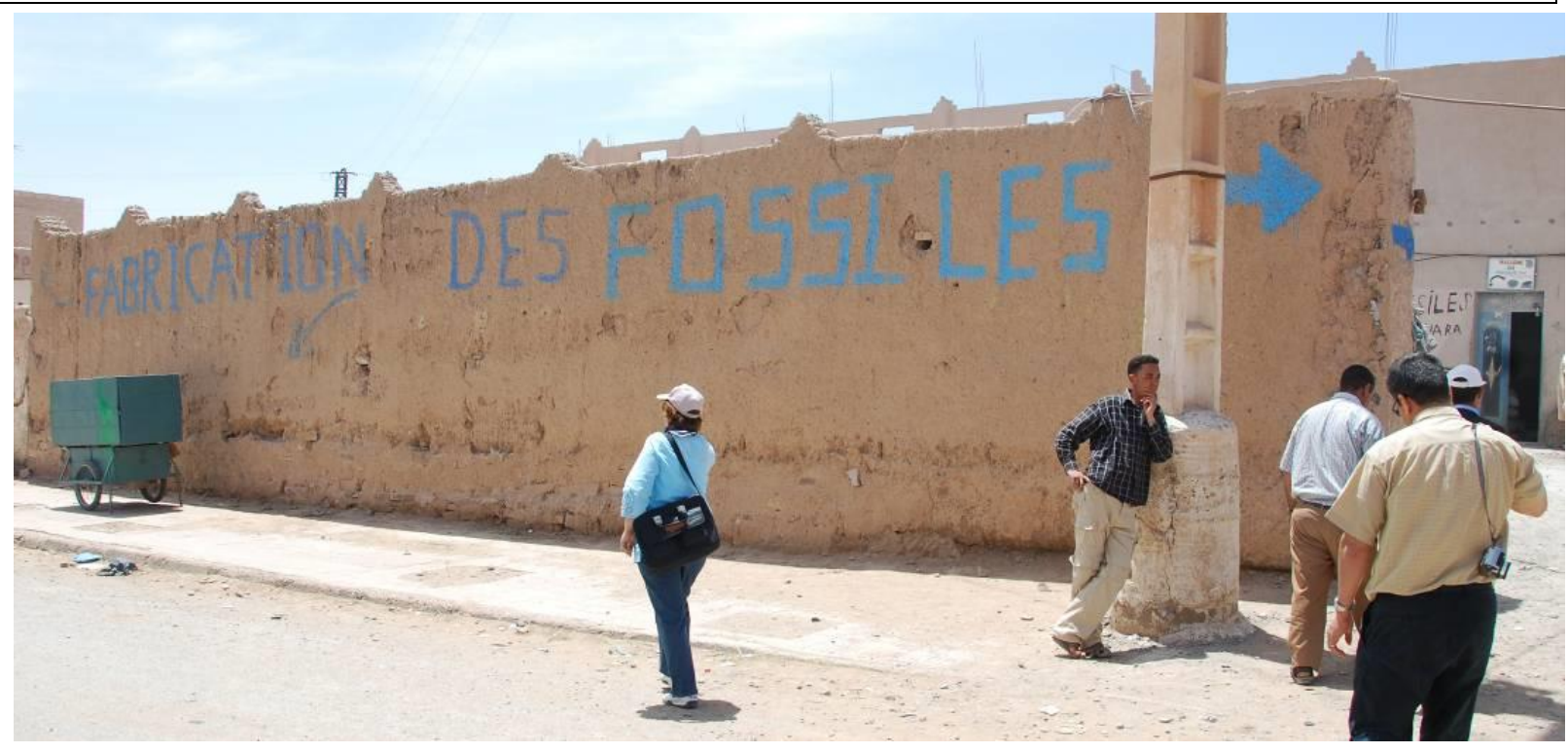


Some objects, by themselves would not belong to heritage, but occasionally gain the status of heritage. By the end of the 20th century, microscopes had become popular and were therefore accessible as personal belongings. Some took this opportunity to create microscopic photographs of tiny objects or micro-organisms such as radiolarians, diatoms and scales of butterfly's wing (Fig. 7). Such images are original and are worthy of being part of our scientific heritage since they testify to a conjunction between the development of technology and private/personal interests and use. The way these microscope slides are mounted is called "lutage à la tourette" which indicates a method of protecting a small object using a thin coil of 'Judean pitch'. The tar is piped onto the slide as it rotates on a small stand, forming a perfect ring. This technique is no longer in practice so this technical testimony adds more value to the image itself.
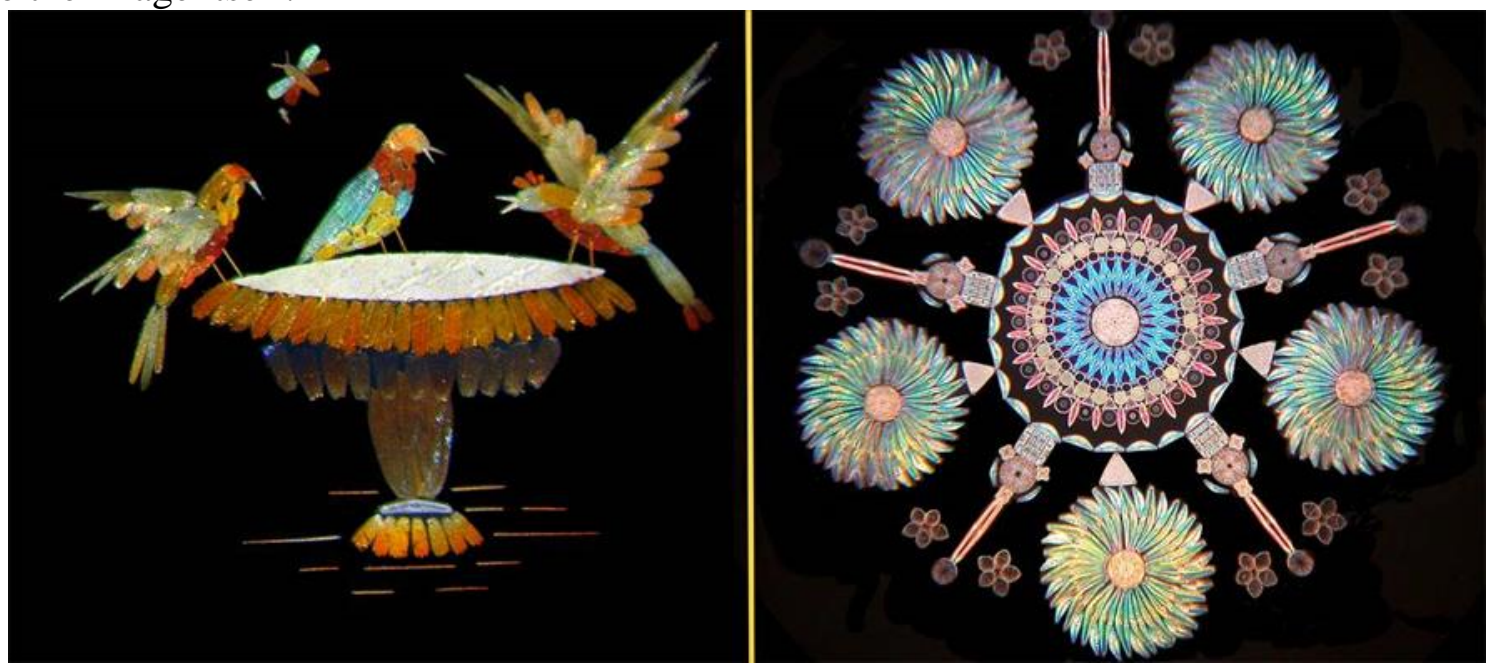

Fig. 7. Two examples of micro-pictures: Lerft: Fountain with birds - a micro-picture made with the scales of butterfly wings (overall size: circa 1 to $2 \mathrm{~mm}$ ). Right: A pink rosette constructed from hundreds of aesthetically arranged diatom shells. Photos: P. Loubry

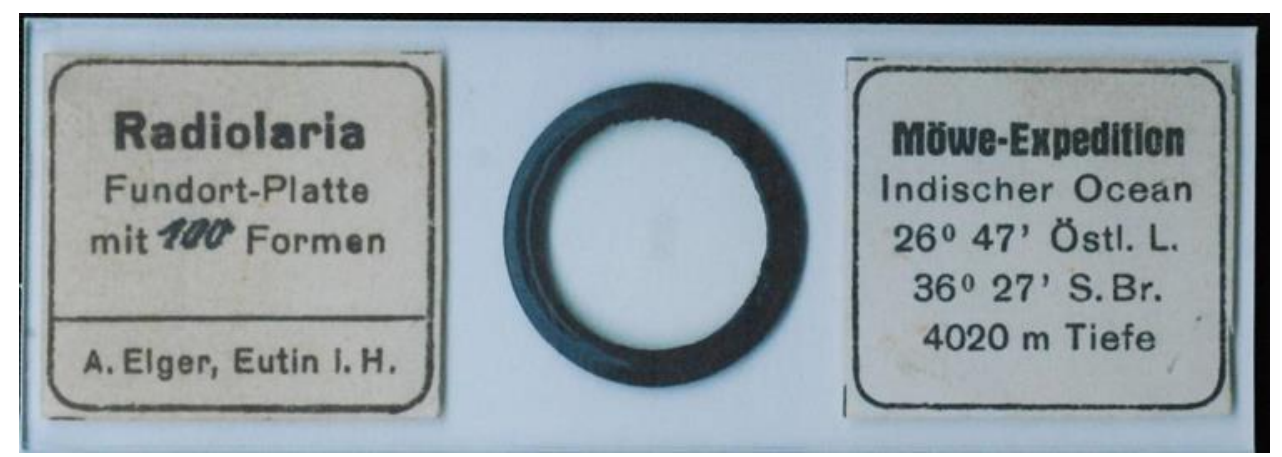

Fig. 8A. Mounted slide with perfectly arranged radiolarians in the middle (grey, almost not visible) with a black coiled bituminous joint protecting them. Photo P. De Wever

Some objects are aesthetic on their own, carry a complementary testimony and hence gain heritage value: for instance, the models of a radiolaria made by German craftsmen as glassworker chef d'oeuvres (Fig. 9). This technique was developed by the German Leopold Blaschka (1822-1895) and later with his son Rudolf (1857-1939). Their work making spectacular glass models of natural history objects began in 1857. Each glass model is a unique blend of art. 


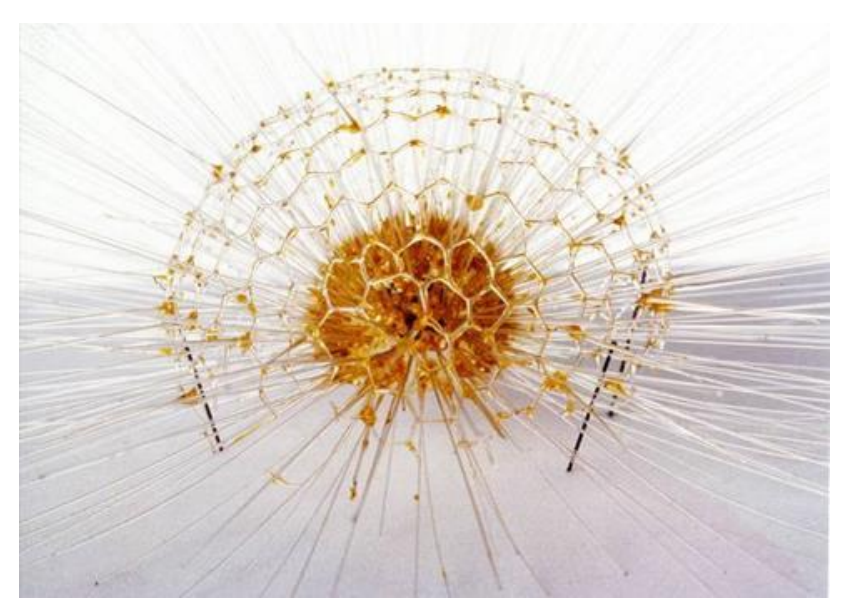

Fig. 9. Model radiolarian approximately $25 \mathrm{~cm}$ in diameter (the original organism is circa 0.2 $\mathrm{mm}$ ). This art object testifies that the craftsman had to observe the original organism under a microscope and then manufacture the thin glassy spines to replicate the fossil structure. Blaschka glass artists.

Some objects have an aesthetic quality even if they do not justify being considered as a particular geoheritage of scientific value, such as the 'Pierre de rêve' (Fig. 10). But, when such a specimen is not isolated but rather belongs to a collection of tens of objects deposited in a museum and moreover when such specimens were gathered by famous individuals such as Roger Caillois (1913-1978), then the collection may in fact belong to a scientifically valued geoheritage. Caillois was well known for his passion for stone and wrote many poems about them (e.g. Pierres in 1966 and L'Écriture des pierres in 1970).

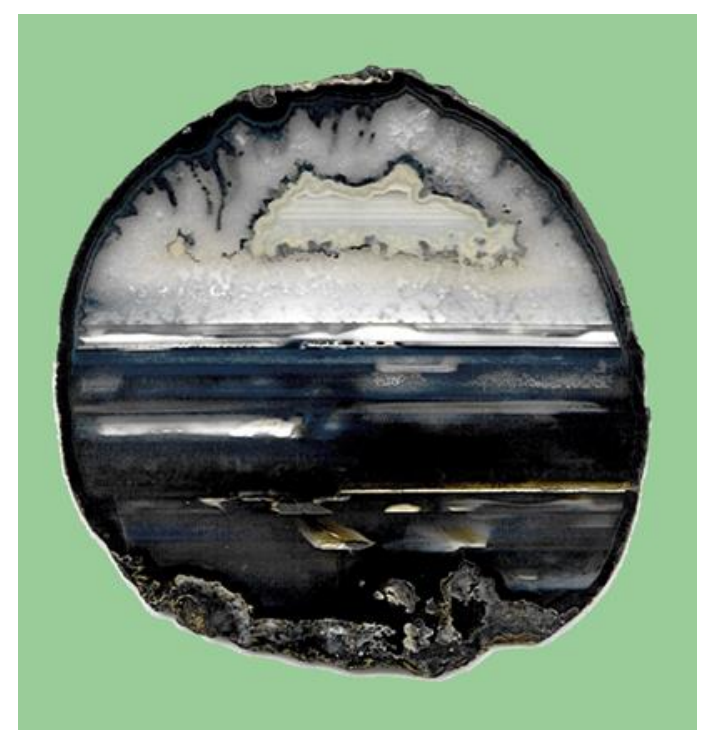

Fig. 10. An example of the Caillois collection of agates known as 'Pierres de rêve' (Dali Shi)

Some landscapes are interesting on their own, although they may not be worth registering on a specific geoheritage list, unless they are are also associated with complementary elements that provide a higher interest level. This is the case for instance for the Sainte-Victoire massif (Provence, France, Fig. 11). This massif corresponds to the front of 
an overthrust. It has been painted by several artists, especially Cezanne, who represented it in more than ten works and who further wrote: "As I told you this morning, I need to know the geology, how Sainte-Victoire is rooting, the geologic colours of its earths, all this touches me, makes me better". In addition, however, Cretaceous red marls on its' western flank are important for dinosaur eggs. The combination of these three elements contributes to an increase in geoheritage value.

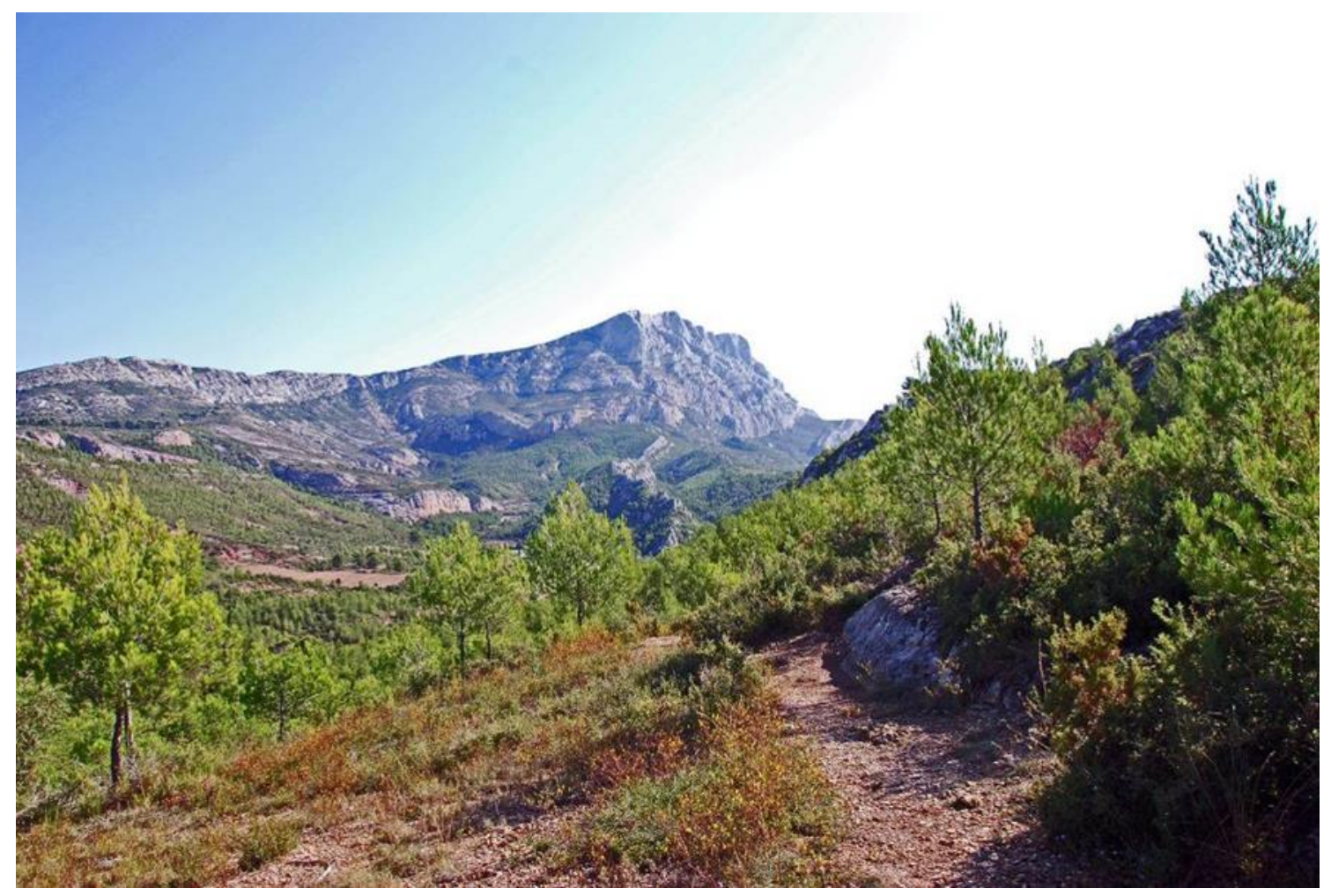

Fig. 11. The Sainte-Victoire massif is the front of a southward overthrust (to the South, on the right). The stratification, almost horizontal on the upper part, is dipping on its front part and becomes almost vertical. This place is also famous because it was frequently painted by Paul Cézanne (Photo: P. De Wever)

A geosite is generally understood to be an exceptional geological place, geographically limited, with one or several geological elements with specific values for scientific, pedagogic, cultural or touristic interest (Brilha et al., 2005). Geosites are usually chosen on the basis of the opinions of experts with a relevant geological knowledge. Geological heritage is an important part of the world's heritage, as it represents the unique record of the evolution of our planet, recorded in a large number of segments. Like a puzzle, these pieces only form a coherent picture when viewed collectively. Unfortunately, only a very limited number of pieces are accessible for human observation.

The term 'geological heritage' is often defined with the notion of 'remarkable geological object' that stands out due to its scientific, educational and historical value, its rareness, its exemplarity, its representativeness, its exceptional state of conservation and its aesthetic quality. Such remarkable geological objects of any size must benefit from in situ or ex situ protection and conservation. Today, protecting heritage is understood as a dynamic process: rather than placing the object beneath a display dome, the conservation approach is a modern, active one, facilitating access to knowledge or research. 


\section{How can we identify the most remarkable sites?}

Geology encompasses a wide variety of topics and its scope in space and time is not always easy to comprehend. If one tries to label sites as 'of international importance', the consideration of priorities has to be fixed. There is no instant method for establishing these priorities, so a systematic approach is, therefore, a necessity. Several sets of data, welldirected and focused judgments are required before any decisions can be taken. It is easy to say that a site is special or unique, but it must also be established in an objectively identified process.

The French Ministry of Environment did not select a specific process framework in the initial phase of the French inventory programme despite the fact that such frameworks were recommended by ProGEO (this general process was, however, used for the Strategy to Create Protected Areas (Stratégie de création d'aires Protégées $=$ SCAP) see section IV).

\section{III- Which geodiversity is there in France?}

The French territory encompasses over $550,000 \mathrm{~km}^{2}$. The total national area, including overseas territory, represents $675,417 \mathrm{~km}^{2}$. The latter territories are located in South America (Guyana), the Atlantic Ocean (Antilles: Guadeloupe, Martinique, Saint-Martin and SaintBarthélemy; Saint-Pierre-et-Miquelon), the Pacific Ocean (French Polynesia, New-Caledonia, Wallis-et-Futuna and Clipperton); the Indian Ocean (La Réunion, Mayotte, Éparses Islands, Crozet islands, Kerguelen Islands and Saint-Paul-et-Amsterdam) and finally in Antarctica (la Terre Adélie).

The geodiversity (from which a geoheritage can be identified and extracted) of a country can be viewed, at a first glance, through a geological map. Detailed topographic maps, therefore, also bear witness of the geological richness by their content and by the patterns of their contours and other mapped features. Herein, we review the relevance and impact of maps in France given their influence on the subsequent geoheritage characterisation of the country.

\section{The first geological maps}

The first map with a global presentation of geology resulted from the desire to establish an inventory of mineral resources of France. The result was a topographical map covered with symbols. This map, signed by Guettard, Lavoisier and Monnet was finished in 1767 and published in 1780 . The 1780 map, however, was preceded by a true geological map with contours drawn by Jean-Etienne Guettard, a naturalist who was supported by the Duc d'Orléans. He presented this first sketch of a map under the name "Carte minéralogique sur la nature du terrein d'une portion de l'Europe" in 1746 (Fig. 12). This map has no true chronological precision, but nevertheless displays the correlation and the continuity of rock bodies on each side of the Channel as it shows an essentially hypothetical continuation of deposits of sandy, marly and shaley beds from the Parisian to the English basins. 


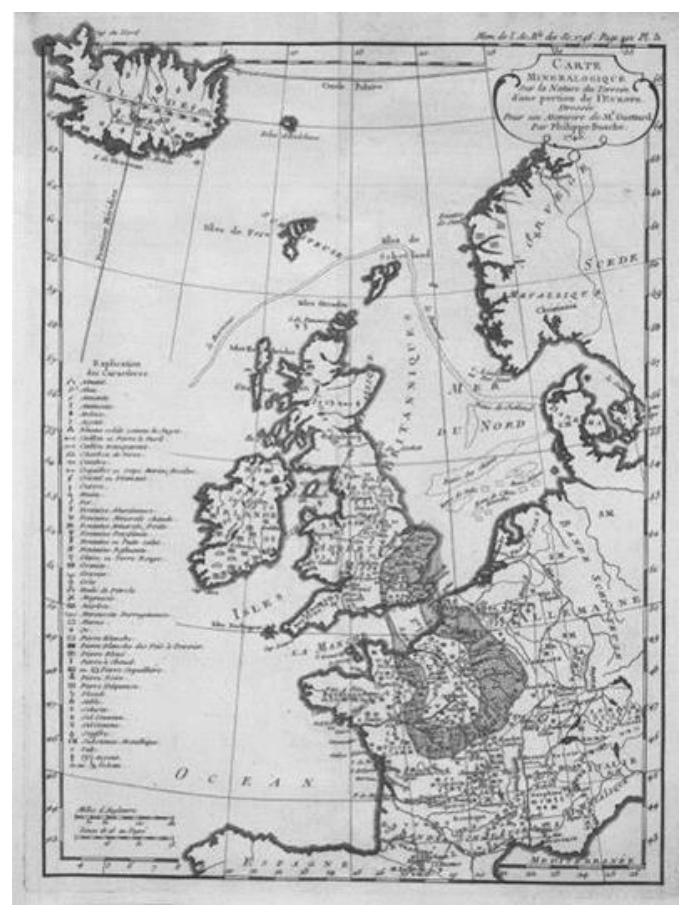

Fig. 12. "Carte minéralogique sur la nature du terrein d'une portion de l'Europe' (JeanEtienne Guettard, 1746). The great novelty of this publication is the hypothetical continuation of strata on each side of the Channel. The author differentiates three main stripes (sandy, marly and shaley) which generally correspond to Cenozoic, Mesozoic and Palaeozoic rocks.

Although William Smith's well known geological map of 1815 of England and Wales was the first national geological map to be published in colour, it "...was neither the first geological map of the 19th Century nor the first to show an ordering of the strata and to make use of the accompanying ordering of their fossil contents. Smith's Paris rivals, Georges Cuvier and Alexandre Brongniart published such a "Geognostique" map of the Paris Basin in 1808, seven years before Smith's map and republished it in 1811 and again in 1822", (Schneer, 1954 - although the 1808 publication did not include a map) (Fig. 13). Later important geological maps of French territories include those published by Jean-Baptiste Julien d'Omalius d'Halloy (Fig. 14) which first coversed fpart of France (1816) and then all France with a part of Belgium, of Germany, of Switzerland and of Italy (1822). Although at the beginning of the nineteenth century, progress in mapping was already represented by contour details andthe use of colour, the concept of a geological map had actually been discussed by Martin Lister in 1684. 


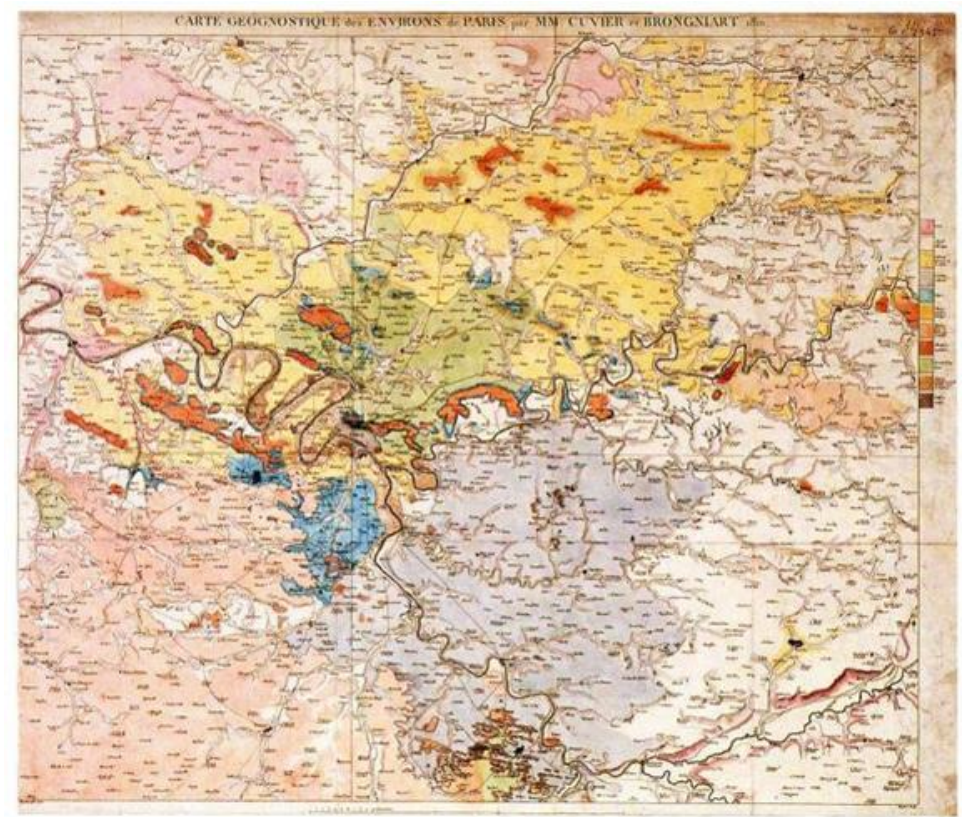

Fig. 13. 'Geognostic' map of the Paris basin, from Cuvier and Brongniart, dated as 1810 and printed in 1811. It covers circa $120 \mathrm{~km}$ (NS) x $150 \mathrm{~km}$ (EW).

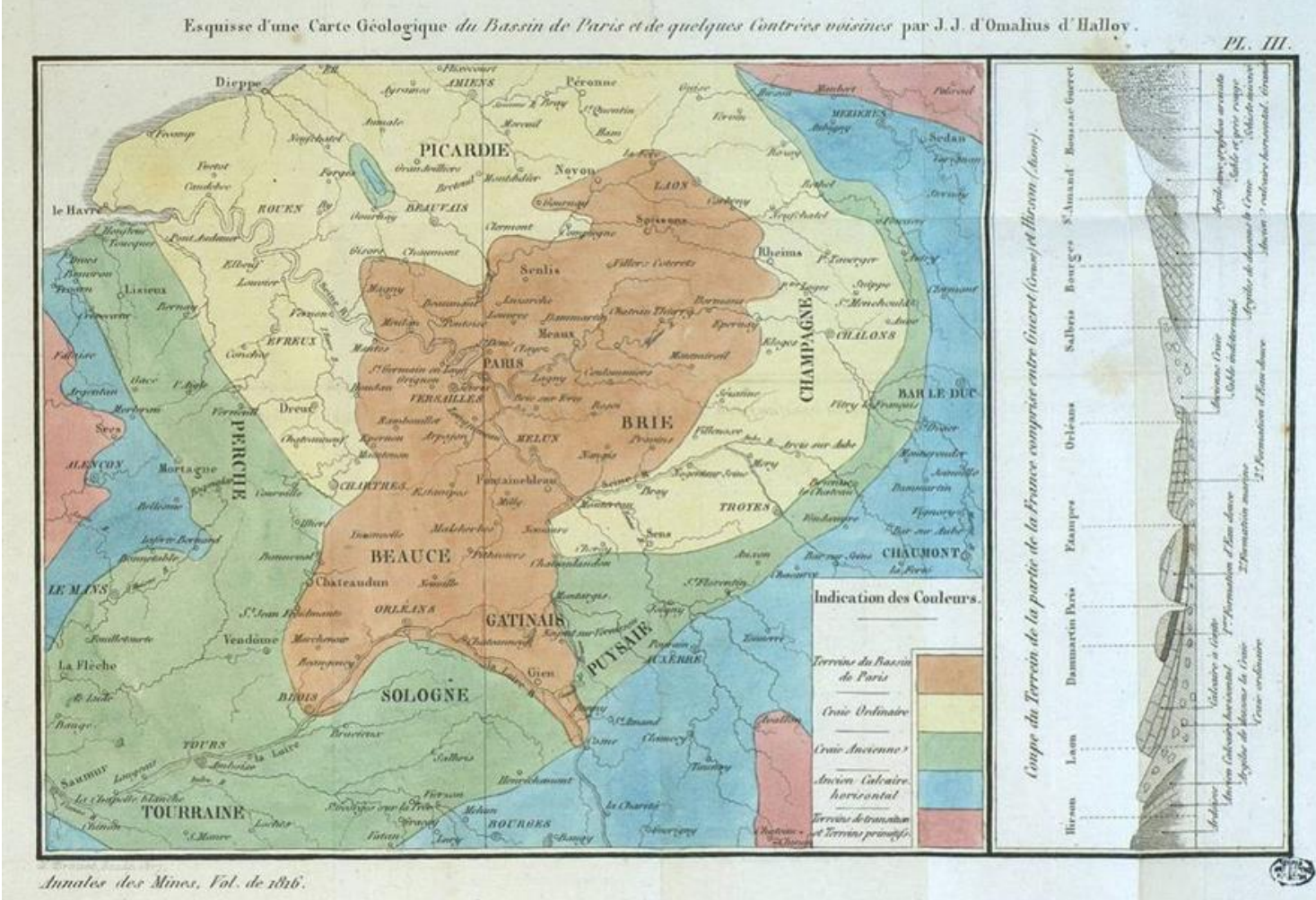

Fig. 14. Geological map of the Paris basin 'Carte géologique du bassin de Paris et de quelques contrées voisines' by d'Omalius d'Halloy (1816). This map demonstrates clearly, for the first time, the famous rings (Jurassic = blue, Cretaceous = green and 'Tertiary' = orange) around the basin. It also displays the first synthetic cross section of this basin with stacked strata (Doc. MNHN Central Library)

Following Guettard's map, it seems that colour appeared on maps in Germany in circa 1770 (with Gläser 1775 and Charpentier 1778). The Belgian Omalius d'Halloy used a set of colours for his map of the Paris basin (1816). On this map he used a pink-red colour for 
basement, blue for the 'old limestone', green for 'old chalk'; pale yellow for the 'ordinary chalk' and orange for 'Parisian terranes' (which corresponds to the Cenozoic'). If we question the reason for these colours, it appears that the decision was likely to have been led by the colours of the rock themselves. Indeed, when limestone cliffs are seen in the countryside they seem to be bluish. At that time the most representative limestone strata were those of the Jura Mountains. These deposits were therefore represented in blue and now represent the Jurassic. In the Paris basin, overlying the Jurassic limestone is a green sand (due to a richness in glauconite) which is quite well known since it supports one of the most important aquifers. These deposits were therefore represented as green and later more generally representing the Cretaceous. More recent sediments in the Paris basin are mainly represented by light sand (white to yellow): hence these colours were used for the Cenozoic. Initially, the colours represented lithology since the first uses of geologic maps were for exploration for natural resources. A homogenization of colours began setting in between 1830 and 1860 with the publication of new maps (Fig. 15 and Fig. 16). Scientific organisations subsequently worked out a consistent scheme of colours to use for maps, including internationally.

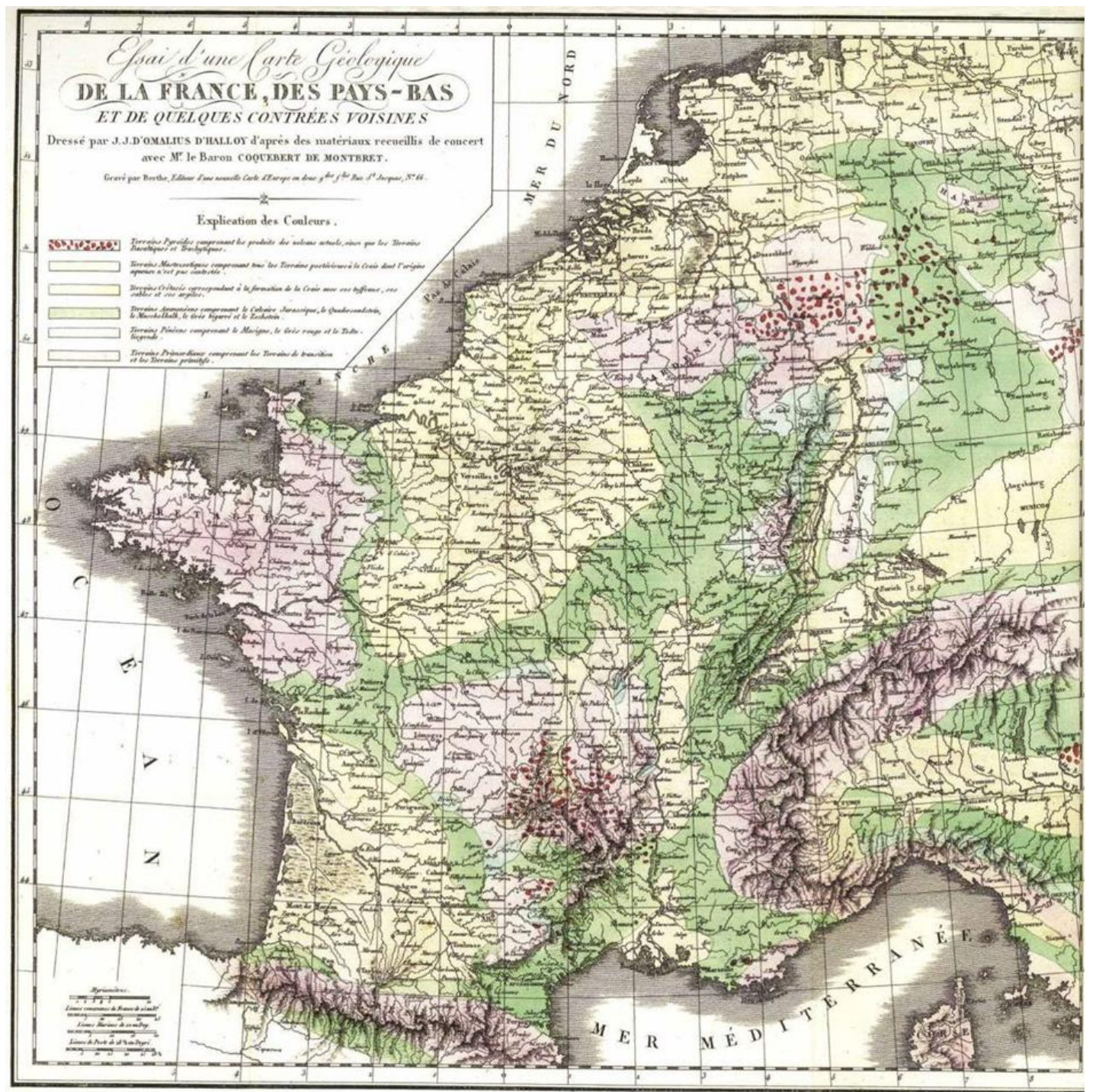

Fig. 15. J.J. d'Omalius d'Halloy's map dated as 1822. It does not appear familiar as a geological map since the colours are not the same as those used today- here for instance the green colour corresponds to the Triassic and Jurassic. 


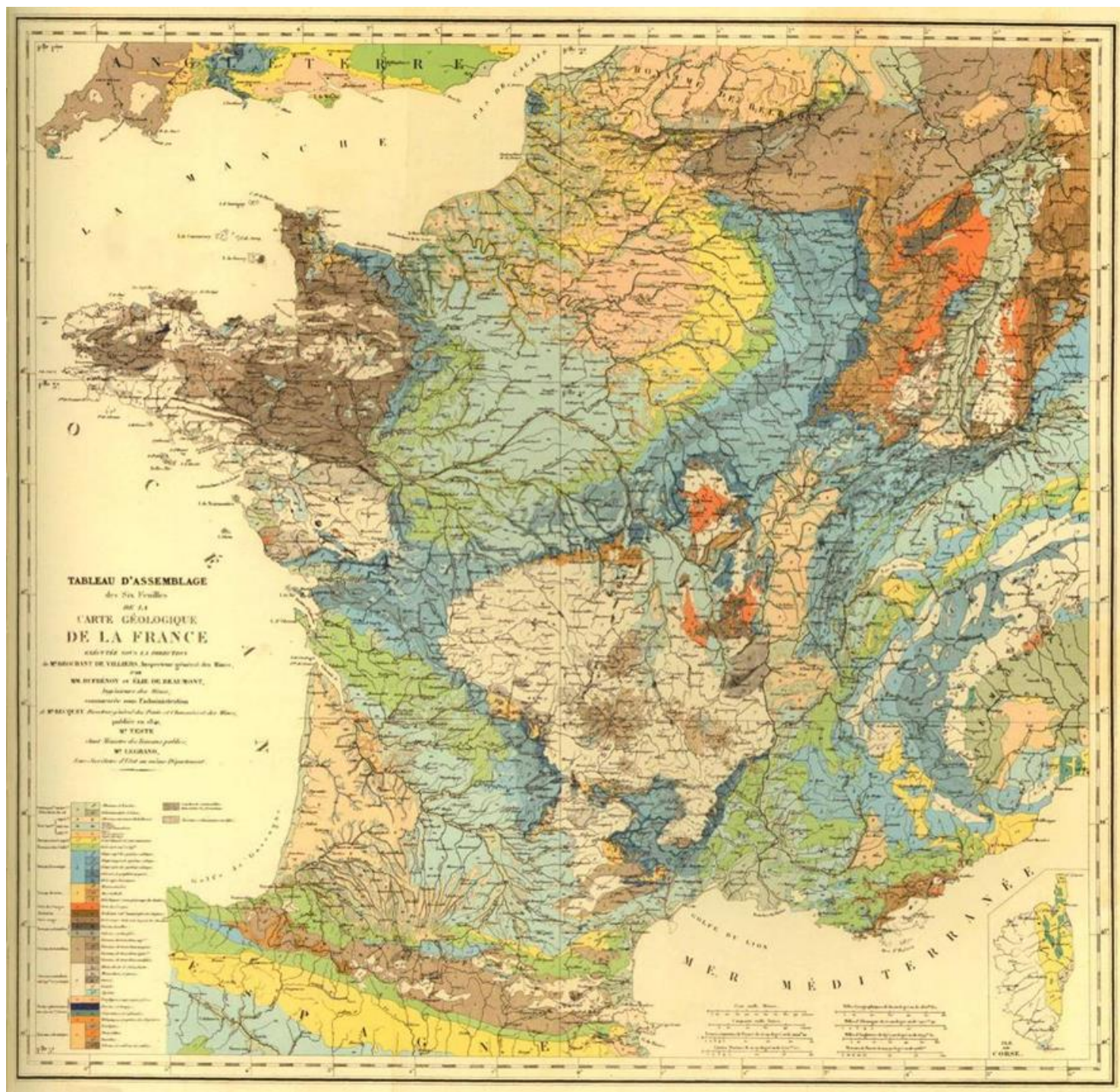

Fig. 16. The Dufrénoy and Elie de Beaumont, dated 1841. This map appears familiar as a geological map since the colours are close to those used today (although the basement is not generally represented by light to dark browns). On this map the subdivisions are henceforth clearly made on age rather than lithology.

On geological maps, colours, therefore can have an international meaning: blue for Jurassic, green for Cretaceous, yellow for Cenozoic, and so on. The history of selection for these colours is not simple, however, butthey remain quite simple in their intent. The first coloured map of the Paris basin (Fig.14) and the one of France (Fig.16) are familiar to our eyes since the colours are close to those used today. On these maps, Jurassic is blue because the limestone displays such a grey-blue tint. Cretaceous is green because of the importance of the Albian glauconite-sands and the Cenozoic is yellow because the sands are often yelloworange coloured. Later, Triassic was represented as pink-violet, because it is characterised by the red bundsandstein, and the Palaeozoic darker as brown. The Carboniferous with its abundant coal, is shown as dark grey. As one can imagine, the acceptance of these colours has been strongly and widely discussed. This was more specifically the case during the second international geological congress in Bologna, in 1881 (Capellini G. 1882). Some countries would have preferred a variation from the three main colours: red, blue and yellow. Others favoured variation on the theme of the colours of the rainbow. Debate was sustained by 
imagination, as well as practical or national reasons but the fact remains that the strength of usage seemed to play an important role.

\section{Some testimonies of the geoheritage in France}

The geodiversity and geoheritage of France is quite remarkable. The main geological objects and sites are well represented: old massifs (Armorican Massif, Ardennes, Massif Central, Saint-Pierre et Miquelon) and more recent folded belts (Alps, Pyrenees, Caledonian Alps), insular arcs (Antilla), hot spots (La Réunion), large igneous Provinces (Kerguelen); large sedimentary basins (Paris Basin, Aquitanian and SE basins), graben basins (Alsace, Limagne). The territory also provides testimony of a history spread over 2 billion years. Geological phenomena such as volcanism (present and past, mid oceanic or intraplate, extensional or compressional), metamorphism, erosion, transport, and sedimentary deposits are well represented. All types of rocks are present (deeply formed, sediments, volcanic, metamorphic, etc.).

There are currently numerous international references on the national territory. Some rocks or minerals keep the name of their origin (Table 1 and 2), or the name of a French geologist/mineralogist (Table 3). This is also true for selected names of the geological time scale (Table 4).

Table 1. Selected rocks (lithotypes) with a name based on a French locality or French geologist.

\begin{tabular}{|c|c|c|}
\hline Rock & Toponym & Remark \\
\hline Ariegite & Ariège department & A variety of pyroxenolite \\
\hline Avezacite & Avezac-Prat, Pyrenees & A variety of hornblendite \\
\hline Cantalite & Verrières, Cantal, Massif Central, Plomb du Cantal & A variety of trachyandesite \\
\hline Corsite & Santa Lucia di Tallano, near Sartène, Corsica & Orbicular gabbro $=$ Napoleonite \\
\hline Domite & Puy-de-Dôme, Massif Central & A variety of trachyte \\
\hline Doreite & Mont-Dore, Massif Central & A variety of trachy-andesite \\
\hline Esterellite & Boulouris, Esterel Massif & A variety of microdiorite \\
\hline Evisite & Evisa, Corsica & A varieties of granite \\
\hline Florinite & Sainte-Florine, Massif Central & A variety of theralite \\
\hline Fraidonite & Fraidon, in Normandy or Cévennes & A variety of kerssantite \\
\hline Kersantite & Kersanton (near Brest), Brittany & $\begin{array}{l}\text { A variety of lamprophyre. Used to } \\
\text { build the famous Breton Calvary. }\end{array}$ \\
\hline Lherzite & Lherz pond, Pyrenees & Hornblendic dykes \\
\hline Lherzolite & Lherz pond, Pyrenees & A variety of peridotite \\
\hline Lindinosite & Lindinosa, Evisa, Corsica & A variety of granite \\
\hline Luscladite & Lusclade, Mont-Dore, Massif Central & A variety of theralite \\
\hline Mareugite & Mareuge, Mont-Dore, Massif Central & A variety of theralite \\
\hline Miagite & Miage glacier, Mont-Blanc, Alps & A variety of orbicular gabbro \\
\hline Napoleonite & Santa Lucia di Tallano, near Sartène, Corsica & Orbicular gabbro $=$ Corsite \\
\hline Oceanite & Piton de la fournaise, La Réunion island & A variety of basalt \\
\hline Ordanchite & La banne d'Ordanche, Massif Central & A variety of theralite \\
\hline Ouenite & Ouen island, New Caledonia & A variety of gabbro \\
\hline Peleite, peléeite & Pelée mountain ; Martinique & A variety of basalt \\
\hline Pyromeride & Mont Vinaigre, Esterel Massif & A variety of rhyolite \\
\hline Sancyite & Puy de Sancy, Massif Central & A variety of trachyandesite \\
\hline Tahitite & Papenoo, Tahiti, Polynesia & A variety of syenite \\
\hline Vaugnerite & Vaugneray (near Lyon) & A variety of syenite \\
\hline Vogesite & Grendelbruch, Vosges mountains & A variety of andesite \\
\hline Vosgesite & Vosges mountains & A variety of lamprophyre \\
\hline Rock & Patronymic & Remark \\
\hline
\end{tabular}




\begin{tabular}{|l|l|l|}
\hline Hauynite, hauynitite & René-Just Haüy (1743-1822), pioneer in crystallography & A variety of basanite rich in haüyne \\
\hline Blavierite & Edouard Blavier & A variety of rhyolite \\
\hline
\end{tabular}

Table 2. Selected minerals with a name based on a French locality.

\begin{tabular}{|c|c|c|}
\hline Mineral & Toponym & Remark \\
\hline Ardennite & Ardennes Mountains & $\begin{array}{l}\text { Occurs in pegmatites and quartz } \\
\text { veins in schist and in magnesium } \\
\text { sediment rich in aluminium }\end{array}$ \\
\hline Autunite & $\begin{array}{l}\text { L'Ouche de Jau, St. Symphorien, and other places near } \\
\text { Autun, Saône-et-Loire, France }\end{array}$ & $\begin{array}{l}\text { Secondary mineral associated with } \\
\text { uraninite }\end{array}$ \\
\hline Bauxite & Baux (or Beaux), near St. Rémy, Bouches-du-Rhône & $\begin{array}{l}\text { Outcomes of granite alteration in } \\
\text { tropical climates }\end{array}$ \\
\hline Bourboulite & $\begin{array}{l}\text { La Bourboule, Mont-Dore Massif, Puy-de-Dôme, } \\
\text { Auvergne }\end{array}$ & \\
\hline Capgaronnite & Cap Garonne Mine & $\begin{array}{l}\text { Occurs in copper-lead mines within } \\
\text { cavities, in Triassic conglomerates } \\
\text { and sandstone }\end{array}$ \\
\hline Carboirite & Carboire, Ariège, Midi-Pyrénées & $\begin{array}{l}\text { Germanium mineral which occurs } \\
\text { in metamorphic zinc deposits of the } \\
\text { French Pyrenees }\end{array}$ \\
\hline Chabournéite & $\begin{array}{l}\text { Chabournéou Glacier, near Jas Roux, Pelvoux Mt., } \\
\text { Valgaudemar, Hautes-Alpes, Provence-Alpes-Côte d'Azur }\end{array}$ & $\begin{array}{l}\text { Jas Roux deposit in dolomitic } \\
\text { limestone, Hautes-Alpes }\end{array}$ \\
\hline Chaméanite & $\begin{array}{l}\text { Chaméane Uranium deposit, Chaméane, Sauxillanges, } \\
\text { Puy-de-Dôme, Auvergne }\end{array}$ & $\begin{array}{l}\text { Late stage deposit in veins cutting } \\
\text { granite }\end{array}$ \\
\hline Chessylite & Chessy-les-Mines (near Lyon) & 'the blue mine' \\
\hline Compreignacite & Margnac-en-Compreignac mine, Haute-Vienne, Limousin & $\begin{array}{l}\text { A very rare oxidation product of } \\
\text { pitchblende in uranium deposits }\end{array}$ \\
\hline Fougèrite & Fougères Forest, Ille-et-Vilaine, Brittany & $\begin{array}{l}\text { (a 'green rust' see also } \\
\text { Trebeurdenite }\end{array}$ \\
\hline Guyanaite & $\begin{array}{l}\text { Merum river, Kamakusa, Mazarumi district, French } \\
\text { Guiana }\end{array}$ & \\
\hline Hureaulite & Village of les Hureaux, Haute Vienne, Limousin & Occurs in pegmatite and granite \\
\hline Ménilite & Ménilmontant, Paris & $\begin{array}{l}\text { A variety of opal, used as a very } \\
\text { specific white pigment by Vincent } \\
\text { Van Gogh }\end{array}$ \\
\hline Montdorite & $\begin{array}{l}\text { Mont-Dore, Charlannes, La Bourboule, Rochefort- } \\
\text { Montagne, Puy-de-Dôme, Auvergne }\end{array}$ & A variety of mica \\
\hline Montebrasite & $\begin{array}{l}\text { Montebras Mine, Montebras-en-Soumans, Boussac, } \\
\text { Creuse, Limousin }\end{array}$ & $\begin{array}{l}\text { Occurs in pegmatites rich in } \\
\text { lithium }\end{array}$ \\
\hline Montmorillonite & Montmorillon, Vienne, Poitou-Charentes & A variety of smectite \\
\hline Nontronite & Nontron, Dordogne, Aquitaine & A variety of smectite \\
\hline Plombièrite & Plombières-les-Bains, Vosges, Lorraine & $\begin{array}{l}\text { A gelatinous substance which } \\
\text { hardens in air, formed from thermal } \\
\text { water }\end{array}$ \\
\hline Ranciéite & Le Rancié Mine, Vicdessos, Ariège, Midi-Pyrénées & Formed in cavities in limonite \\
\hline Romanéchite & Romanèche, Saône-et-Loire & Manganese deposits \\
\hline Rosiéresite & Rosières, Carmaux, Tarn & $\begin{array}{l}\text { Comes from an abandoned copper } \\
\text { mine }\end{array}$ \\
\hline Trébeurderite & Trébeurden, Côte d'Armor & $\begin{array}{l}\text { Nitrate destructive (could be used } \\
\text { to solve the bloom of green algae in } \\
\text { Brittany) }\end{array}$ \\
\hline Trimounsite & Trimouns, municipality of Luzenac, Ariège & Rare earth titanosilicates \\
\hline Vosgite & Cernay, Haut-Rhin, Vosges & An altered plagioclase \\
\hline
\end{tabular}


Table 3. Selected minerals with a name based on a French patronymic.

\begin{tabular}{|c|c|c|}
\hline Mineral & Patronymic & Remarks \\
\hline Adamite & $\begin{array}{l}\text { G.-J. Adam (1795-1881), mineralogist, who supplied the } \\
\text { first specimen }\end{array}$ & $\begin{array}{l}\text { A secondary mineral found in zinc } \\
\text { deposits containing arsenic- } \\
\text { bearing minerals }\end{array}$ \\
\hline Agardite & Jules Agard (1916-2003), geologist, BRGM, Orleans & A rich rare-earth mineral \\
\hline Agriniérite & $\begin{array}{l}\text { Henri Agrinier (1928-1971), engineer in the Mineralogy } \\
\text { Laboratory of the French Atomic Energy Commission }\end{array}$ & $\begin{array}{l}\text { Magnac mine, Compreignac, } \\
\text { Haute-Vienne, Limousin, topotype } \\
\text { in Ariège }\end{array}$ \\
\hline Alluaudite & $\begin{array}{l}\text { François Alluaud (1778-1866), mining engineer of } \\
\text { Limoges, who discovered the mineral }\end{array}$ & $\begin{array}{l}\text { The discovery of the mineral is } \\
\text { disputed: maybe this mineral was } \\
\text { named by Alexis Damour in } \\
\text { honour of François Alluaud }\end{array}$ \\
\hline Asselbornite & $\begin{array}{l}\text { Eric Asselborn (b.1954), mineral collector and surgeon, } \\
\text { Montrevel-en-Bresse, Dijon, in whose collection the } \\
\text { mineral was first found }\end{array}$ & Radioactive mineral \\
\hline Aubertite & $\begin{array}{l}\text { J. Aubert (b.1929), French geophysicist who collected the } \\
\text { first specimens }\end{array}$ & $\begin{array}{l}\text { Comes from an oxidized zone of a } \\
\text { copper deposit in Chile }\end{array}$ \\
\hline Bariandite & Pierre Bariand (b.1933), mineralogist & $\begin{array}{l}\text { Comes from an oxidized zone of } \\
\text { uranium or vanadium deposit }\end{array}$ \\
\hline Barrandite & Joachim Barrande (1799-1883), geologist & Synonym of strengite and variscite \\
\hline Barroisite & Charles Barrois (1851-1939). Geologist, Lille University & A variety of amphibole \\
\hline Beaumontite & Leonce Elie de Beaumont (1798-1874), Geologist & Synonym of heulandite \\
\hline Becquerelite & $\begin{array}{l}\text { Antoine Henri Becquerel (1852-1908), chemist and } \\
\text { physicist who discovered radioactivity in } 1896\end{array}$ & Radioactive mineral \\
\hline Behierite & $\begin{array}{l}\text { Jean Behier (1903-1965), mineralogist, who found the } \\
\text { mineral in } 1959\end{array}$ & Occurs in pegmatite \\
\hline Berthierine & $\begin{array}{l}\text { Pierre Berthier (1782-1861), chemist/mineralogist, who } \\
\text { discovered the bauxite mineral }\end{array}$ & $\begin{array}{l}\text { A variety of serpentine, topotype } \\
\text { in Hayange, Meurthe-et-Moselle }\end{array}$ \\
\hline Berthierite & $\begin{array}{l}\text { Pierre Berthier (1782-1861), chemist and mineralogist, } \\
\text { who discovered the bauxite mineral }\end{array}$ & $\begin{array}{l}\text { Topotype in Chazelles-Haut, } \\
\text { Mercoeur, Haute-Loire, Auvergne }\end{array}$ \\
\hline Bertrandite & Emile Bertrand (1844-1909), mineralogist & $\begin{array}{l}\text { Topotype near the town of Nantes, } \\
\text { France }\end{array}$ \\
\hline Beudantite & $\begin{array}{l}\text { François Sulpice Beudant (1787-1850), mineralogist, } \\
\text { University of Paris }\end{array}$ & $\begin{array}{l}\text { A secondary mineral occurring in } \\
\text { oxidized zones of polymetallic } \\
\text { deposit }\end{array}$ \\
\hline Biotite & $\begin{array}{l}\text { Jean-Baptiste Biot (1774-1862), physicist who studied } \\
\text { the optical properties of the mica }\end{array}$ & $\begin{array}{l}\text { A variety of mica, occurs in } \\
\text { granitic rocks }\end{array}$ \\
\hline Boulangérite & Charles Louis Boulanger (1810-1849), a mining engineer. & $\begin{array}{l}\text { Topotype in Molières, Gard, } \\
\text { Languedoc-Roussillon, easily } \\
\text { confused with Jamesonite }\end{array}$ \\
\hline Bournonite & $\begin{array}{l}\text { Count Jacques Louis de Bournon (1751-1825), } \\
\text { crystallographer and mineralogist. }\end{array}$ & Contains $\mathrm{Pb}, \mathrm{Cu}, \mathrm{Sb}$, and $\mathrm{S}$ \\
\hline Boussingaultite & $\begin{array}{l}\text { Jean-Baptiste Boussingault (1802-1887), chemist, Lyon } \\
\text { University }\end{array}$ & A variety of picromerite \\
\hline Brochantite & $\begin{array}{l}\text { André Jean Marie Brochant de Villiers (1772-1840), } \\
\text { geologist and mineralogist }\end{array}$ & $\begin{array}{l}\text { Secondary mineral, formed in arid } \\
\text { climates or in rapidly oxidizing } \\
\text { copper sulphide deposits }\end{array}$ \\
\hline Carnotite & Marie-Adolphe Carnot (1839-1920), chemist & A radioactive mineral \\
\hline Cassedanneite & $\begin{array}{l}\text { Jacques.P Cassedanne (b.1928), mineralogist, University } \\
\text { of Rio de Janeiro }\end{array}$ & $\begin{array}{l}\text { Occurred on a museum sample } \\
\text { from the oxidized zones of gold- } \\
\text { bearing quartz vein, Russia }\end{array}$ \\
\hline Cesbronite & Fabien Cesbron (1938-), mineralogist, Orléans & $\begin{array}{l}\text { Located in Bambollita mines, } \\
\text { Sonora, Mexico in which two thin } \\
\text { veins are exposed and cesbronite } \\
\text { occurs in only one, in small } \\
\text { quantities (some grams) }\end{array}$ \\
\hline Chenevixite & $\begin{array}{l}\text { Richard Chenevix (1774-1830), chemist, analyst of an } \\
\text { arsenate of copper and iron from Cornwall in } 1801 \text { (later } \\
\text { shown to be Chenevixite). }\end{array}$ & $\begin{array}{l}\text { An uncommon secondary mineral } \\
\text { in the oxidized zone of } \\
\text { hydrothermal polymetallic mineral } \\
\text { deposit }\end{array}$ \\
\hline
\end{tabular}




\begin{tabular}{|c|c|c|}
\hline Chervetite & Jean Chervet (1904-1962), mineralogist. & $\begin{array}{l}\text { Comes from an uranium mine in } \\
\text { Gabon }\end{array}$ \\
\hline Claudetite & $\begin{array}{l}\text { F. Claudet, French chemist, who first described the } \\
\text { natural material. }\end{array}$ & $\begin{array}{l}\text { Produced as a sublimate during } \\
\text { mine fires }\end{array}$ \\
\hline Coquandite & $\begin{array}{l}\text { Henri-Jean-Baptiste Coquand (1813-1881), Professor of } \\
\text { Geology and Mineralogy }\end{array}$ & $\begin{array}{l}\text { This mineral is the third naturally } \\
\text { occurring antimony oxy-sulphate } \\
\text { known }\end{array}$ \\
\hline Cordierite & $\begin{array}{l}\text { Pierre Louis A. Cordier (1777-1861), mining engineer } \\
\text { and geologist, who first studied this species, National } \\
\text { Museum of Natural History, Paris }\end{array}$ & $\begin{array}{l}\text { Occurred in magmatic, } \\
\text { metamorphic and pegmatitic rocks }\end{array}$ \\
\hline Cumengéite & $\begin{array}{l}\text { Edouard Cumenge (1828-1902), a mining engineer who } \\
\text { worked in the Boleo mines, Mexico }\end{array}$ & $\begin{array}{l}\text { It is an extremely rare mineral } \\
\text { which occurs in oxidized zones of } \\
\text { sedimentary copper ores } \\
\text { associated with boleite }\end{array}$ \\
\hline Curienite & $\begin{array}{l}\text { Hubert Curien (1924-2005), mineralogist and } \\
\text { crystallographer }\end{array}$ & $\begin{array}{l}\text { Comes from an uranium and } \\
\text { vanadium mine in Gabon }\end{array}$ \\
\hline Curite & $\begin{array}{l}\text { Pierre Curie (1859-1906), physicist who discovered } \\
\text { radioactivity, radium and polonium with his wife Marie } \\
\text { Curie }\end{array}$ & Radioactive mineral \\
\hline Damourite & A. Damour (1808-1902), mineralogical chemist & A variety of muscovite \\
\hline Daubréite & $\begin{array}{l}\text { Gabriel Auguste Daubrée (1814-1896), mineralogist and } \\
\text { geologist, who worked extensively with meteorites, } \\
\text { National Museum of Natural History, Paris }\end{array}$ & $\begin{array}{l}\text { Occurs in the oxidization zone of a } \\
\text { bismuth deposit, Constancia mine, } \\
\text { Bolivia }\end{array}$ \\
\hline Daubréelite & $\begin{array}{l}\text { Gabriel Auguste Daubrée (1814-1896), mineralogist and } \\
\text { geologist, who worked extensively with meteorites, } \\
\text { National Museum of Natural History, Paris }\end{array}$ & $\begin{array}{l}\text { Found in small amounts in many } \\
\text { meteorites }\end{array}$ \\
\hline Delafossite & $\begin{array}{l}\text { Gabriel Delafosse (1796-1878), mineralogist and } \\
\text { crystallographer }\end{array}$ & $\begin{array}{l}\text { Principally a secondary mineral } \\
\text { which occurred near the base of } \\
\text { the oxidized zone ofcopper } \\
\text { deposits }\end{array}$ \\
\hline Deloryite & $\begin{array}{l}\text { Jean-Claude Delory (b.1953), mineral collector and land } \\
\text { surveyor, who collected the first specimen }\end{array}$ & $\begin{array}{l}\text { Cap Garonne Mine, Le Pradet, } \\
\text { Var, Provence-Alpes-Côte d'Azur }\end{array}$ \\
\hline Dervillite & $\begin{array}{l}\text { Henri Derville, palaeontologist, Strasbourg University, } \\
\text { who noted the original specimen }\end{array}$ & $\begin{array}{l}\text { Found on a museum specimen } \\
\text { from Gabe Gottes Mine, Ste } \\
\text { Marie-aux-Mines, Haut-Rhin, } \\
\text { Alsace }\end{array}$ \\
\hline Descloizite & $\begin{array}{l}\text { Alfred des Cloizeaux (1817-1897), mineralogist who first } \\
\text { described the mineral }\end{array}$ & $\begin{array}{l}\text { A secondary mineral often found } \\
\text { in the oxidation zones of base } \\
\text { metal deposits }\end{array}$ \\
\hline Despujolsite & $\begin{array}{l}\text { Pierre Despujols (b. 1888), founder of the Moroccan } \\
\text { Geologic Survey }\end{array}$ & Hydrothermal manganese deposit \\
\hline Devilline & Henri-Etienne Sainte-Claire Deville (1818-1881), chemist & $\begin{array}{l}\text { Rare and unusual secondary } \\
\text { mineral found in the oxidized } \\
\text { portions of copper sulphide ore } \\
\text { deposit }\end{array}$ \\
\hline Dolomite & $\begin{array}{l}\text { Déodat Gratet de Dolomieu (1750-1801), mineralogist } \\
\text { and geologist, National Museum of Natural History, } \\
\text { Paris. A character, who inspired several authors in } \\
\text { literature (de Laclos ...) }\end{array}$ & $\begin{array}{l}\text { Dolomsite is a rock, andDolomites } \\
\text { are mountain ranges in N. Italy }\end{array}$ \\
\hline Dufrénite & Ours-Pierre- Armand Dufrénoy (1792-1857), mineralogist & Anglar, Haute-Vienne, Limousin \\
\hline Dumortierite & M. Eugène Dumortier (1802-1873), palaeontologist & $\begin{array}{l}\text { Ducare's Quarry, Chaponost, } \\
\text { Beaunant, Rhône-Alpes, used for } \\
\text { the manufacture porcelain }\end{array}$ \\
\hline Dussertite & $\begin{array}{l}\text { Désiré Dussert (1872-1928), mining engineer who } \\
\text { worked in Algeria }\end{array}$ & Product of arsenopyrite alteration \\
\hline Ellenbergerite & $\begin{array}{l}\text { François Ellenberger }(1915-2000) \text {, geologist, founder of } \\
\text { the French Comity for thehistory of geology } \\
\text { (COFRHIGEO) }\end{array}$ & $\begin{array}{l}\text { Occurs as inclusions in pyrope } \\
\text { porphyroblasts }\end{array}$ \\
\hline Faujasite & $\begin{array}{l}\text { Barthélemy Faujas de Saint-Fond (1741-1819), 1st } \\
\text { professor of geology at the National Museum of Natural } \\
\text { History, Paris }\end{array}$ & $\begin{array}{l}\text { It occurs as a rare mineral in } \\
\text { several locations worldwide and is } \\
\text { synthesized industrially }\end{array}$ \\
\hline Fischesserite & $\begin{array}{l}\text { Raymond Fischesser (1911-1991), mineralogist and } \\
\text { crystallographer, former director of the National School } \\
\text { of Mines, Paris }\end{array}$ & No particular remarks \\
\hline Fontanite & François Fontan, mineralogist, University of Toulouse & $\begin{array}{l}\text { A rare secondary mineral from the } \\
\text { Rabejac uranium deposit, near } \\
\text { Lodève, Hérault, France }\end{array}$ \\
\hline
\end{tabular}




\begin{tabular}{|c|c|c|}
\hline Friedelite & Charles Friedel (1832-1899), chemist and mineralogist & $\begin{array}{l}\text { Montagne d'Azet, Adervielle, } \\
\text { Hautes-Pyrénées, France }\end{array}$ \\
\hline Fluckite & $\begin{array}{l}\text { Pierre Fluck, mineralogist at Strasbourg University, who } \\
\text { discovered the first specimen of the species }\end{array}$ & $\begin{array}{l}\text { Gabe-Gottes mine, Saintes-Marie- } \\
\text { aux-Mines, Haut-Rhin, Alsace }\end{array}$ \\
\hline Garniérite & $\begin{array}{l}\text { Jules Garnier (1839-1904), geologist who discovered this } \\
\text { ore }\end{array}$ & $\begin{array}{l}\text { It is a generic name for a green } \\
\text { nickel ore }\end{array}$ \\
\hline Gatelite & $\begin{array}{l}\text { Pierre Gatel, founder president of the « Association } \\
\text { Française de Microminéralogie » (AFM) }\end{array}$ & $\begin{array}{l}\text { Trimouns talc deposit, Luzenac, } \\
\text { Ariege, French Pyrenees }\end{array}$ \\
\hline Gaudefroyite & Abbe Christophe Gaudefroy (1888-1971), mineralogist & $\begin{array}{l}\text { An uncommon hydrothermal } \\
\text { mineral in manganese deposits }\end{array}$ \\
\hline Gaylussite & $\begin{array}{l}\text { Louis Joseph Gay-Lussac (1778-1850), chemist and } \\
\text { physicist }\end{array}$ & $\begin{array}{l}\text { It is an unstable carbonate mineral } \\
\text { which dehydrates in dry air and } \\
\text { decomposes in water }\end{array}$ \\
\hline Geffroyite & $\begin{array}{l}\text { Jacques Geffroy (1918-1993), metallurgist at the French } \\
\text { Atomic Energy Commission }\end{array}$ & $\begin{array}{l}\text { Chaméane Uranium Deposit, } \\
\text { Chaméane, Sauxillanges, Puy-de- } \\
\text { Dôme, Auvergne }\end{array}$ \\
\hline Giraudite & $\begin{array}{l}\text { Roger Giraud, electron microscopy engineer, CNRS, } \\
\text { Orleans }\end{array}$ & $\begin{array}{l}\text { Chaméane Uranium Deposit, } \\
\text { Sauxillanges, Puy-de-Dôme, } \\
\text { Auvergne }\end{array}$ \\
\hline Gonnardite & Ferdinand Gonnard (1833-1923), mineralogist & $\begin{array}{l}\text { La Chaux de Bergonne, Saint- } \\
\text { Germain-Lembron, Puy-de-Dôme, } \\
\text { Auvergne }\end{array}$ \\
\hline Gorceixite & $\begin{array}{l}\text { Claude-Henri Gorceix (1842-1919), mineralogist and } \\
\text { founder of the Mining School in Ouro Preto, Brazil }\end{array}$ & $\begin{array}{l}\text { Secondary mineral, variety of } \\
\text { phosphate }\end{array}$ \\
\hline Grandidierite & $\begin{array}{l}\text { Alfred Grandidier (1836-1921), naturalist and explorer, } \\
\text { an authority in Madagascar }\end{array}$ & $\begin{array}{l}\text { A rare accessory mineral which } \\
\text { occurs in pegmatite, gneisses, } \\
\text { aplites, xenoliths }\end{array}$ \\
\hline Grunerite & $\begin{array}{l}\text { Louis Emmanuel Gruner (1809-1883), Swiss-French } \\
\text { chemist, who first analysed it. }\end{array}$ & $\begin{array}{l}\text { Sarvengude ravine, Collobrières, } \\
\text { Var, Provence-Alpes-Côte d'Azur }\end{array}$ \\
\hline Guarinoite & $\begin{array}{l}\text { Cap Garonne Mine, Le Pradet, Var, France. From a } \\
\text { French collector : André Guarino }\end{array}$ & Belongs to the green rust family \\
\hline Guerinite & $\begin{array}{l}\text { Henri Guerin [1906- ], French chemist who synthesized } \\
\text { the compound. }\end{array}$ & $\begin{array}{l}\text { A recent weathering product in } \\
\text { oxidized arsenic-rich mineral } \\
\text { deposit }\end{array}$ \\
\hline Guettardite & Jean-Etienne Guettard (1715-1786), naturalist & $\begin{array}{l}\text { Low temperature hydrothermal } \\
\text { origin, in marble }\end{array}$ \\
\hline Guilleminite & $\begin{array}{l}\text { Claude Guillemin [1923-1994], chemist and } \\
\text { mineralogist, co-founder of the International } \\
\text { Mineralogical Association. }\end{array}$ & $\begin{array}{l}\text { It is an hydrated selenite of } \\
\text { uranium and barium: it is the first } \\
\text { mineral of natural selenite found }\end{array}$ \\
\hline Haüyne & René Just Haüy (1743-1822), pioneer in crystallography & A variety of feldspathoid \\
\hline Hibonite & Paul Hibon, prospector who discovered this mineral & $\begin{array}{l}\text { Radioactive mineral, present in } \\
\text { meteorites }\end{array}$ \\
\hline Hocartite & $\begin{array}{l}\text { Raymond Hocart (1896-1983), professor of mineralogy at } \\
\text { the University of Paris }\end{array}$ & A variety of stannite \\
\hline Krautite & $\begin{array}{l}\text { François Kraut (1907-1983), mineralogist, National } \\
\text { Museum of Natural History, Paris. He proved the } \\
\text { meteoric origin of the Rochechouart crater. }\end{array}$ & $\begin{array}{l}\text { Found on a mineral specimen from } \\
\text { the Museum which comes from } \\
\text { the famous gold ore of Sacarimb } \\
\text { (Nagyag) in Romania. }\end{array}$ \\
\hline Lacroixite & $\begin{array}{l}\text { After Alfred Lacroix (1863-1948), mineralogist. National } \\
\text { Museum of Natural History, Paris }\end{array}$ & Occurred in druses in granite \\
\hline Laffittite & $\begin{array}{l}\text { Pierre Laffitte (b.1925), director of the National School of } \\
\text { Mines, Paris }\end{array}$ & $\begin{array}{l}\text { Jas Roux, Pelvoux Mt., } \\
\text { Valgaudemar, Hautes-Alpes, } \\
\text { Provence-Alpes-Côte d'Azur } \\
\end{array}$ \\
\hline Laforêtite & $\begin{array}{l}\text { Claude P. Laforêt (b. 1936), metallographer who first } \\
\text { observed the mineral at the Montgros Mine. }\end{array}$ & Pinols, Haute-Loire, Auvergne \\
\hline Laumontite & $\begin{array}{l}\text { Gillet de Laumont (1747-1834), mineralogist who } \\
\text { discovered it }\end{array}$ & $\begin{array}{l}\text { Secondary mineral in basalt and } \\
\text { andesite }\end{array}$ \\
\hline Moissanite & Henri Moissan (1852-1907), chemist & $\begin{array}{l}\text { Encountered in samples of lunar } \\
\text { meteorites }\end{array}$ \\
\hline Morinite & $\begin{array}{l}\text { E.A Morineau, Director of the tin mine at Montebras who } \\
\text { supplied the first specimen }\end{array}$ & $\begin{array}{l}\text { Montebras Mines, Montebras, } \\
\text { Soumans, Creuse, Limousin } \\
\end{array}$ \\
\hline Natrodufrénite & $\begin{array}{l}\text { Ours-Pierre- Armand Dufrenoy (1792-1857), mineralogist } \\
\text { and geologist. Co-author of one of the first geological } \\
\text { maps of France }\end{array}$ & Rochefort-en-Terre, Morbihan \\
\hline Offretite & $\begin{array}{l}\text { Albert Offret (1857-1933), mineralogist and professor of } \\
\text { the faculty of Sciences, Lyon }\end{array}$ & $\begin{array}{l}\text { Mont Simiouse, near Montbrison, } \\
\text { Loire }\end{array}$ \\
\hline Orcelite & Jean Orcel, (1896-1978), mineralogist and professor of & \\
\hline
\end{tabular}




\begin{tabular}{|c|c|c|}
\hline & the National Museum of Natural History, Paris & \\
\hline Parapierrotite & Roland Pierrot (1930-1998), mineralogist & Jas Roux, Hautes-Alpes, \\
\hline Permingeatite & $\begin{array}{l}\text { François Permingeat (1917-1988), mineralogist, } \\
\text { university of Toulouse }\end{array}$ & $\begin{array}{l}\text { A product of hydrothermal } \\
\text { mineralization }\end{array}$ \\
\hline Picotite & $\begin{array}{l}\text { Baron Philippe Picot de Lapeyrouse, mineralogist, } \\
\text { founder of Toulouse Natural History Museum }\end{array}$ & $\begin{array}{l}\text { A dark brown variety of spinel } \\
\text { containing chromium and iron }\end{array}$ \\
\hline pierrotite & mineralogist, Roland Pierrot (1930-1998), mineralogist & $\begin{array}{l}\text { Jas Roux, Pelvoux Mt., } \\
\text { Valgaudemar, Hautes-Alpes }\end{array}$ \\
\hline Pisanite & Félix Pisani (1831-1920), chemist and minerals dealer & A variety of melanterite \\
\hline Proustite & Joseph Louis Proust (1754-1826), chemist & One of the ruby silver ores \\
\hline Rameauite & $\begin{array}{l}\text { Jacques Rameau (1926-1960), French prospector who } \\
\text { discovered the deposit where the mineral was found. }\end{array}$ & $\begin{array}{l}\text { Margnac uranium deposit, } \\
\text { Compreignac, Haute-Vienne, } \\
\text { Limousin }\end{array}$ \\
\hline Roméite & $\begin{array}{l}\text { Jean Baptiste Romé de l'Isle (1736-1790), } \\
\text { eminentcrystallographer }\end{array}$ & $\begin{array}{l}\text { An accessory mineral in } \\
\text { metamorphosed manganese ores }\end{array}$ \\
\hline Roquesite & $\begin{array}{l}\text { Maurice Roques, geologist, university of Clermont- } \\
\text { Ferrand }\end{array}$ & Charrier, Allier, France \\
\hline Roubaultite & $\begin{array}{l}\text { Marcel Roubault (1905-1974), geologist, university of } \\
\text { Nancy. Pioneer and organizer of uranium exploration in } \\
\text { France }\end{array}$ & $\begin{array}{l}\text { Comes from the oxidation zone of } \\
\text { the uranium deposit, Shinkolobwe, } \\
\text { Congo }\end{array}$ \\
\hline Routhierite & $\begin{array}{l}\text { Pierre Routhier (1916-2008), professor of economic } \\
\text { geology }\end{array}$ & $\begin{array}{l}\text { Jas roux, Pelvoux Mt., } \\
\text { Valgaudemar, Hautes-Alpes }\end{array}$ \\
\hline Sabatierite & $\begin{array}{l}\text { Germain Sabatier (b.1923), mineralogist, formerhead of } \\
\text { Orleans's CNRS }\end{array}$ & Formed in calcite veins \\
\hline Sainfeldite & $\begin{array}{l}\text { Paul Sainfeld (b.1916), mineralogist of the Musée de } \\
\text { Minéralogie, Mines School, Paris, who discovered the } \\
\text { mineral }\end{array}$ & $\begin{array}{l}\text { Gabe Gottes mines, Sainte-Marie- } \\
\text { aux-Mines, Haut-Rhin, Alsace }\end{array}$ \\
\hline Schubnelite & $\begin{array}{l}\text { Henri J. Schubnel (b.1935), mineralogist and } \\
\text { gemmologist, National Museum of Natural History, Paris }\end{array}$ & $\begin{array}{l}\text { Discovered at the base of the } \\
\text { oxidized zone of a uranium } \\
\text { deposit, Mounana mine, Gabon }\end{array}$ \\
\hline Sénarmontite & $\begin{array}{l}\text { Henri Hureau de Sénarmont (1808-1862), Mineralogist, } \\
\text { School of Mines, who first described the species. }\end{array}$ & $\begin{array}{l}\text { Comes from oxidation of } \\
\text { antimony minerals }\end{array}$ \\
\hline Thenardite & $\begin{array}{l}\text { Louis JacquesThénard (1777-1857), professor in } \\
\text { chemistry, University of Paris }\end{array}$ & $\begin{array}{l}\text { Non-marine evaporite from arid } \\
\text { climate deposit }\end{array}$ \\
\hline Thérèsemagnanite & $\begin{array}{l}\text { Thérèse Magnan for her contributions to knowledge about } \\
\text { the Cap Garonne mine, Var (France). }\end{array}$ & $\begin{array}{l}\text { From Cap Garonne Mine, Le } \\
\text { Pradet, Var, Provence-Alpes-Côte } \\
\text { d'Azur }\end{array}$ \\
\hline Vauquelinite & $\begin{array}{l}\text { Louis Nicolas Vauquelin (1763-1829), Chemist, } \\
\text { discoverer of chromium. }\end{array}$ & $\begin{array}{l}\text { A rare mineral of the oxidized } \\
\text { zones of hydrothermal base-metal } \\
\text { deposits }\end{array}$ \\
\hline Vesignéite & $\begin{array}{l}\text { Louis Vésigné (1870-1954), mineral collector, former } \\
\text { president of the Mineralogical Society of France }\end{array}$ & $\begin{array}{l}\text { A rare secondary mineral found as } \\
\text { geodes in } \mathrm{Mg} \text { ore in Germany }\end{array}$ \\
\hline Villiaumite & $\begin{array}{l}\text { Maxime Villiaume, traveller who investigated the islands } \\
\text { of Los, former French Guinea, where this mineral was } \\
\text { discovered }\end{array}$ & A variety of halite \\
\hline Weilite & $\begin{array}{l}\text { René Weil (b.1901), professor of mineralogy, University } \\
\text { of Strasbourg, known for his study of Alsatian minerals }\end{array}$ & $\begin{array}{l}\text { Rare arsenate mineral, occurs in } \\
\text { the oxidized zone of the arsenic- } \\
\text { bearing hydrothermal veins }\end{array}$ \\
\hline Wurtzite & Charles A. Wurtz (1817-1884), French chemist & \\
\hline Wyartite & $\begin{array}{l}\text { Jean Wyart (1902-1992), Professor of Mineralogy, } \\
\text { Sorbonne University, Paris }\end{array}$ & A radioactive mineral \\
\hline
\end{tabular}

France possesses more than 40 historical stratotype sites and has a great variety of paleontological and mineralogical sites.

Table 4. Selected French stratotypes. Some of the names are still in use, whereas others are obsolete or have a more local value. Others are currently no longer in use, including Suessonian (d'Orbigny 1852), Parisian (d'Orbigny 1852), Rauracian (Greppin 1867), and Argovian.

* Although the Danian was established primarily in Denmark, sites at Vigny (Val d'Oise) and Laversines (Oise) were defined as co-stratotypes (Desor 1847). 
The colours used are approximately those of the standard French geological time scale.

\begin{tabular}{|c|c|c|}
\hline Stages & Origin & Author \\
\hline RUSCINIAN & Ruscino, Latin name of Perpignan - Pyrénées orientales & Fahlbusch, 1976 \\
\hline REDONIAN & Condate Redonum - Latin name ofRennes-, Ille-et-Vilaine & Dollfus, 1906 \\
\hline BURDIGALIAN & Burdigala [Roman name of Bordeaux], Aquitaine & Depéret, 1892 \\
\hline AQUITANIAN & Aquitaine & Mayer-Eymar, 1858 \\
\hline ASTARACIAN & Astarac, part of the Gers department & Fahlbusch, 1976 \\
\hline ORLEANIAN & Orléans, Loiret & Ginsburg, 1975 \\
\hline AGENIAN & Agen, Lot-et-Garonne & Fahlbusch, 1976 \\
\hline STAMPIAN & Stampae [Latin name ofEtampes], Essonne & d'Orbigny, 1852 \\
\hline SANNOISIAN & Sannois, Val d'Oise & Munier-Chalmas et de Lapparent, 1893 \\
\hline LUDIAN & Ludes, Marne & Munier-Chalmas et de Lapparent, 1893 \\
\hline MARINESIAN & Marines, Val d'Oise & Dollfus, 1907 \\
\hline AUVERSIAN & Auvers-sur-Oise, Val d'Oise & Dollfus, 1880 \\
\hline BIARRITZIAN & Biarritz, Pyrénées-Atlantiques & Hottinger et Schaub, 1960 \\
\hline LUTETIAN & Lutetia [Latin name ofParis] & de Lapparent, 1883 \\
\hline CUISIAN & Cuise-la-Motte, Oise & Dollfus, 1880 \\
\hline SPARNACIAN & Sparnacum [Latin name of. Epernay], Marne & Dollfus, 1880 \\
\hline DANIAN & From Denmark $*$ & Desor, 1846 \\
\hline GARUMNIAN & Garumna [Latin name of. Garonne], Haute-Garonne & Leymerie, 1862 \\
\hline VITROLLIAN & from Vitrolles, Bouches-du-Rhône & Matheron, 1878 \\
\hline ROGNACIAN & Rognac, Bouches-du-Rhône & Villot, 1883 \\
\hline BEGUDIAN & La Bégude [locality], Bouches-du-Rhône & Villot, 1883 \\
\hline FUVELIAN & Fuveau, Bouches-du-Rhône & Matheron, 1878 \\
\hline VALDONNIAN & Valdonne [locality], Bouches-du-Rhône & Matheron, 1878 \\
\hline CAMPANIAN & Champagne, Charente & Coquand, 1857 \\
\hline SANTONIAN & Saintes, Charente-Maritime & Coquand, 1857 \\
\hline CONIACIAN & Cognac, Charente & Coquand, 1857 \\
\hline SENONIAN & Sens, Yonne ; from the Gallic tribes of Sénones & d'Orbigny, 1842 \\
\hline TURONIAN & Tours, Indre-et-Loire & d'Orbigny, 1842 \\
\hline CENOMANIAN & Cenomanum,[Latin name ofLe Mans], Sarthe & d'Orbigny, 1847 \\
\hline ALBIAN & from Alba, Aube river, Aube & d'Orbigny, 1842 \\
\hline CLANSAYESIAN & Clansayes, Drôme & Breitstroffer, 1947 \\
\hline GARGASIAN & Gargas, Vaucluse & Kilian W., 1887 \\
\hline BÉDOULIAN & Bédoule, Bouches-du-Rhône & Toucas, 1888 \\
\hline APTIAN & Apt, Vaucluse & d'Orbigny, 1840 \\
\hline BARREMIAN & Barrême, Alpes-de-Haute-Provence & Coquand, 1862 \\
\hline BERRIASIAN & Berrias, Ardèche & Coquand, 1871 \\
\hline ARDESCIAN & Ardèche [Ardesca] & Toucas, 1890 \\
\hline CRUSSOLIAN & Crussol, Ardèche & Rollier, 1909 \\
\hline SÉQUANIAN & from Séquannes, a Gallic tribe from the source of the Seine & Marcou, 1848 \\
\hline VESULIAN & Vesoul, Haute-Saône & Marcou, 1848 \\
\hline BAJOCIAN & Bayeux, Calvados & d'Orbigny, 1849 \\
\hline TOARCIAN & Thouars, Deux-Sèvres & d'Orbigny, 1849 \\
\hline LOTHARINGIAN & Lorraine ; from Lotharingie, Carolingian province & Haug, 1910 \\
\hline SINEMURIAN & Semur-en-Auxois, Côte-d'Or & d'Orbigny, 1849-1850 \\
\hline HETTANGIAN & Hettange-Grande, Moselle & Renevier, 1864 \\
\hline AUTUNIAN & Autun, Saône-et-Loire & Bergeron, 1889 \\
\hline STEPHANIAN & \begin{tabular}{|l|} 
Saint-Etienne, Loire \\
\end{tabular} & Munier-Chalmas et de Lapparent, 1893 \\
\hline STRUNIAN & Etroeungt, Nord & Barrois, 1913 \\
\hline GIVETIAN & Givet, Ardennes & Gosselet, 1879 \\
\hline BRIOVERIAN & Brioveria, ancient Celtic name of Saint-Lô, Manche & Barrois, 1899 \\
\hline PENTEVRIAN & Penthièvre, Saint-Brieuc bay & Cogné, 1959 \\
\hline
\end{tabular}


Other stratotypes reflect a combination between science and culture (based on a lithic industry) because they were established for prehistoric archaeology. They are listed here because of this link (Table 5).

Table 5. Selected Prehistoric stages with a name based on a French patronym.

\begin{tabular}{|c|c|c|}
\hline Stages & Origin & Author \\
\hline Abbevillian & Abbeville (Somme) & Boucher de Perthes, 1836. \\
\hline Acheulean & Saint-Acheul near Amiens (Somme) & Gabriel de Mortillet, 1872 \\
\hline Artenacian & Artenac (Charente) & ? \\
\hline Aurignacian & Aurignac cave, Haute-Garonne & Henri Breuil \& Émile Cartailhac, 1906 \\
\hline $\begin{array}{c}\text { Azilian } \\
(=\text { Tourassian })\end{array}$ & $\begin{array}{c}\text { Mas d'Azil cave, Ariège } \\
\text { (La Tourasse cave, Saint-Martory; Haute-Garonne) }\end{array}$ & $\begin{array}{c}\text { Edouard Piette, } 1889 \text { (Gabriel de } \\
\text { Mortillet in1872) }\end{array}$ \\
\hline Badegoulian & Badegoule, Dordogne & André Cheynier, 1938, Vignard, 1965? \\
\hline Castelnovian & Châteauneuf-les-Martigues (Bouches-du-Rhône) & Max Escalon de Fonton, 1956 \\
\hline Chassean & Chassey-le-Camp (Saône-et-Loire) & J. Déchelette, 1912 \\
\hline $\begin{array}{c}\text { Chatelperronian } \\
\text { (= Castelperonnien) }\end{array}$ & Châtelperron & Henri Breuil, 1906 \\
\hline Chellean & Chelles (Seine-et-Marne) & Gabriel de Mortillet, 1878 \\
\hline Gravettian & La Gravette shelter, near Bayrac, Dordogne & Fernad Lacorre, 1960 \\
\hline Levalloisian & Levallois-Perret quarries (Hauts-de-Seine) & Victore Commont? \\
\hline Magdalenian & La Madeleine near Tursac, Dordogne (Upper Palaeolithic) & Gabriel de Mortillet, 1883 \\
\hline Montadian & La Montade cave, Plan-de-Cuques (Bouches-du-Rhône) & Max Escalon de Fonton, 1954 \\
\hline Mousterian & Moustier shelter, Peyzac-le-Moustier (Dordogne), & Édouard Lartet, 1860 \\
\hline Peu Richardian & Peu Richard hill, Thénac (Charente-Maritime) & M. Colle, 1956 \\
\hline Sauveterrian & Sauveterre-le-Lémance, Lot-et-Garonne & Laurent Coulonges, 1928 \\
\hline Solutrean & La Roche de Solutré (Saône-et-Loire) & Henry Testot-Ferry, 1866 \\
\hline Thenacian & Thénac (Charente-Maritime) & $?$ \\
\hline
\end{tabular}

Apart from a richness of in situ elements present at geoheritage sites, museums and universities host millions of objects (i.e. an ex situ geoheritage) represented by rocks, fossils, minerals, piston and drilling cores and a suite of other items often with the associated documentation.

The protection of geoheritage must necessarily rely on a legal status specifically for remarkable geological objects. The so-called French 'Barnier' law passed in 1995, which was intended to establish a national listing of protected geological sites, but this never saw fruition. Such a list, however, is not easy to compile and it requires an inventory and an evaluation. Such an inventory is required by law, which in France was enacted in 2002. In April 2007, the French ministry in charge of the environment launched the inventory of the nation's geological heritage. A national methodology was developed and a dedicated software program was produced and widely distributed (Fig. 18). 


\section{IV - Inventory}

\section{Why and how?}

The law enacted in 2002 grants formal recognition to the notion of geological heritage for the first time (French Law 2002-276, February $27^{\text {th }}$, art.411-5, see annexe 1).

"The inventory of natural heritage is set up for the entire national territory of France. A natural inventory encompasses the inventory of the richness of ecologic, faunistic, floristic, geologic, mineralogical and paleontological richness ${ }^{l}$. It is also defines that the inventory is conducted under the scientific responsibility of the National Museum of Natural History of France".

To properly conduct the inventory, the methodology must be fixed at a national scale by the ministry in charge of the environment in order to maintain homogeneity at the national level. The process of documentation for the inventory is 'bottom-up', however. This methodology has now been fixed for the national territory (both in continental and overseas areas) but the data are documented at a regional scale and discussed by a specific commission composed of geologists (professional or amateur) from academic, industrial or education disciplines. This commission represents a regional committee for geoheritage (Commission régionale pour le patrimoine géologique or CRPG) and each committee has about a dozen members.

The collection of data is then discussed at a regional level by Départments or for the entire Région. The list of sites that the CRPG agrees upon is submitted to the regional committee (Conseil scientifique régional du patrimoine naturel, CSRPN of figure 17). The data are combined into a database with online access (iGeotope), homogenised, checked by a regional commission, then transferred to the National Museum of Natural History where it is examined by a national commission composed of geologists from different disciplines, different regions, and belonging to different institutions, and appointed by the National Museum of Natural History.

The ratified site data are stored at the national level and transferred onto a public website: http://inpn.mnhn.fr ${ }^{2}$ for widespread public use. These data are on the same website as other scientific inventories dealing with nature. The advantage for this geoheritage data collection process is that it is perfectly compatible with other data sets for nature (flora, fauna, ecosystems, habitats $-\mathrm{ZNIEFF}^{3}$, etc.).

\footnotetext{
${ }^{1}$ The terms «mineralogical and paleontological» are superfluous since these disciplines are subdivisions of geology but since they are specifically mentioned in the law, we let them here

${ }^{2}$ INPN = Inventaire national du patrimoine naturel (Natural heritage of France website)

${ }^{3}$ ZNIEFF : Zone Naturelle d'Intérêt Écologique, Floristique et Faunistique = Natural zone of ecologic, faunistic or floristic interest.
} 


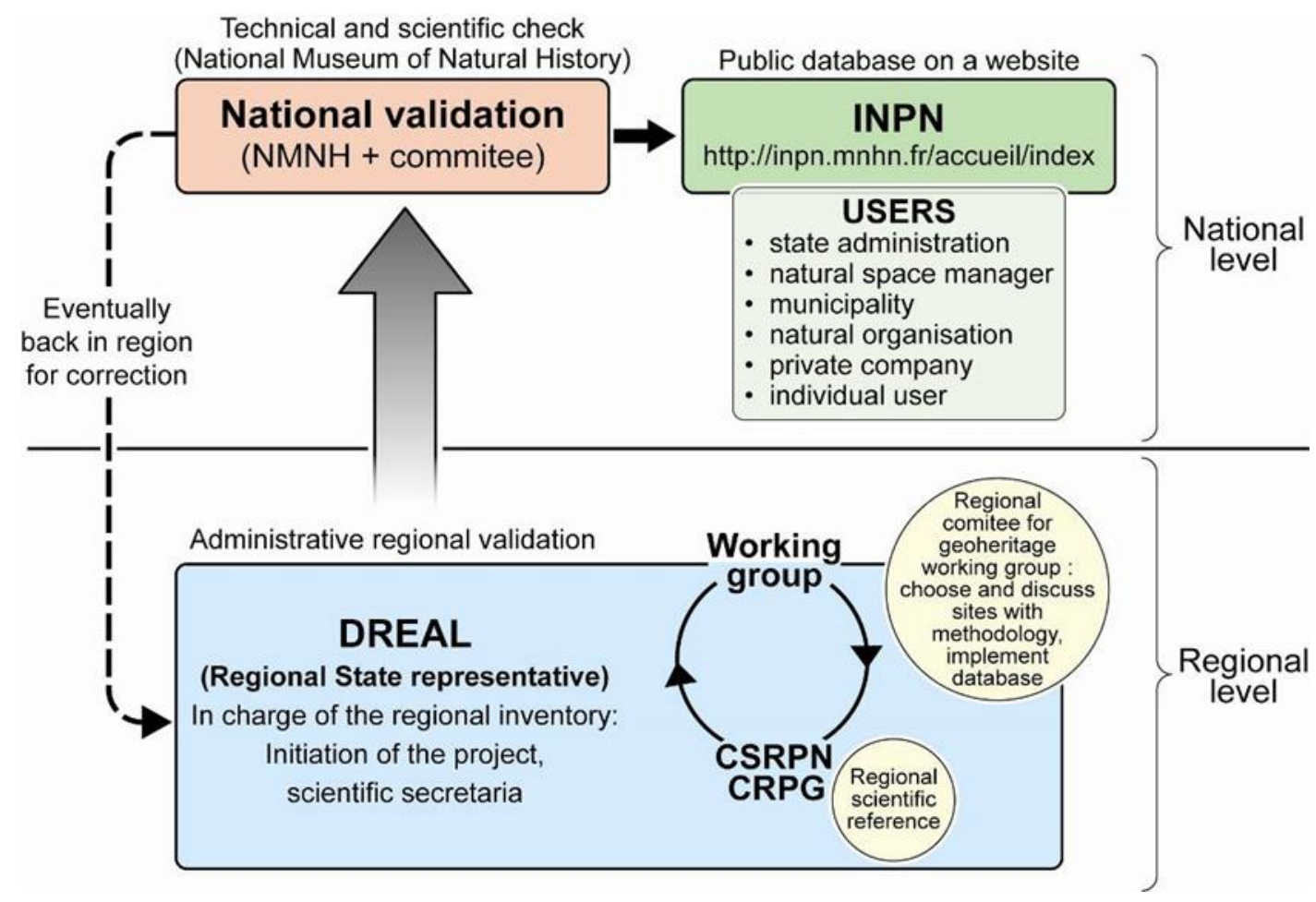

Fig. 17. Flow chart of the protocol for geoheritage inventory.

The inventory of geological sites is a result of two main activities: evaluating the most important sites, then establishing their organisation into a hierarchy. It is only at this second stage of the process that the most remarkable sites are identified and recorded on a national list. The geoheritage site can be selected according to the number of calculated 'stars' (Table 6). This inventory also includes collections in museums or in universities.

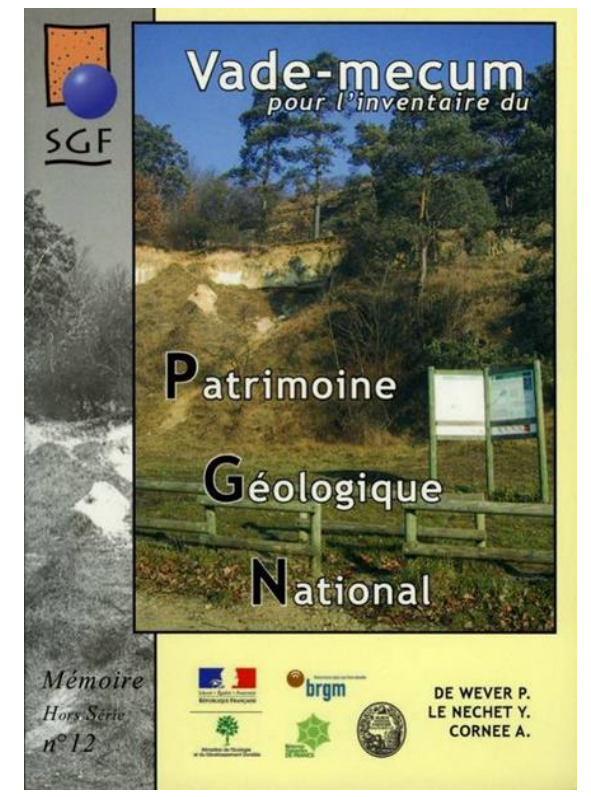

Fig. 18. The methodology for the inventory of national geoheritage is presented in a dedicated volume of the Société géologique de France (De Wever et al., 2006). 
The CRPG experts also provide a bibliographic set of references which are required, such as geological maps, journal articles, handbooks, geological maps, and so on. The inventory characterisation and the assessment of geological heritage are carried out in a systematic manner. Several local or regional inventories which already existed at an administrative department level or carried out by a geoconservation organisation (Réserves Naturelles de France, Parcs Naturels, etc) have been incorporated in the national procedure.

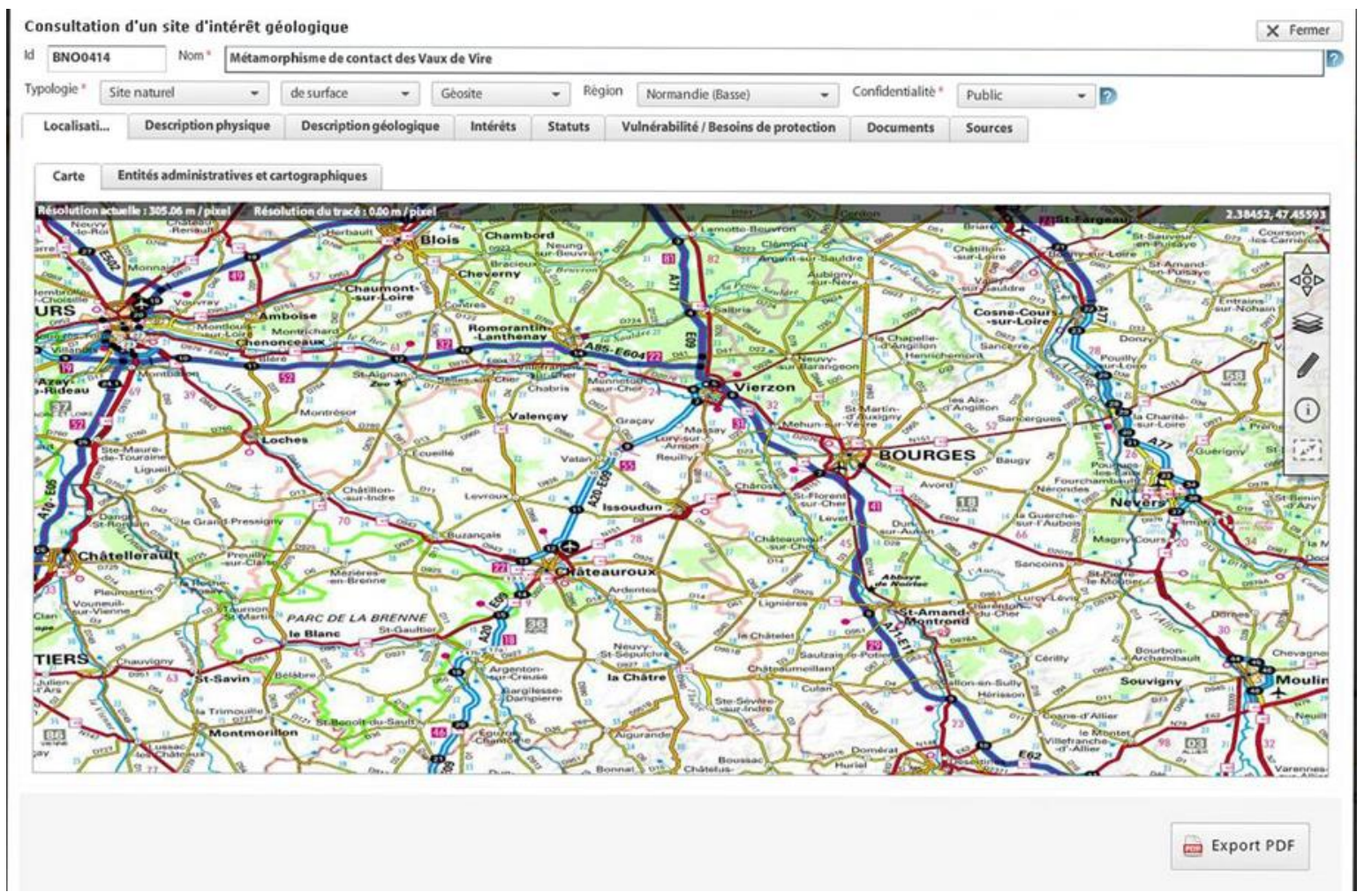

Fig. 19. Screen-shot from the website 'iGéotope'. Users can gain access through this interface through a passcode and can directly access the database.

\section{The content of each file of the inventory:}

For each site, it is mandatory that a certain number of items are included (Fig. 19 and Appendix 2):

- A site identifier (automatically provided by the computer) to avoid duplication of site numbers. The identification code is composed of an acronym of the region's name $+\mathrm{a}$ number. For example (based on figure 19): BNO0414 for the "Basse-NOrmandie" region.

- A name (usually a locality name), with the type of rock and age; e.g., 'Metamorphisme de contact des Vaux de Vire = Vaux de Vire (locality name) contact metamorphism.

- A typology: such as 'geosite de surface' (surface geosite) or 'natural/anthropic'.

- An indication of confidentiality: this field defines if the data may be publicised or should remain confidential (accessible only on request). For example, in this case it is 'Public".

- A location with: 
$\bigcirc$ at least one geographic coordinate (several polygons can be included per site), in a GIS (geographic information system) format so that they can be mapped and integrated with other natural data (fauna, flora, etc.) (Fig. 20).

0 at least one reference to a topographic map (from the national geographic Institute). For example, VIRE (1414E) and SAINT-SEVER-CALVADOS (1414O). Several topographic maps may be referenced.

o at least one reference to a geological map (name and number of sheet). For example 'VIRE 0174', which is the name and number of the geological map where the site is located. Several geological maps may be referenced.

- a specific region. This inventory program is constructed by the regional state representative, so sites can only be in one region. A "trans-regional" site cannot be processed as is. Instead, the part on each side needs to be processed separately by the respective administration.

$\circ$ at least one department (101 departments for the whole territory). For example, in this case it is 'Calvados'. Several departments may be referenced.

$\bigcirc$ at least one municipality. For example, in this case, it is 'Vire'. Several municipalities may be referenced.

○ a surface (even if only approximate) in hectares or square kilometres, 3.6 hectares in this example.

- A physical description of the site.

- A geological description of the site:

- full description of the different elements and phenomena.

- at least one geological age, both stratigraphic and numerical (for example, in this example: Brioverian, $540 \mathrm{Ma}$ ).

- a GILGES code (The Global Indicative List of GEological Sites) is an international standard of classification. For example here 'D category': sedimentary petrology, metamorphic, igneous, texture and structure.

- A main geological interest, 'Metamorphism' in this case.

- A secondary geological interest, 'Plutonism, in this case. A site could have no secondary interest.

- An indication of rarity (scale or level of significance), which is "regional"in this case.

- An evaluation of patrimonial interest: e.g. 28 (on a scale of 48).

- A rating about the need of protection: e.g. 5 (on a scale of 12).

- Bibliographic references.

- Graphic documents (photo, map, cross-section, etc.).

- Names of one or several authors.

(See annex 2 for the whole example of a geological site.) 

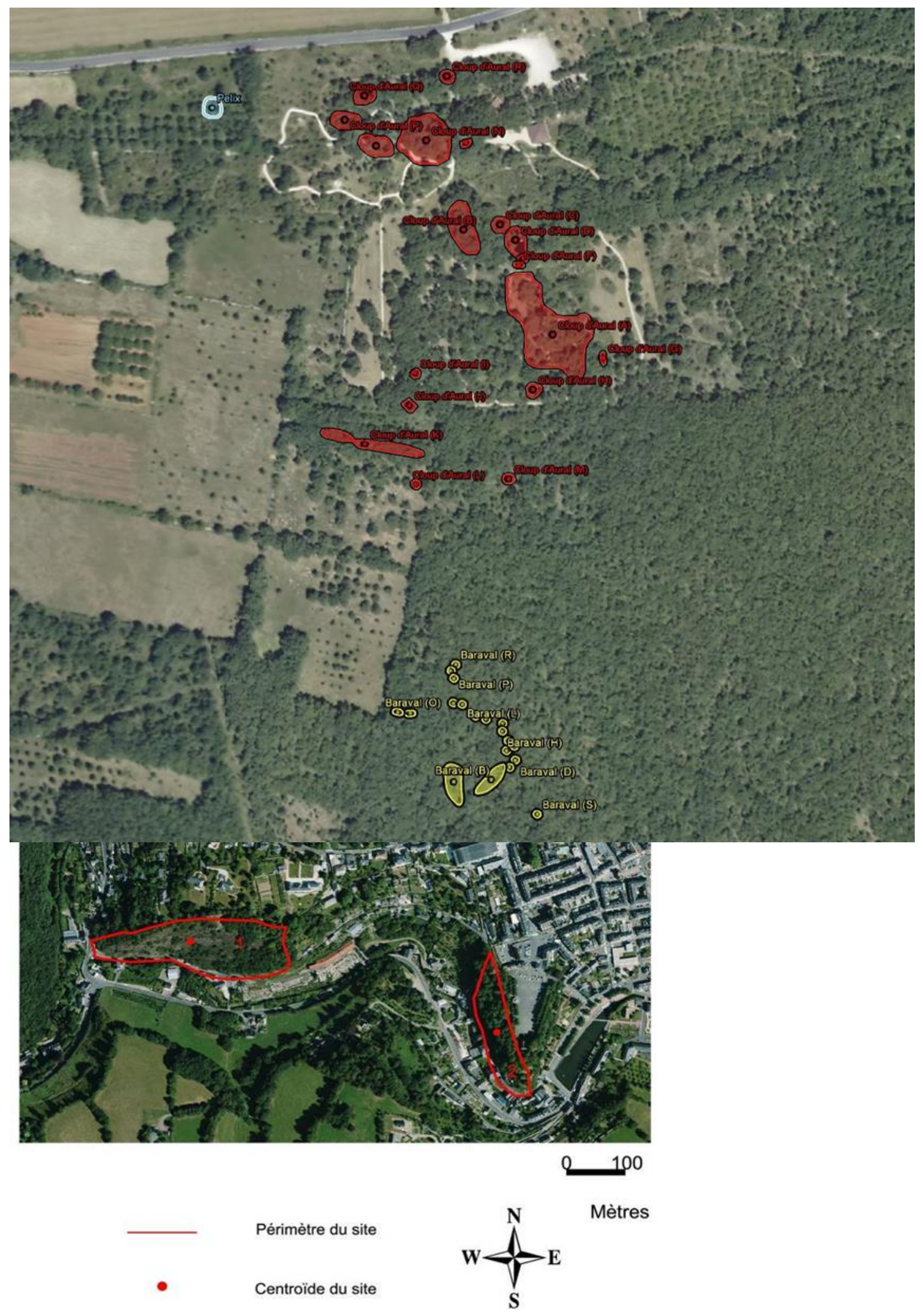

Fig. 20. Two examples of polygons for a site. A - In Normandy (NW France)

B-Example of polygons and points used for the location of three geological sites. Phosphorites du Quercy Area. Bach, Lot (S France). 
For each site, several scores are assigned and a coefficient is also attributed which is related to the relative importance of the topic (Table 6). The scores from 0 to 3 are related to the importance of the site, i.e. its value.

Table 6. Criteria used to calculate the patrimonial interest

\begin{tabular}{|c|c|c|}
\hline Criterion & Value: from 0 to 3 & coefficient \\
\hline Main geological interest & $\begin{array}{c}\text { From weak (0) interest } \\
\text { to remarkable (3) }\end{array}$ & 4 \\
\hline Secondary geological interest & $\begin{array}{c}\text { From no interest (0) } \\
\text { to remarkable (3) }\end{array}$ & 3 \\
\hline Educational interest & $\begin{array}{c}\text { From no interest (0) } \\
\text { to remarkable (3) }\end{array}$ & 2 \\
\hline Historical interest & $\begin{array}{c}\text { From no interest (0) } \\
\text { to remarkable (3) }\end{array}$ & 2 \\
\hline Rarity of site & From common(0)to rare (3) & 2 \\
\hline Preservation state & From poorly (0) to well preserved (3) & \\
\hline
\end{tabular}

In this example, the global note varies from 4 to 48 .

According to the note obtained on the patrimonial interest, a number of stars are attributed:

Note $\leq 10$ :

Note from 11 to 20:

no star

Note from 21 to 30:

$*$

Note from 31 to 48:

**

$* * *$

These stars categorise the importance of the sites within France - national importance is established through comparisons with all similar sites nationally and international significance though a similar international comparison (although these latter assessments are not the primary aim of the initial national process).

In addition to this information, it is also useful to define the need for protection to avoid destruction if a site is under natural or anthropic threat (Table 7). Therefore, a level of protection is calculated for each site on the following basis:

Table 7 Criteria used to define the need for site protection

\begin{tabular}{|l|l|}
\hline Criterion & Level, from 0 to 3 \\
\hline Geoheritage interest & According to the number of stars \\
\hline Natural vulnerability & From none to extreme threat \\
\hline Anthropic threat & From none to extreme threat \\
\hline Effective protection & From maximum to no protection. \\
\hline
\end{tabular}

The level establishes the need for protection and varies between 0 (no threat) to 12 (absolutely necessary to protect).The double level of examination (regional committee and national committee) allows a homogenization at a regional and national level. 
This inventory is a open in order that geosites can be added, modified or deleted from the list at any time. For this reason, some regions were able to establish a list with only a few sites whereas other regions chose to have as many geosites as possible from the beginning of the process. For example, the Nord-Pas-de-Calais Region fixed only circa 60 sites whereas the Midi-Pyrénées Region is working on more than 1,200 sites.

\section{Status in autumn 2014}

In mid-2011, the Ministry in charge of the inventory launched a general inquiry on the progress of the inventory project within the different regions. The survey has been conducted continuously since that date. The Ministry sent a questionnaire to its contacts in the regions (Directions Régionales de l'Environnement, de l'Aménagement et du Logement, DREAL) for both continental and overseas territories. All but 3 answered the questionnaire (23 positive responses) (Fig. 21).

Most regions have devoted a specific commission (CRPG) to this activity, mainly composed of geologists. Almost 300 geologists are involved in these commissions at the moment ( 26 regions have started their inventory to date). These members of the commissions belong to more than 40 institutions.

The inventory has been completed in 8 regions (all metropolitan) and 3 departments. To date, some 4,700 geosites are included; 3,400 are documented in full or partially, and more than 2,000 geosites are complete. At the moment (April 2015 ), 21\% of the informed geosites have stratigraphy as the first interest, $16 \%$ sedimentology, 16\% palaeontology, 12\% geomorphology, 9\%volcanism, 6\% natural resources, 6\% metamorphism, and so on. The percentage of main interest varies according to the geology of the region. For instance, volcanism is dominant in the Massif Central, whereas palaeontology is dominant in sedimentary basins. 


\section{Progress}

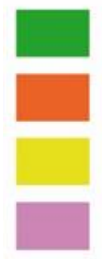

Validated national

In review

In progress

Not launched in 2014

Overseas departments and territories of

France :
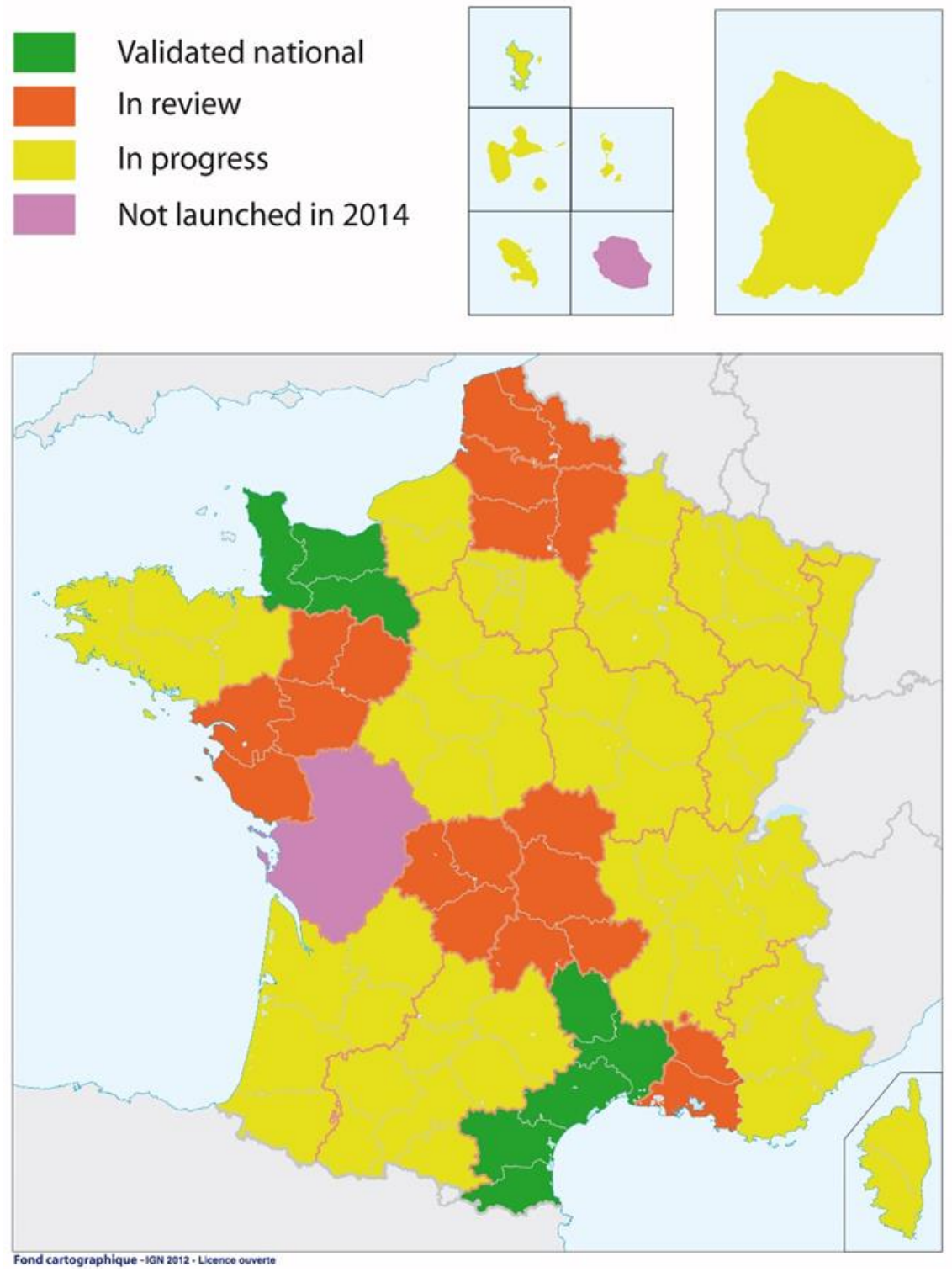

Fig. 21. Progress on the national inventory of geosites by region (as of February 2015). Three regions are already completed.

Regions that documented a restricted number of sites selected the most important ones and so they have a high proportion of 3 stars. In the Nord-Pas-de-Calais region, for instance, with about 60 geosites, there are no sites with 0 star, $18 \%$ with 1 star, $43 \%$ with 2 stars and $39 \%$ with 3 stars. In contrast, a region such as Midi-Pyrénées, with more than 1200 geosites, has $62 \%$ of its sites with zero star, $24 \%$ with 1 star, $10 \%$ with 2 stars, and $4 \%$ with 3 stars. Among the 2,000 geosites completed, $1 \%$ has no stars, $22 \%$ have 1 star, $43 \%$ have 2 stars and $34 \%$ have the 3 stars. As this national inventory is a continuous one, these regions will establish sites of lesser importance at a later date. Quite a variety of main interests exist between regions according to the geological context. Table 8 and Figure 22 show the main interest for the 2,000 geosites completed.

Table 8. Main interest reported for the French geosites as of spring 2015 
$\begin{array}{ll}\text { Stratigraphy } & 21 \%\end{array}$

Sedimentology $16 \%$

Palaeontology $16 \%$

Geomorphology $12 \%$

Volcanism $9 \%$

Natural resources $\quad 6 \%$

Metamorphism 6\%

Geochronology, hydrogeology, hydrothermalism, mineralogy, plutonism $14 \%$ and tectonics share the remaining.

\section{Main interests of actually completed sites (all regions, september 2014)}

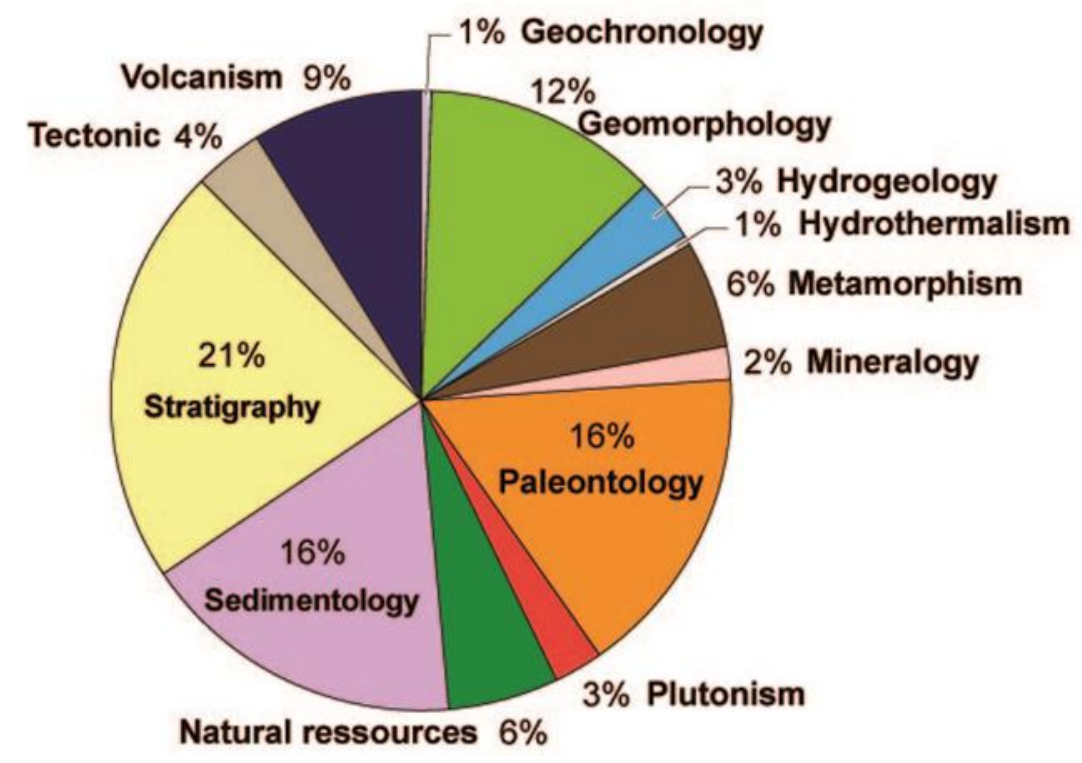

Fig. 22. Distribution of the main interest reported for the French geosites as of spring 2015

Table 8 and Figure 22 show that stratigraphy, sedimentology, and palaeontology are the main interest for most sites since they cover more than half of the sites (53\%).

\section{Who is involved?}

Besides the geologists and members of the commissions (CRPG and others), many other geoscientists are involved in the inventory process. The inventory has mobilized approximately 350 geologists belonging to 62 institutions (universities, regional administrations, companies, museums, education and so on), from all around France. 


\section{The need for an inventory; the SCAP}

In 2008, the French government set up a programme focused on the environment, called 'Grenelle de l'environnement'. This new focus encompasses a set of public or scientific actions dealing with the environment to be carried out in the entire country. One of the results indicated an insufficient presence of 'highly protected' areas. They currently represent $1 \%$ of the inland territory, and another $10 \%$ as slightly protected. In order to improve this situation, a strategy has been developed. This Stratégie de Création d'Aires protégées" (SCAP, strategy to create protected areas) was officially launched by the ministry in charge of the environment in 2009 to get a better idea of the protected areas network and establish a better representation of biodiversity and geodiversity. The ministry aims to place $2 \%$ of the French inland territory under stronger protection in the next ten years.

For geology, a specific group was organised by the government to analyse and propose geosites within this strategy, due to national inventory not yet being finished. For this purpose, several categories were distinguished.

- International standards (such as stratotypes or GSSP),

- Restricted sites (such as places with dinosaurs tracks or specific mineralogical content), - Main geological complexes (such as the ophiolitic complex of Mount Chenaillet in Alps),

- Landscapes important for their geomorphology (such as karstic countryside or the Gavarnie cirque in the Pyrenees).

An initial list with more than 140 major sites has been proposed on this basis and may benefit from stronger regulation. The implementation of this strategy is still ongoing. 


\section{V- Overview of some inventories in Europe}

Some European countries have a long history concerning the protection of geological heritage with well-developed strategies. Others have very limited legislation in this regard. One of the oldest known geological conservation case is in Germany, in the Baumannshöle cave where, from 1668, access for visitors was limited in order to preserve the site's stalactites and stalagmites (Erikstad, 2008).

In the early twentieth century, laws on nature protection sprang up in many European countries with more or less effect on the protection of geology. But it was not until the second half of the twentieth century that modern legislation, inventories and conservation strategies mainly developed. The creation of the European Association for the Conservation of Geological Heritage (ProGEO) in 1988 marked the beginning of the gathering and dissemination of information relating to geoconservation. In 2004, the protection of geological heritage was even included as a recommendation by the Council of Europe and is beginning to be visible in the policy of the European Union (Council of Europe, 2004).

To position the French inventory relative to what is done in other European countries, a comparative study was conducted in 2014. Four culturally different European countries were selected besides France: Spain, Finland, Great Britain and the Czech Republic (Fig. 23). The study focused on the motivations, context, actors, methodology, scale of the inventory, content of geological inventories, etc.. Particular attention was paid to criteria for selecting geological sites and to the various means of dissemination employed. 


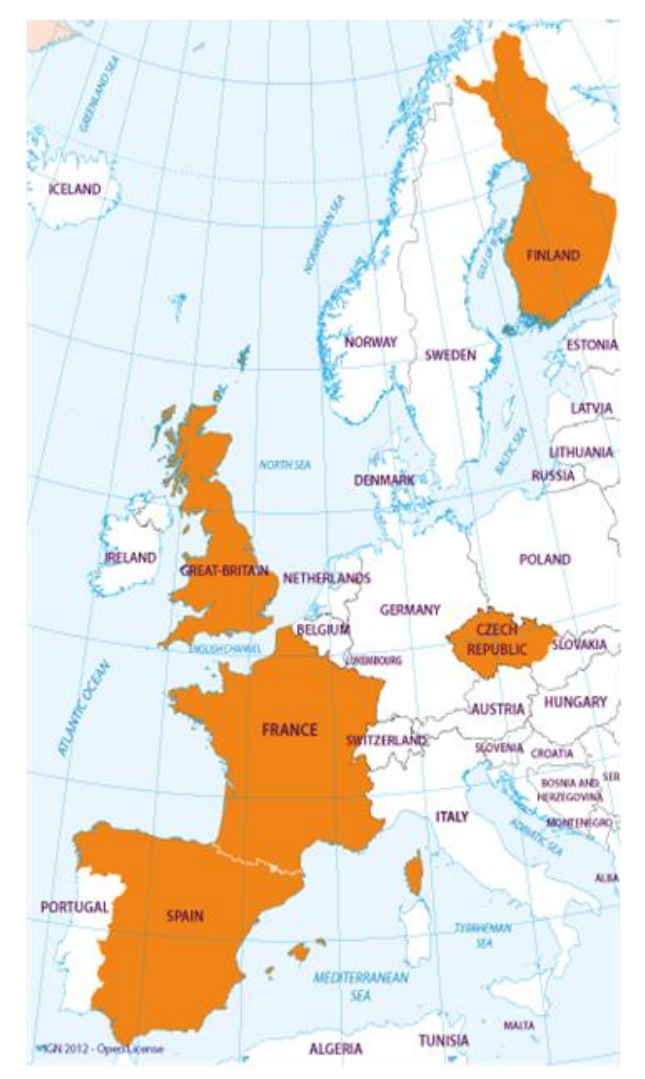

Fig. 23. Countries included in the study of inventories (IGN, 2012).

\section{Comparative study}

All five studied countries possess geological inventories with different names as shown in Table 9 below. Note that Great Britain has two separate inventories: the Geological Conservation Review (GCR) and Regionally Important Geological and Geomorphological Sites (RIGS). Also, the Finnish inventory was conducted in several stages, successively addressing different topics, which explains the various titles (Husa \& Teeriaho, 2004). The number of sites recorded in inventories varies depending on the country: from 1,500 sites expected in Spain to 4,717 sites listed in Finland. There are currently just over 611 confirmed sites in France and 305 in Spain, as these inventories are still underway.

Table 9. Names and number of sites in the studied inventories. Data for Spain from GarcíaCortés \& Carcavilla (2009), for Finland from the Finnish Environment Institute (2014), for 
France from De Wever et al.(2006), for Czech Republic from Wimbledon \& Smith-Meyer (2012) and for Great Britain from Ellis (2011) and GeoConservation UK (2014). In Northern Ireland, however, nationally important sites are recorded in the Earth Science Conservation Review (ESCR) and locally important sites are recognised as Sites of Local Nature Conservation Importance (SLNCI) (Wimbledon \& Smith-Meyer, 2012). The ESCR and SLNCI are the equivalent to the GCR and RIGS, respectively. For simplification matters, in this study, we will only use data from British inventories (GCR and RIGS).

\begin{tabular}{|c|c|c|}
\hline Country & Inventory & $\begin{array}{l}\text { Number of } \\
\text { sites }\end{array}$ \\
\hline Spain & Spanish Inventory of Sites of Geological Interest (IELIG) & $\begin{array}{c}1,500 \\
\text { (expected) }\end{array}$ \\
\hline \multirow[t]{4}{*}{ Finland } & National bedrock inventory & \multirow[t]{4}{*}{4717} \\
\hline & National inventory of moraine structures & \\
\hline & National inventory of coastal and wind formations & \\
\hline & National boulder field inventory & \\
\hline France & National inventory of geological heritage & $\begin{array}{c}4,700 \\
\text { (estimate) }\end{array}$ \\
\hline Czech Republic & Significant Geological Localities of The Czech Republic & 2799 \\
\hline \multirow[t]{2}{*}{ Great Britain $^{4}$} & Geological Conservation Review (GCR) & 3,000 \\
\hline & $\begin{array}{l}\text { Regionally Important Geomorphological and Geological } \\
\text { Sites (RIGS) }\end{array}$ & $\begin{array}{l}\text { Over 3,400 } \\
\text { (estimate) }\end{array}$ \\
\hline
\end{tabular}

Fig. 24 compares the number of geological sites depending on the size of each country, showing that the country where geological sites have the highest density is the Czech Republic. 


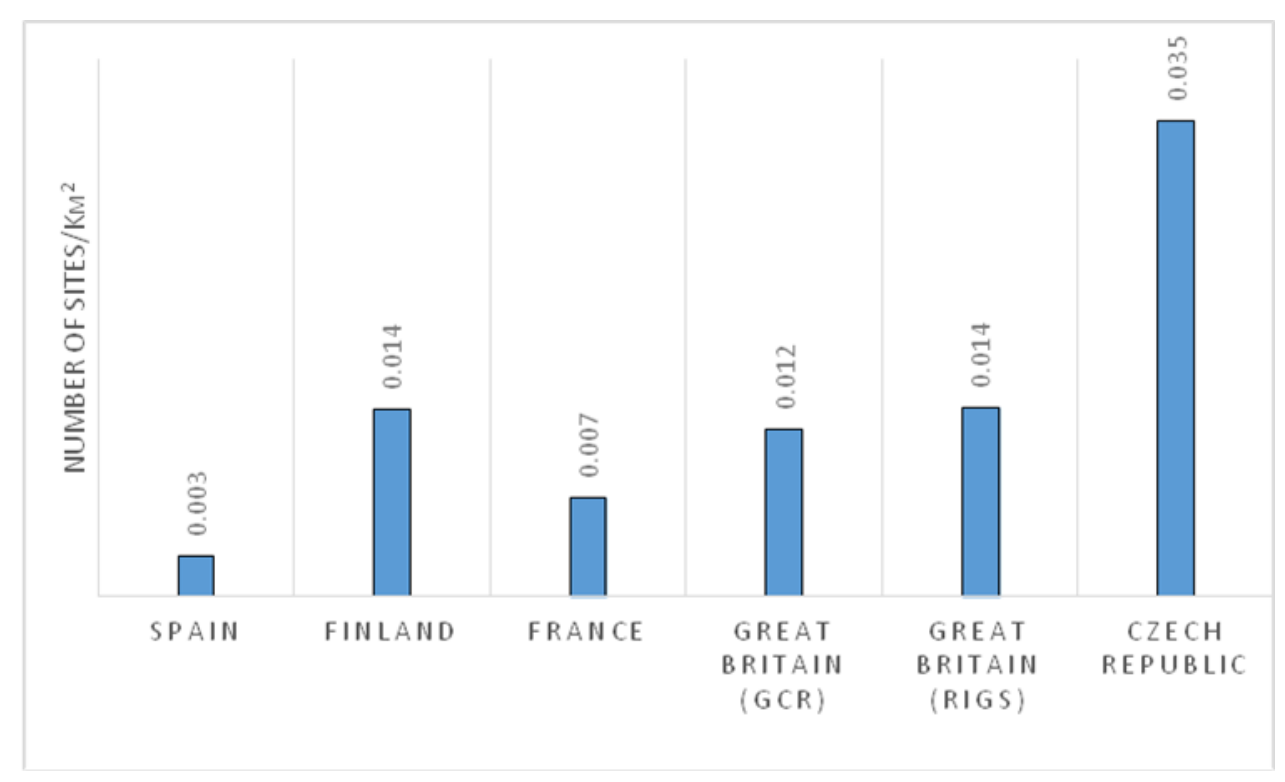

Fig. 24. Diagram showing the number of geological sites per $\mathrm{km}^{2}$. Inventories in progress are indicated by a dashed line.

\section{Origins and legal context}

Among modern inventories, the Geological Conservation Review, started in 1979 in Great Britain, is the first initiative of a systematic and comprehensive assessment of the geological heritage of a country (Ellis, 2011 - although earlier national site listings go back to the 1940s and the process of selecting RIGS sites effectively started in the 1970s). Finland, Spain and the Czech Republic launched their inventories in the late $20^{\text {th }}$ century, followed by France in the beginning of the $21^{\text {st }}$ century. In 2008, Spain published the results of its inventory of geosites of international significance (Global Geosites), and in 2009 revised its previous national methodology from 1978, later testing it at regional and local (municipal) scales (García-Cortés, 2012).

In most European countries, geological inventories result from nature and landscape protection laws. In Finland, however, the geological inventory was created in 1982 as the result of the Land Extraction Act MAL 551/1981. This law controls the excavation and exploitation of the ground and the use of bedrock as dimension stone and aggregate. The Land Extraction Act forbids the destruction of unique natural occurrences, whose uniqueness can be assessed on both geological and biological grounds. Thus, extraction is regulated in the sites assessed in the Finnish geological inventory. However, as long as extraction is not incompatible with the provisions of the act, an extraction permit can be granted (Finland's Ministry of Justice, 2014).

\section{Purpose and scale}

Setting goals is a crucial part of the methodological approach. The methodologies of geological inventories in Europe share three main objectives: 
- $\quad$ scientific knowledge,

- $\quad$ protection of geological heritage and,

- $\quad$ economic development of the local community.

A second observation shows that there are three types of inventories (Table 10): - organized around the preliminary identification of 'frameworks' characteristic of the geological history of a country. In Spain for geosites of international relevance, and in the GCR (Great Britain), sites of geological interest are not selected regardless of their context but in a geological 'framework' previously selected for its interest. These 'geological themes' or 'Selection Blocks' (e.g.: Marine Devonian stratigraphy' in the British GCR) are obtained by dividing up the geology and geomorphology of a country in several topics. These topics can be a regional geological phenomenon, stratigraphic series, a tectonic event, etc.. 'Frameworks' provide a structure for the selection of geological sites and ensure a balanced distribution of sites among the various Earth science aspects existing in the country (Ellis, 2011). Without using that name, it is also the rationale that was used to set up a list of sites for the National Strategy for the Creation of Protected Areas (SCAP) in France (Egoroff et al., 2011), e.g. carried out according to the processes which formed the geological formations (e.g. moraines, eolian deposits, etc, as in Finland; carried out regardless of representative geological frameworks or processes, as it is the case in France, Spain (local, regional and national inventories) and the Czech Republic, identifying sites of geological interest (sensu lato) for a general knowledge base.

We also note that inventories are carried out at different levels: international, national, regional or local (Table 10).

Table 10.Methodology types and scale of the studied inventories. Data for Spain from GarcíaCortés \& Carcavilla (2009), Agueda Villar et al., 2009, for Finland from Husa \& Teeriaho (2004), for France from De Wever et al. (2006), for Czech Republic from Kubalikova \& Kirchner (2013), for Great Britain from Ellis (2011) and Mason \& Stanley (2000).

\begin{tabular}{|c|c|c|}
\hline Country & Methodology type & Scale \\
\hline Spain & $\begin{array}{l}\text { Frameworks (for international) and } \\
\text { systematic inventory (for local, } \\
\text { regional and national) }\end{array}$ & $\begin{array}{l}\text { Local, regional, national and } \\
\text { international }\end{array}$ \\
\hline Finland & Nature of geological formation & National and regional \\
\hline France & Systematic inventory & National and regional \\
\hline Czech Republic & Systematic inventory & National and regional \\
\hline \multirow[t]{2}{*}{ Great Britain } & $\begin{array}{l}\text { GCR : Selection Blocks (i.e } \\
\text { 'frameworks') }\end{array}$ & National and international \\
\hline & $\begin{array}{lll}\text { RIGS : } & \text { Systematic } & \text { regional } \\
\text { inventory } & & \end{array}$ & Local, regional \\
\hline
\end{tabular}

\section{Selection criteria}

Once geological sites have been identified, selection criteria are applied to assess their interest as geoheritage. In general, three topics are addressed: scientific and educational interest, secondary interests (cultural, aesthetic, tourism...), and vulnerability of the site. Some criteria are common to all methodologies, such as criteria related to the main geological interest and the protection of the site. However, other criteria, such as the average temperature 
(weather criterion) in Spain or the number of colours in the landscape in the Czech Republic are scarcer (Kubalikova \& Kirchner, 2013). As part of a compatibility with a biotic natural heritage inventory, Spain, Finland, France and the Czech Republic, the inventories record the existence of biological interest or protected species within geological sites. In addition, it was found that the Spanish and French methodologies are those that have the most comprehensive selection process, with more than 40 fields to be filled in on their evaluation sheets, whereas other countries have between 20 and 30 .

Finally, in the five methodologies (Spain, France, Finland, Czech Republic and RIGS in Britain), the selected sites are scored and classified by degree of scientific importance and / or vulnerability. These assessments are intended to highlight the sites with the highest heritage interest, or requiring greater protection. However, the assessment of the vulnerability of a site, does not mean its legal protection. Only three of these methodologies (Finland, the Czech Republic and the GCR in Great Britain) include the listing of sites for their legal protection (Table 11) (although RIGS sites do have some status in development planning systems).

Table11. Types of protection applied to sites in the different inventories. Note. Data for Spain from GarcíaCortés \& Carcavilla (2009), Finland from Finland's Ministry of Justice (2014), France from De Wever et al. (2006), Czech Republic from Wimbledon \& Smith-Meyer (2012), and for the UK from Ellis (2011 - for the GCR) and from Mason \& Stanley (2000 - for RIGS).

\begin{tabular}{|l|l|}
\hline Inventory & \multicolumn{1}{|c|}{ Type of protection } \\
\hline Spain & $\begin{array}{l}\text { No direct protection. The inventory is used } \\
\text { by regional governments to define } \\
\text { protected areas. }\end{array}$ \\
\hline Finland & $\begin{array}{l}\text { Legal protection. Destruction and } \\
\text { damaging are forbidden. Excavation and } \\
\text { construction are restricted. }\end{array}$ \\
\hline France & $\begin{array}{l}\text { No direct protection. The inventory will be } \\
\text { used as the basis for the definition of future } \\
\text { protected areas (SCAP...) }\end{array}$ \\
\hline $\begin{array}{l}\text { Czech } \\
\text { Republic }\end{array}$ & $\begin{array}{l}\text { Legal protection. Fines for damage or } \\
\text { destruction (40-80,000€). }\end{array}$ \\
\hline $\begin{array}{l}\text { GCR (Great } \\
\text { Britain) }\end{array}$ & $\begin{array}{l}\text { Legal protection against destruction, } \\
\text { damage and neglect. }\end{array}$ \\
\hline $\begin{array}{l}\text { RIGS } \\
\text { (Great } \\
\text { Britain) }\end{array}$ & $\begin{array}{l}\text { Protection typical indirect, e.g. through } \\
\text { local planning processes. }\end{array}$ \\
\hline
\end{tabular}

\section{Means of dissemination}

Information gathered in geological inventories is frequently used to disseminate knowledge about geoheritage to the general public, scientists, nature conservation institutions and others. Multiple media are used: publication of descriptive sheets in books or on the internet or creation of online databases and interactive maps.

The first observation is that access to information is very uneven across countries. Much information is freely available on the internet. However, some descriptive sheets are published in expensive books with only incomplete information being available online 
(mainly legal documents only, e.g. the GCR in Britain) or even sold out (first reports in Finland); sometimes the databases only partially accessible to the general public (e.g. RIGS in Britain). In addition, the size of the disseminated sheets varies from one line (Spain) to approximately 8 pages (France) (Fig. 25). Some are very descriptive (GCR in Britain, Finland), others are restricted to keywords (Czech Republic, Spain). There is also a significant difference between the information provided on the assessment sheets of geosites and the information disseminated to the general public. The most obvious case is that of Spain, which has the largest number of selection criteria but only one line of description available for each site

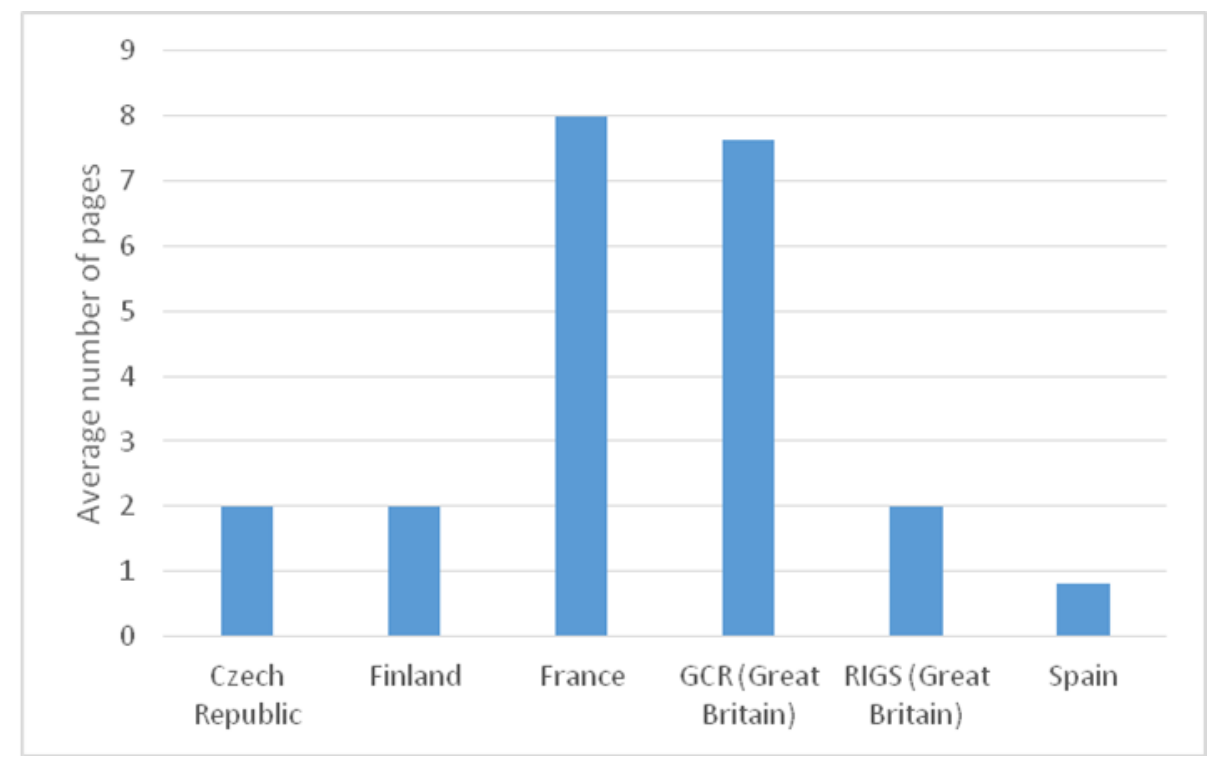

Fig. 25. Average number of pages for the disseminated descriptive sheets by country. Data for Czech Republic from the Czech Geological Survey (2014), Finland from the Finnish Environment Institute (2014), France from De Wever et al. (2006), for the GCR (UK) from the Joint Nature Conservation Committee (2008) and RIGS from GeoConservation UK (2014) and for Spain from García-Cortés et al. (2013).

Concerning the content, regardless of the inventory, the information included on each descriptive sheet is related to the location of the site and to its main geological interest. 'Secondary' interests do not appear on the disseminated descriptive sheets for Spanish and Czech inventories. Similarly, only Finland, France and the Czech Republic, and to a lesser extent RIGS in Great Britain, evoke vulnerability and protection on their final descriptive sheets (although for all nationally protected GCR sites, full management plans and conservation objectives have been compiled, but may not be publically readily accessible).

Illustrations are often attached to descriptive sheets, the most common being topographic maps and site plans, geological cross-sections, photographs and drawings. The boundaries of protected areas that include nationally protected GCR sites and most RIGS sites are, however, availability as GIS layers.

The results of the comparative study of geological inventory methodologies are summarized in the following Table 12 
Table 12. Synthetic table. Data for Spain from García-Cortés \& Carcavilla (2009), for Finland from the Finnish Environment Institute (2014), for France from De Wever et al. (2006), for the Czech Republic from Wimbledon \& Smith-Meyer (2012) and Kubalikova \& Kirchner (2013), for GCR from Ellis (2011) and for RIGS from Mason \& Stanley (2000). 


\begin{tabular}{|c|c|c|c|c|c|c|}
\hline & SPAIN & FINLAND & FRANCE & CZECH REPUBLIC & $\begin{array}{l}\text { GREAT BRITAIN } \\
\text { (excluding } \\
\text { Northern Ireland) }\end{array}$ & GREAT BRITAIN \\
\hline & & & & & GCR (SSSI) & RIGS \\
\hline \multirow[t]{4}{*}{ NAME } & $\begin{array}{l}\text { Spanish national } \\
\text { inventory of sites of } \\
\text { geological interest } \\
\text { (1978-1988 and } \\
2007 \text { onwards). }\end{array}$ & $\begin{array}{c}\text { National bedrock } \\
\text { inventory, 1989- } \\
2004 .\end{array}$ & $\begin{array}{c}\text { National inventory } \\
\text { of geological } \\
\text { heritage, } 2007 .\end{array}$ & $\begin{array}{c}\text { Significant } \\
\text { Geological } \\
\text { Localities of The } \\
\text { Czech Republic, } \\
1992\end{array}$ & $\begin{array}{c}\text { Geological } \\
\text { Conservation } \\
\text { Review (GCR), } \\
1979 .\end{array}$ & $\begin{array}{c}\text { Regionally } \\
\text { Important } \\
\text { Geological and } \\
\text { Geomorphological } \\
\text { Sites (RIGS), } 1990 \\
\text { (although some } \\
\text { regional selections } \\
\text { go back to the } \\
\text { 1970s). }\end{array}$ \\
\hline & $\begin{array}{c}\text { Several local and } \\
\text { regional } \\
\text { inventories. }\end{array}$ & $\begin{array}{c}\text { National inventory } \\
\text { of moraine } \\
\text { structures, } 2007 .\end{array}$ & & & & \\
\hline & $\begin{array}{l}\text { Spanish geosites of } \\
\text { international } \\
\text { relevance (1998- } \\
\text { 2008) }\end{array}$ & $\begin{array}{l}\text { Nation inventory of } \\
\text { coastal and wind } \\
\text { formations, 2005- } \\
2009 \text {. }\end{array}$ & & & & \\
\hline & & $\begin{array}{c}\text { National boulder } \\
\text { field inventory, } \\
2012 \text {. }\end{array}$ & & & & \\
\hline CONTEXT & $\begin{array}{c}\text { Law } 42 / 2007 \text { about } \\
\text { natural heritage and } \\
\text { biodiversity }\end{array}$ & $\begin{array}{l}\text { MAL 551/1981 } \\
\text { Land Extraction Act } \\
\text { (articles } 3 \text { and 7) }\end{array}$ & $\begin{array}{l}\text { Law } 27 / 022002 \text { on } \\
\text { local democracy }\end{array}$ & $\begin{array}{l}\text { Law } 114 / 1992 \text { Coll. } \\
\text { on nature and } \\
\text { landscape } \\
\text { protection }\end{array}$ & $\begin{array}{c}\text { Wildlife and } \\
\text { Countryside Act } \\
1981 \text {; Countryside } \\
\text { and Rights of Way } \\
\text { Act } 2000 \text { in } \\
\text { England and Wales } \\
\text { (see Page and } \\
\text { Wimbledon } 2009 \text { for } \\
\text { regional differences) }\end{array}$ & $\begin{array}{l}\text { Various elements of } \\
\text { national and local } \\
\text { spatial planning } \\
\text { legislation. }\end{array}$ \\
\hline
\end{tabular}




\begin{tabular}{|c|c|c|c|c|c|c|}
\hline & SPAIN & FINLAND & FRANCE & CZECH REPUBLIC & $\begin{array}{l}\text { GREAT BRITAIN } \\
\text { (excluding } \\
\text { Northern Ireland) }\end{array}$ & GREAT BRITAIN \\
\hline & & & & & & RIGS \\
\hline GOAL & $\begin{array}{c}\text { Selection, } \\
\text { description and } \\
\text { assessment of sites } \\
\text { of geological } \\
\text { interest towards } \\
\text { their management } \\
\text { and protection. }\end{array}$ & $\begin{array}{l}\text { Identify remarkable } \\
\text { sites for biological, } \\
\text { geological and } \\
\text { landscape interest } \\
\text { from a national } \\
\text { point of view or for } \\
\text { the protection of the } \\
\text { environment }\end{array}$ & $\begin{array}{l}\text { Identify all the sites } \\
\text { and objects of } \\
\text { geological interest, } \\
\text { in situ and ex situ. } \\
\text { Collect and enter } \\
\text { their characteristics } \\
\text { on appropriate } \\
\text { forms. Rank and } \\
\text { validate heritage } \\
\text { oriented sites. } \\
\text { Assess their } \\
\text { vulnerability and } \\
\text { protection needs. }\end{array}$ & $\begin{array}{l}\text { Compile field data } \\
\text { on major geological } \\
\text { sites to inform the } \\
\text { general public and } \\
\text { provide information } \\
\text { to nature protection } \\
\text { institutions. }\end{array}$ & $\begin{array}{l}\text { Identify sites of } \\
\text { national and } \\
\text { international } \\
\text { importance which } \\
\text { constitute scientific } \\
\text { key elements of } \\
\text { British geoheritage } \\
\text { (excluding Northern } \\
\text { Ireland). }\end{array}$ & $\begin{array}{l}\text { Locally identify sites } \\
\text { of local, regional or } \\
\text { national interest, for } \\
\text { geodiversity (e.g. at } \\
\text { a county level). }\end{array}$ \\
\hline SCALE & $\begin{array}{l}\text { Local, regional, } \\
\text { national and } \\
\text { international }\end{array}$ & $\begin{array}{l}\text { National and } \\
\text { regional }\end{array}$ & $\begin{array}{l}\text { National and } \\
\text { regional }\end{array}$ & $\begin{array}{l}\text { National and } \\
\text { regional }\end{array}$ & $\begin{array}{l}\text { National and } \\
\text { international }\end{array}$ & $\begin{array}{l}\text { Local, regional and } \\
\text { national }\end{array}$ \\
\hline $\begin{array}{c}\text { MAIN } \\
\text { STAKEHOLDERS }\end{array}$ & $\begin{array}{l}\text { Geological Survey } \\
\text { of Spain (IGME), } \\
\text { regional and local } \\
\text { administrations }\end{array}$ & $\begin{array}{c}\text { Finnish } \\
\text { Environment } \\
\text { Institute (SYKE) } \\
\text { and Geological } \\
\text { Survey of Finland } \\
\text { (GTK) }\end{array}$ & $\begin{array}{c}\text { French Ministry of } \\
\text { Environment- } \\
\text { DREAL (regional } \\
\text { State } \\
\text { representative)- } \\
\text { French National } \\
\text { Museum of Natural } \\
\text { History }\end{array}$ & $\begin{array}{l}\text { Czech Geological } \\
\text { Survey (CGS) }\end{array}$ & $\begin{array}{c}\text { 'Country' } \\
\text { conservation } \\
\text { agencies, Joint } \\
\text { Nature } \\
\text { Conservation } \\
\text { Committee (JNCC) }\end{array}$ & $\begin{array}{c}\text { The Royal Society of } \\
\text { Wildlife Trusts } \\
\text { (RSWT)/ local RIGS } \\
\text { groups and Geology } \\
\text { Trusts, local area } \\
\text { government } \\
\text { organisations, (most } \\
\text { are affiliated to } \\
\text { GeoConservationUK) }\end{array}$ \\
\hline $\begin{array}{l}\text { METHODOLOGY } \\
\text { TYPE }\end{array}$ & $\begin{array}{l}\text { Systematic (local, } \\
\text { regional and } \\
\text { national) and } \\
\text { frameworks } \\
\text { (international) }\end{array}$ & $\begin{array}{l}\text { Nature of } \\
\text { geological } \\
\text { formation }\end{array}$ & Systematic & Systematic & $\begin{array}{l}\text { Framework/ } \\
\text { Systematic }\end{array}$ & Systematic \\
\hline
\end{tabular}




\begin{tabular}{|c|c|c|c|c|c|c|}
\hline & SPAIN & FINLAND & FRANCE & CZECH REPUBLIC & $\begin{array}{l}\text { GREAT BRITAIN } \\
\text { (GCR) (excluding } \\
\text { Northern Ireland) }\end{array}$ & $\begin{array}{l}\text { GREAT BRITAIN } \\
\text { (RIGS) }\end{array}$ \\
\hline $\begin{array}{l}\text { SELECTION } \\
\text { CRITERIA }\end{array}$ & $\begin{array}{c}\text { Scientific, } \\
\text { educational, } \\
\text { touristic and } \\
\text { protection interests }\end{array}$ & $\begin{array}{c}\text { Geological- } \\
\text { geomorphological } \\
\text { (representativeness, } \\
\text { rarity, diversity), } \\
\text { biological, } \\
\text { ecological, } \\
\text { landscape, and } \\
\text { environmental } \\
\text { interests }\end{array}$ & $\begin{array}{l}\text { Representativeness } \\
\text { educational and } \\
\text { scientific interests, } \\
\text { rarity, protection } \\
\text { status, additional } \\
\text { interests }\end{array}$ & $\begin{array}{l}\text { Scientific value, } \\
\text { relationship with the } \\
\text { biosphere, cultural } \\
\text { and historical links, } \\
\text { attractiveness }\end{array}$ & $\begin{array}{l}\text { International } \\
\text { importance, } \\
\text { presence of } \\
\text { exceptional objects, } \\
\text { representativeness }\end{array}$ & $\begin{array}{c}\text { Geodiversity, } \\
\text { educational and } \\
\text { geological, cultural, } \\
\text { economic, heritage } \\
\text { interests, access } \\
\text { notes and security }\end{array}$ \\
\hline $\begin{array}{c}\text { NUMBER OF } \\
\text { SITES }\end{array}$ & 1,500 (expected) & 4,717 & 4,700 (expected) & 2,799 & 3,000 & $\begin{array}{c}\text { More than 3,000 } \\
\text { (estimate) }\end{array}$ \\
\hline $\begin{array}{l}\text { DISSEMINATION } \\
\text { MEDIUM }\end{array}$ & $\begin{array}{l}\text { Database, reports } \\
\text { and publications }\end{array}$ & Reports & Database & Database & $\begin{array}{l}\text { Database and } \\
\text { books }\end{array}$ & $\begin{array}{l}\text { Database (although } \\
\text { in some areas site } \\
\text { details - may not be } \\
\text { readily available) } \\
\text { and publications }\end{array}$ \\
\hline PROTECTION & $\begin{array}{l}\text { No protection from } \\
\text { the inventory } \\
\text { (competence of } \\
\text { regional and local } \\
\text { administration) }\end{array}$ & $\begin{array}{l}\text { Legal protection. } \\
\text { Restricted } \\
\text { extraction and } \\
\text { construction }\end{array}$ & No protection & $\begin{array}{l}\text { Legal protection of } \\
\text { geosites. Fines in } \\
\text { case of damage or } \\
\text { destruction (40€- } \\
80,000 €) \text {. }\end{array}$ & $\begin{array}{l}\text { Legal protection } \\
\text { against } \\
\text { construction, } \\
\text { damage and } \\
\text { dereliction }\end{array}$ & $\begin{array}{l}\text { Protection mainly } \\
\text { through local } \\
\text { planning systems }\end{array}$ \\
\hline $\begin{array}{c}\text { INTEGRATION OF } \\
\text { BIOLOGY }\end{array}$ & No & Part of the inventory & Complementarity & In the evaluation & $\begin{array}{l}\text { Integration within } \\
\text { designated } \\
\text { protected sites }\end{array}$ & No \\
\hline $\begin{array}{c}\text { NUMERICAL } \\
\text { EVALUATION OF } \\
\text { SITES }\end{array}$ & Yes & Yes & Yes & Yes & No & $\begin{array}{l}\text { Some networks } \\
\text { only. }\end{array}$ \\
\hline $\begin{array}{l}\text { EVALUATION OF } \\
\text { PROTECTION } \\
\text { NEEDS }\end{array}$ & Yes & No & Yes & Yes & Yes & Yes \\
\hline
\end{tabular}




\section{Main similarities and differences}

At first, when looking at each inventory, we note that four of them have the word 'inventory' or a synonym (i.e. review) in their name (Finland, France, Sapine-IELIG- and GCR in Great Britain). Concerning geoheritage vocabulary, we find that different methodologies use similar words (inventory, geosite ...) but with different meanings. For example, in France, a geosite is the "bounded space that offers the opportunity to observe the geological elements and / or events which have an interest for the understanding of Earth Sciences" (De Wever et al. 2006). In contrast, in the GCR (Great Britain) and in Spain, geosites are restricted to "sites of national or international interest used to describe the key elements of the history of geology" (Ellis, 2011) of the involved country. Thus, this difference of meaning has a direct impact on the number of geological sites included each inventory.

To this difference of meaning must be added the link between the objectives and the types of methodologies. The 'frameworks' methodology is used to review nationally or internationally important geosites (Spain, GCR in Great Britain). In this type of organisation, 'frameworks', typical aspects of the geological history of the country, are defined first, and then geosites that best represent these frameworks are chosen (Agueda Villar et al., 2009), often limiting the number of geosites due to a perceived need to avoid duplication or less representative sites. In contrast, in inventories carried out more comprehensively in order to develop a 'knowledge base' and conducted at a national and / or regional scale (France, Finland, Czech Republic, RIGS in Britain, Spain), the number of sites is higher.

There is also a link between the selection and/or evaluation criteria and the purpose of the inventory. When the emphasis is on geological or educational interest and the vulnerability of a site, the aim of the inventory is to gather information about geological heritage and also to provide solutions for the protection of this heritage. This is the case for French, Finnish, Spain and the GCR (UK excluding Northern Ireland) inventories. However, some inventories (Spain, Czech Republic, most RIGS, UK), in addition to gathering knowledge about geoheritage, attach importance to conditions of access to the site, potential use of the site, already existing infrastructure, etc.. One of their aims is to contribute to the economic development of a region or a locality.

Britain constitutes a particular case because it has two separate inventories. The oldest, the Geological Conservation Review, as previously mentioned, is a list of geological sites of national or international importance representative of the history of British geology (Ellis, 2011). All the sites listed in the GCR in Britain are under legal protection. However, this inventory excludes many geological sites of lower importance. Scientists and volunteers, alerted by the deterioration of some sites not listed in the GCR, decided to establish the concept of 'RIGS' "to inventory local, regional or national sites of interest for geodiversity" (Mason \& Stanley, 2000). It is a small-scale initiative, coordinated by NGO associations (such as GeoConservationUK), while the GCR is managed by the Joint Nature Conservation Committee, on behalf of 'country' conservation agencies, funded by the British government. Both inventories are completely independent in terms of their organization, their goals and their methodology. However, this does not prevent geological sites from overlapping: a nationally important site may also have a more local interest.

\section{Development prospects}


Behind the term 'geological inventory' there are several different entities. Each country sets up a specific inventory in order to meet its objectives and its needs in terms of geoheritage conservation; there are as many inventories as countries, or even more in the case of Great Britain and Spain. This multitude of approaches and strategies makes comparisons difficult. One thing is sure, the process of carrying out geological inventories in Europe is well underway since, at the present time, the majority of European countries have launched or are planning to launch a geological inventory (Wimbledon \& Smith-Meyer, 2012) (Fig. 26). However, in the context of a possible geological inventory project on a European or global scale, given the methodological differences between the various national inventories, the issue of interoperability of the different inventories will arise. Harmonization work would be necessary in order to obtain a coherent and homogeneous data set at an international level (for instance in the context of the Global Geosites project).

Fig. 26. State of progress of national geological inventories in Europe with the number of geosites up to October 2014. Data from Wimbledon \& Smith-Meyer(2012) and see Appendix 3 for additional sources. Mapping from IGN, 2012.

[insert]

\section{VI- Outreach}

\section{Knowledge and geoconservation}

The inventory is a preliminary work, essential for understanding geological heritage (and therefore natural heritage), but it is not its sole interest. The methodology allows the evaluation of the registered sites, the identification of the heritage value and protection needs of each site. It constitutes a reference for spatial planning policies and for the definition of conservation strategies and the enhancement of this heritage, in various ways, both regionally and nationally or even internationally, by providing a tool / program able to rank sites of geological interest across the territory, at multiple levels of needs (heritage, protection etc.).

The geoheritage inventory is a tool of knowledge that has no legal value. However, it is used to notify the various administrations and local authorities, particularly municipalities: it mentions the elements to take into account when preparing their planning documents. It indicates the presence of outstanding geological sites that need special attention as required by the 2002 Act in France. This acknowledgment can prevent the destruction of the sites due to ignorance, as has sometimes been the case (Fig. 27). 


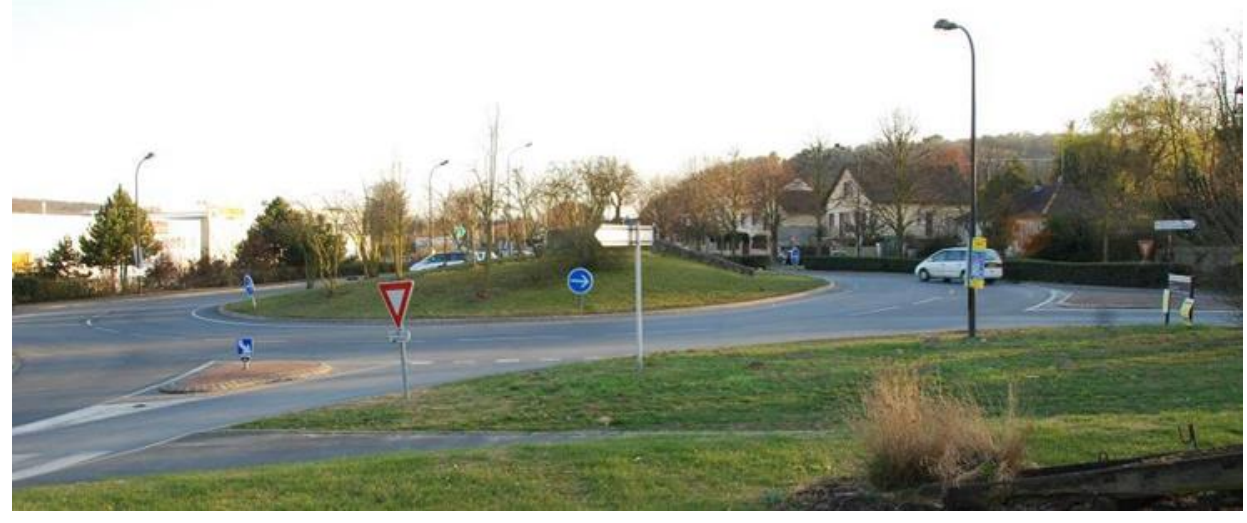

Fig. 27. A roundabout constructed on an outcrop of the 'Falun d'Etrechy', part of the historical Stampian stratotype, but nevertheless ignored by the local planning authorities (photo: P. De Wever)

This inventory provides a criterion to identify areas of higher density of geosites as important sites, and identify the priorities to determine protected areas as part of geoconservation plans as well as choosing the best protection tool (Fig. 28 and 29).

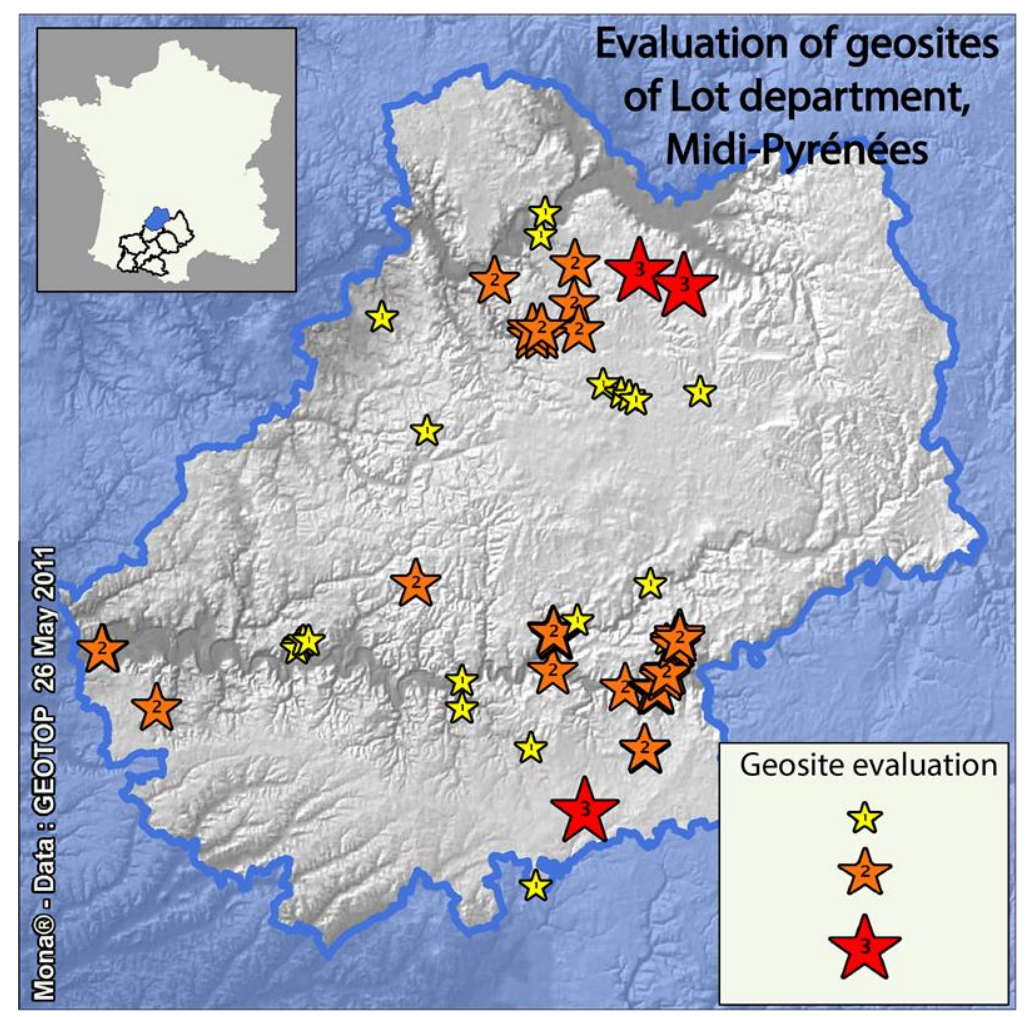

Fig. 28. Map of part of a region with: (a) local geosite evaluation and (b) the main geological interest of the geosites (tectonic / sedimentary / paleontological, etc.). 


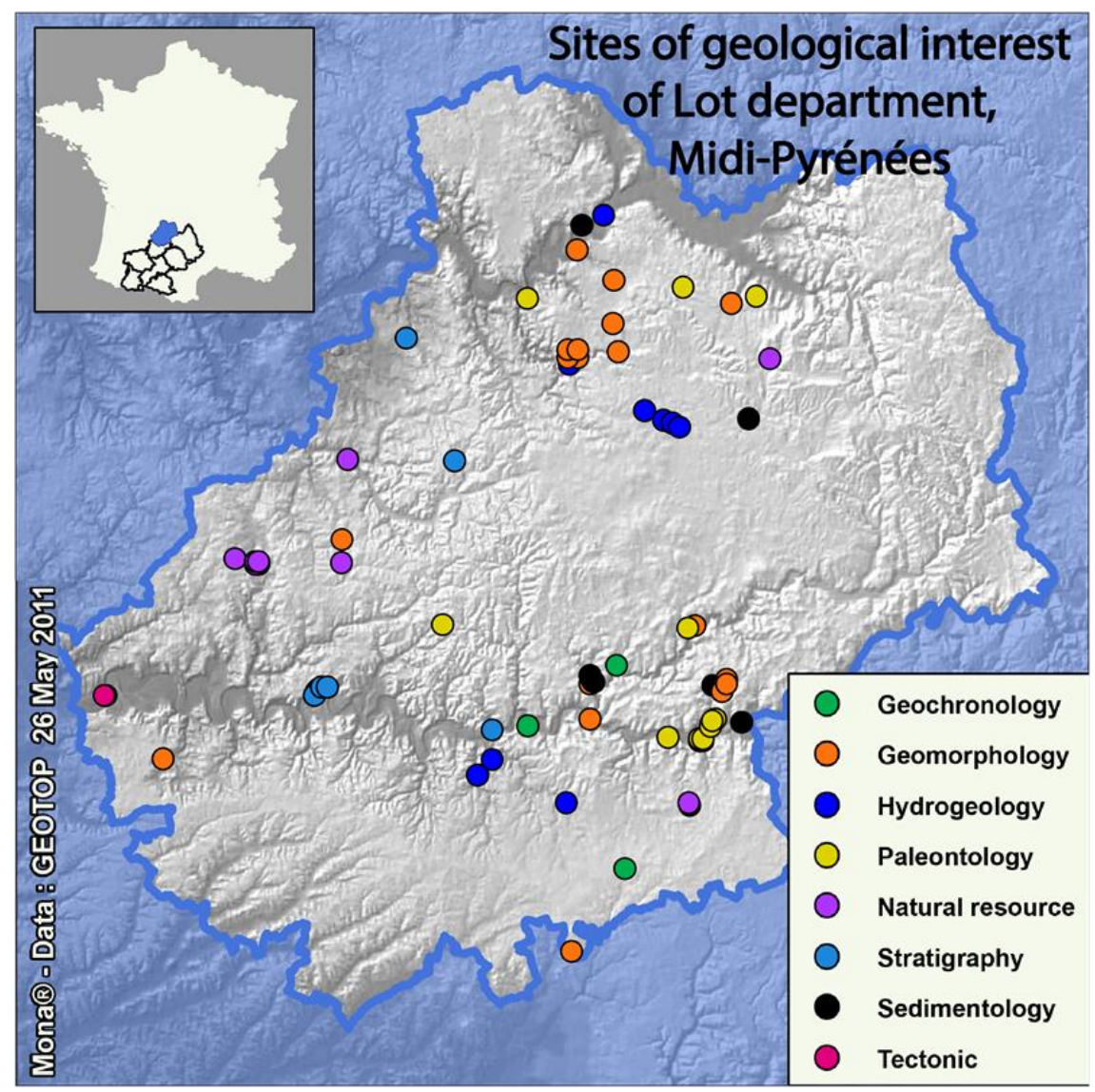

Fig. 29. Automatically generated regional report map displaying various densities of geosites.

\section{Integration into a global information system on nature and landscape and data dissemination}

Together with the other programs of knowledge of natural heritage, the INPG (National inventory of geological heritage) helps to promote knowledge of nature, whether they are inventories such as ZNIEFFs (natural areas of ecological interest, flora and fauna) or evaluation networks such as Natura 2000 sites or red species lists (for more details see the INPN (National inventory of natural heritage) at http://inpn.mnhn.fr). The INPN provides data on nature for policy makers, conservation stakeholders, researchers, etc, and the general public. These are the challenges of major national, European and international programs for the protection of nature. In France, data from these programmes feed the 'Information System on Nature and Landscapes' (SINP). This information system is a partnership between the ministry in charge of the environment, public institutions, associations and local authorities involved in the production, validation, management, processing, enhancement and dissemination of information on nature or landscape.

The official tool for disseminating data on nature collected in this context is the INPN website, managed by the French National Museum of Natural History (MNHN). The INPN (http://inpn.mnhn.fr) is the 'reference system' of the SINP.

The mapping of each site of the inventory allows the representation of the outstanding sites of geological interest, along with data from other nature inventories on the whole territory. One can display on a map of the territory, information concerning the geology, as 
well as the flora, or both, and compare them. This is a major advantage of this 'national' and multi-layer dimension of the inventory.

\section{Use and development of the inventory}

The promotion of geological sites can be done in different ways and is aimed at a variety of audiences:

- The data from the inventory provides resources to supply a database, called 'lithothèque nationale', dedicated to education, particularly for secondary school teachers, in order to provide them with an effective way to set up field trips for their students (Fig. $30 \& 31$ ).

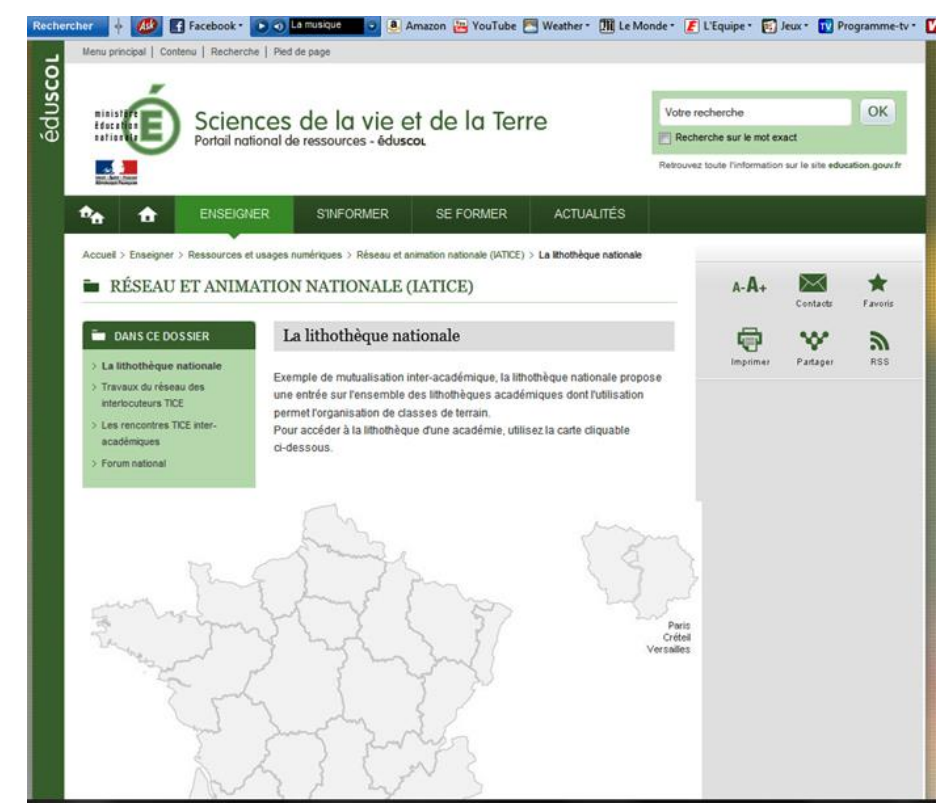

Fig. 30. Home page of the 'lithothèque' website for geosites, dedicated to education on the (from EducScol - http://eduscol.education.fr/svt/enseigner/ressources-et-usagesnumeriques/reseau-et-animation-nationale-iatice/la-lithotheque-nationale.html).

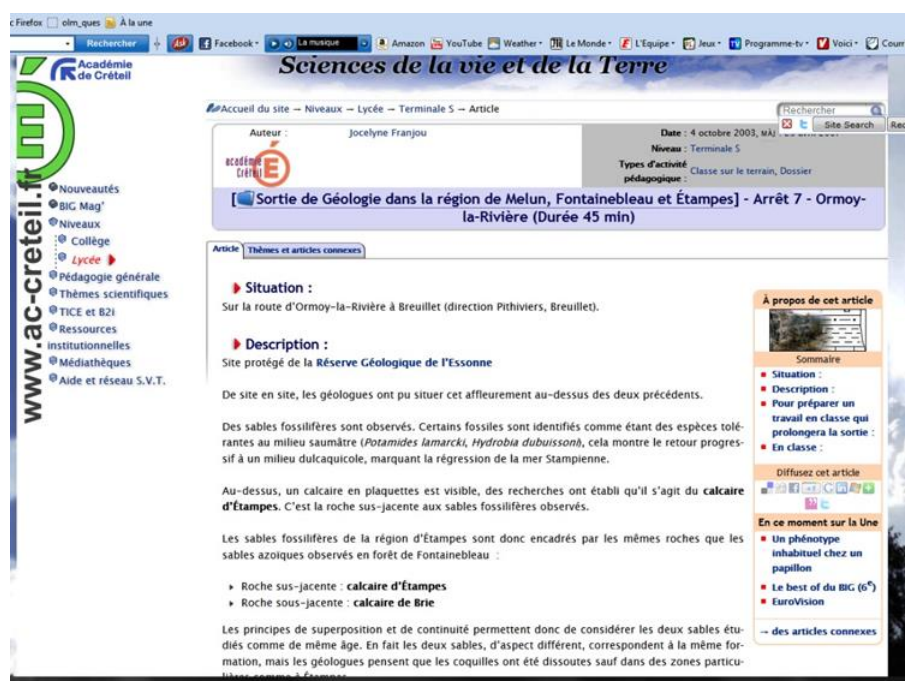


Fig. 31. Example of the lithothèque' webpage for the Stampian.

- Recent years have seen the rise of publications intended to raise awareness of geology and geological heritage, aimed at a wider audience, some directly related to the creation of the inventory (e.g., Jonin, 2008; Robaszinsky and Guyétant, 2009). Recently, several new collections were launched: "Stratotypes" (stratotypes) (Fig. 32), "Balades géologiques" (geological strolls) (Fig. 33), "Géotourisme" (geotourism) (Fig. 34), "Guides géologiques” (geological guides).

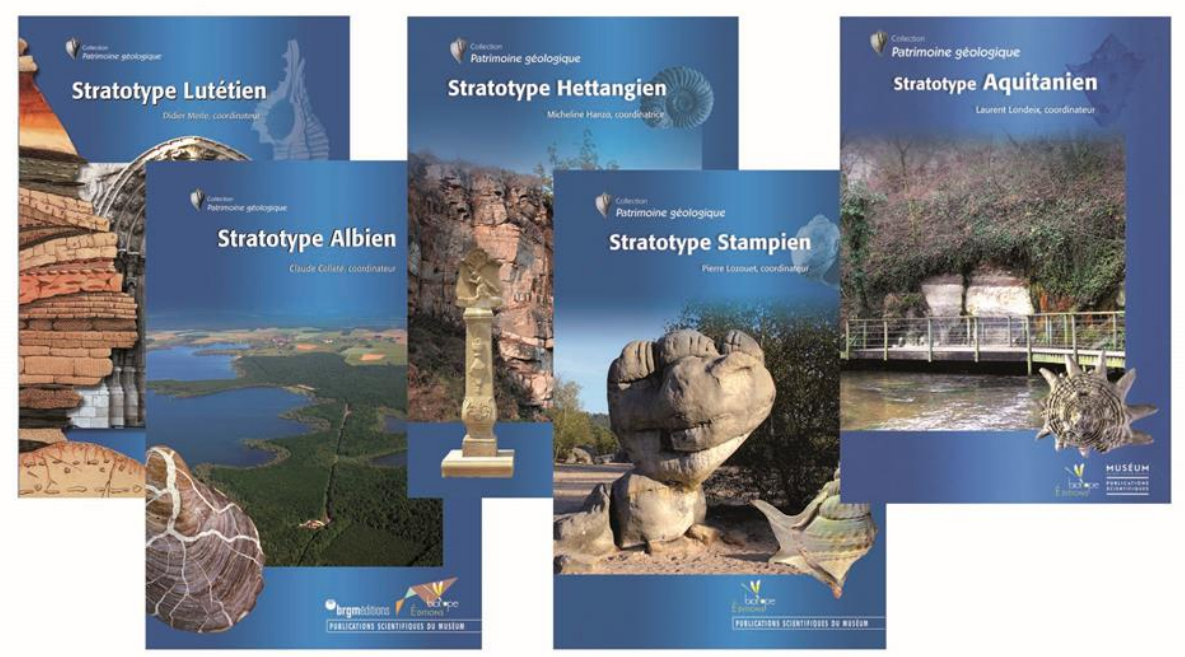

Fig. 32. Covers of the first 5 books dealing with stratotypes: Lutetian (Merle, 2008), Albian (Colleté, 2010), Hettangian (Hanzo, 2012), Stampian (Lozouet, 2012) and Aquitanian (Londeix, 2014). The last one has received the patronage of UNESCO. Launched by the French National Museum of Natural History, the collection "Patrimoine géologiqueStratotypes" (Geoheritage-Stratotypes) aims to explain what a stratotype is, namely a scientific standard of international value, and to increase awareness of the value of this heritage.
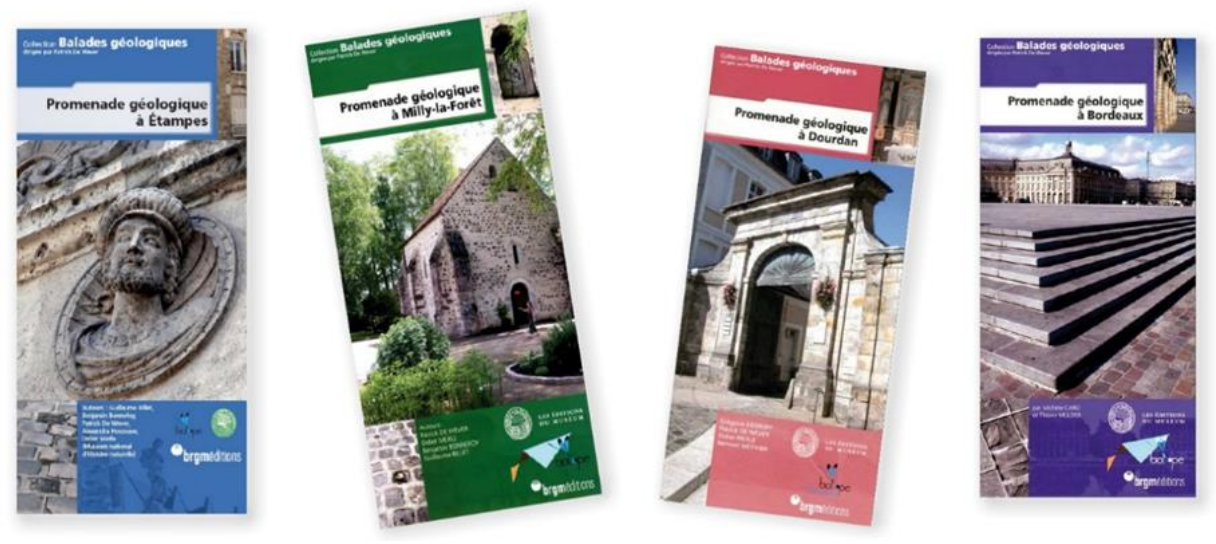

Fig. 33. Examples of geological itineraries: Etampes (Billet et al., 2008), Milly-la-Forêt (De Wever et al., 2009), Dourdan (Egoroff et al,. 2011) and Bordeaux (Caro et al., 2010). The booklets in the 'Balades Géologiques' (geological itineraries) collection, created by the French National Museum of Natural History, describe geological city tours that show the 
relationships between rocks, architecture, city planning and history, combining art and science.

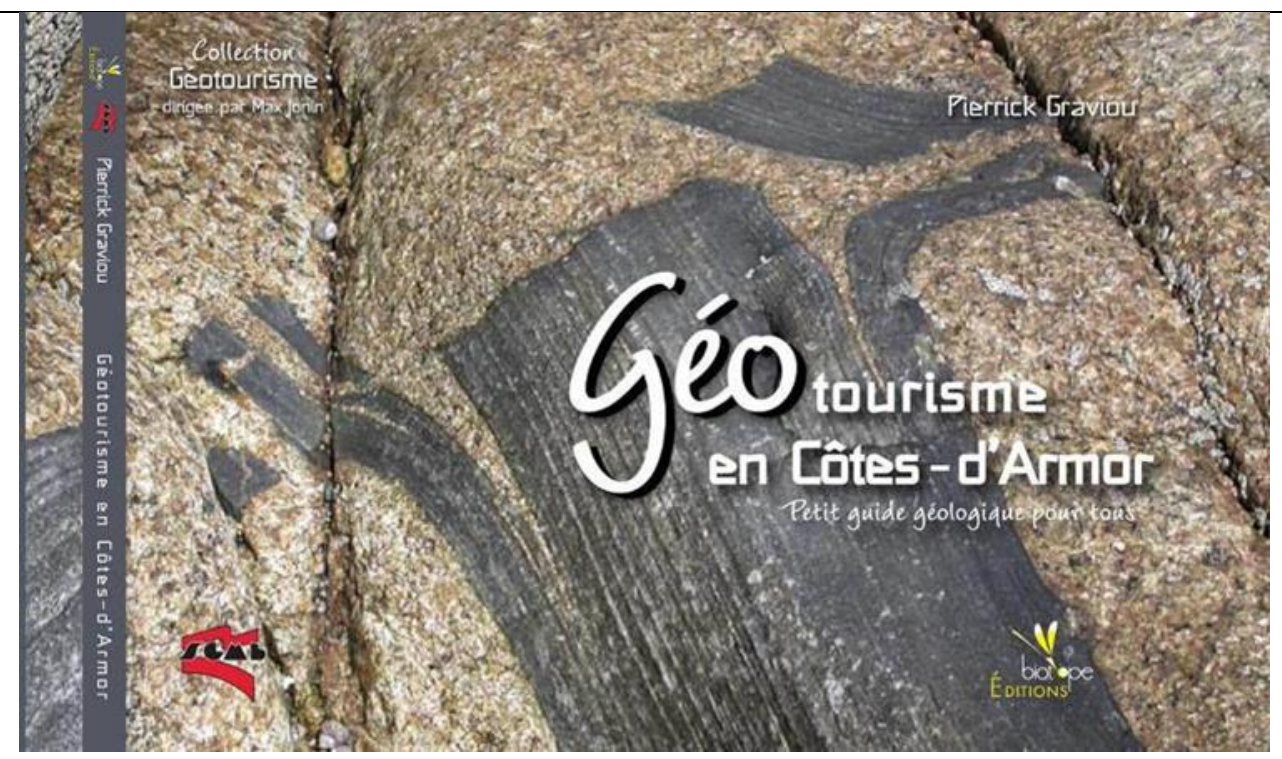

Fig. 34. 'Géotourisme' collection: small books that present geosites by department. Here is the book on "Côtes-d'Armor" (Graviou, 2012).

- An awareness and a better understanding of geology is also shown through the development of sites and geological tours or routes. Geological trails or roads provide access to in situ geology at different scales: on distances covered by foot or by car. Several road tours have been developed with this objective. e.g. Géoroute du Chablais (Chablais geological road), Via GeoAlpina and the Route géologique transpyrénéenne (trans-Pyrenean geological road) (Fig. 35 and 36).

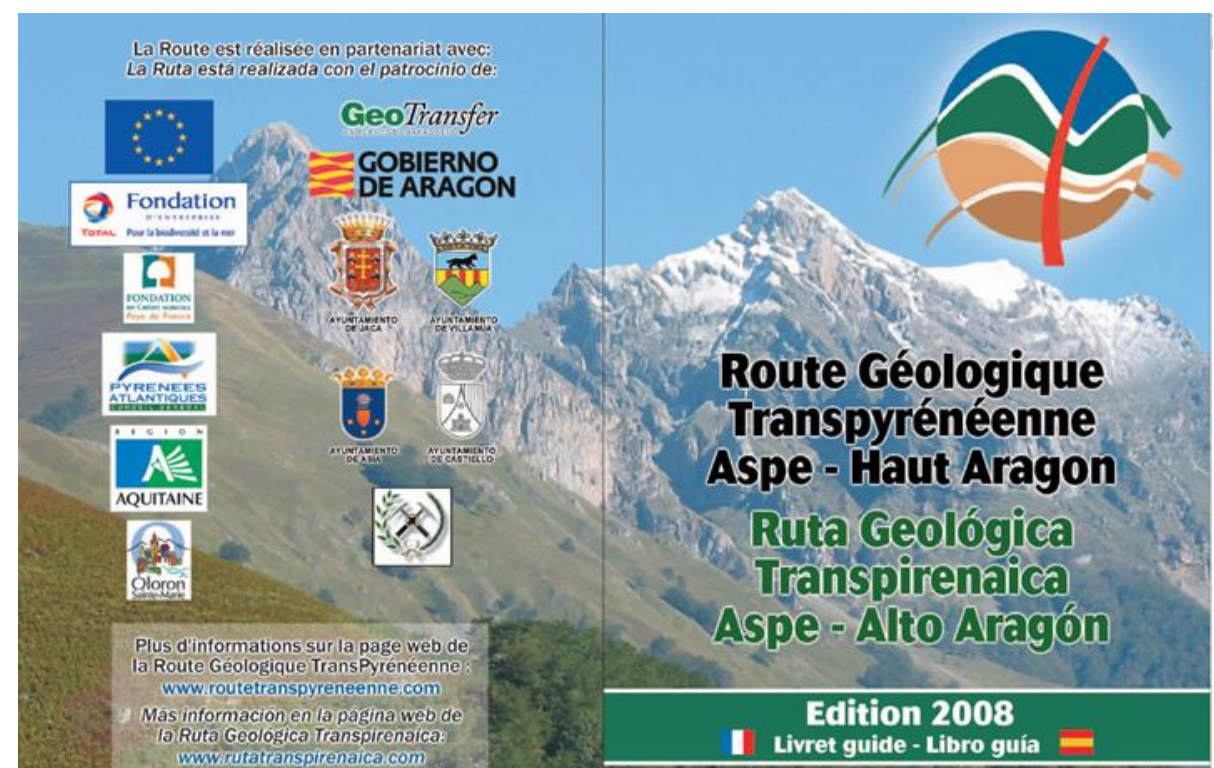

Fig. 35. Cover of the guidebook of the Route Géologique Transpyrénéenne. 


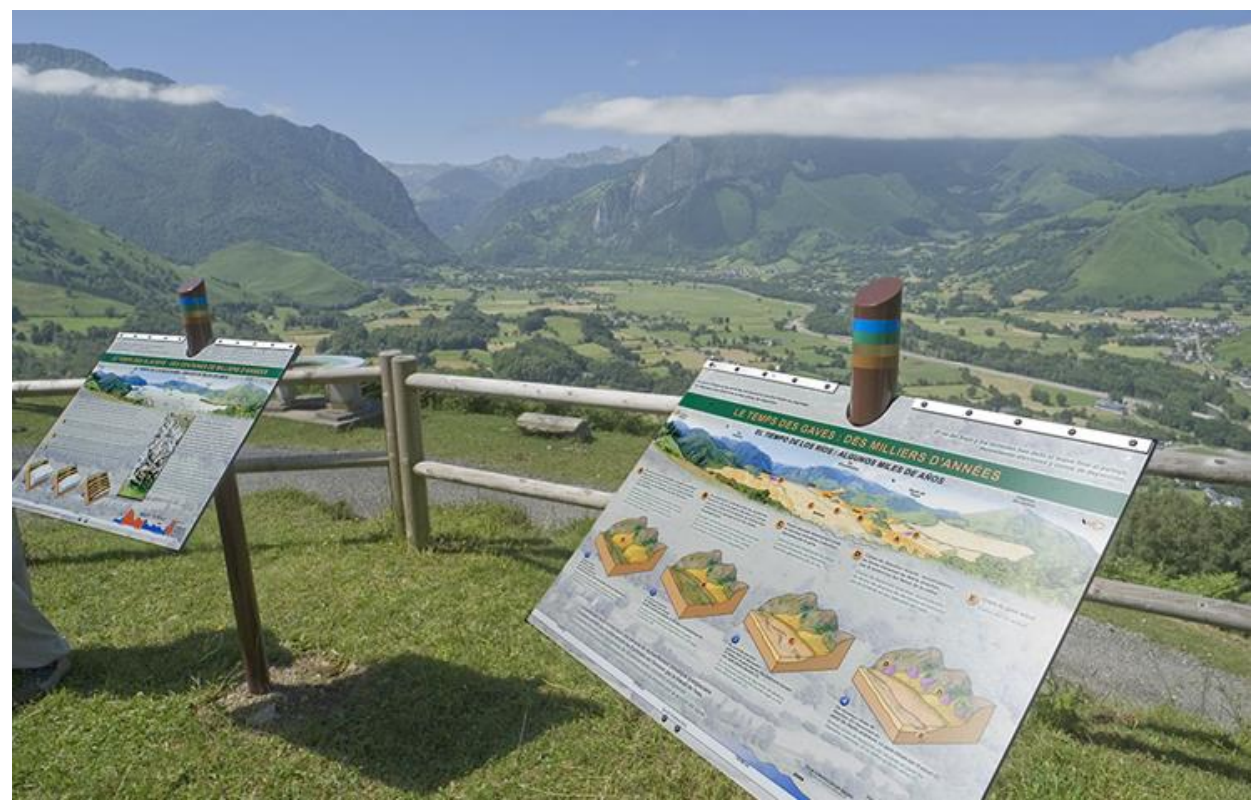

Fig. 36. Example of explanatory signboards made for the Route Géologique Transpyrénéenne.

The proliferation of national and international meetings related to the geological heritage and its touristic development (geotourism) is another effect of the dynamics of the current dynamics of this discipline, including the Declaration of Arouca (Portugal), and geoheritage sessions at many national and international conferences, even including the global International Geological Congress held in Brisbane, Australia in 2012.

Geoheritage can also be used to support local sustainable development and enhancement of a territory by involving the other types of heritage present. This idea is the basis of the concept of Geopark, a label supported by UNESCO. A new Geopark has been recently created in France, which has now five (Ardèche, Chablais, Luberon, Massif des Bauges, Réserve Géologique de Haute Provence).

\section{Valuation of the inventory in international programs}

In 1995, the International Union of Geological Sciences (IUGS) launched a project called Global Geosites to produce an inventory of sites of international relevance for conservation and to build a database of these global geosites, in connection with UNESCO. A working group (Global Geosites Working Group) was then established. But after a few years, the project was shelved by IUGS and UNESCO. In 2010, this project was reactivated; the IUGS created a new working group dedicated to geoheritage (Geoheritage Task Group). One of the purposes of the working group is to develop a database of international geosites (http://geoheritage-iugs.mnhn.fr/).

Geosites identified and validated in France at the national level in the National Inventory program, and considered of international relevance, may, for example, be transferred to the international database, similarly to those already identified in other countries (Netherlands, Portugal, Spain, etc.) 


\section{Conclusion}

Despite an long interest in what we would now call geoheritage (with a first inventory compiled in 1913 by Martel), this topic has been generally poorly regarded in France. For years, only a few people and bodies were making a true effort in geoconservation, through a specific protection programme, the Réserves Naturelles de France.

A decade ago, a law was enacted and put the inventory, and therefore geoheritage in general, on the front stage (proscenium). A methodology was developed through wide collaboration and the inventory was launched some seven years ago. Such an inventory is a prerequisite for the identification and understanding of outstanding geological sites, and to assess their heritage value and their protection needs. But there is also the relevance of this 'new' concept of heritage fir the promotion and development of territories, alongside other more familiar types of heritage (cultural, architectural, industrial, etc.).

The inventory is conducted in such a manner that its data are compatible with other data concerning nature (fauna, flora, etc.). Thus, its results are directly usable by a wide community of geologists, managers and economists for research, education, geoconservation and geotourism - and a large part of the French territory is already covered with the inventory. In conclusion, the absence of an inventory was a handicap in France for a long time, but now that it is established it is revealed as a true advantage, promising a most interesting future for geoheritage across the territory.

Acknowledgements We are happy to thank Gabriel Carlier and François Farges (MNHN) for their contribution to the tables 1, 2 and 3, Lola Johannes for her proof-reading of the table lithotype, Tiphaine Dubreuil (MNHN/SPN) and Alexandre Lethiers (UMR7207/UPMC) for their graphic works, and Pr. Jean-Paul Cadet for reviewing the manuscript. This work was partially financed by ASM Geopatrimoine and the French Ministry in charge of the Environment.

\section{References}

AguedaVillar J A, García-Cortés A,Palacio Suárez-Valgrande, J (Eds)(2009)Spanish geological frameworks and geosites. An approach to Spanish geological heritage of international relevance. Madrid, Espagne: Geological Survey of Spain (IGME). 234 pages. Available at :

http://www.igme.es/internet/patrimonio/GEOSITES/publication.htm

Billet G, Bonnefoy B, De Wever P, Houssaye A, Merle D (2008)Promenade géologique à Etampes. Mèze, Biotope - Paris, MNHN- Orléans, BRGM (Collection Balades géologiques), 28 pages.

BrilhaJ, Andrade C, Azerêdo A, Barriga FJAS, Cachão M, Couto H, Cunha PP, Crispim JA, Dantas P, Duarte LV, Freitas MC, Granja HM, Henriques MH,Henriques P,Lopes L, Madeira J, Matos JMX, Noronha F, Pais J, Piçarra J,Ramalho MM,. Relvas JMRS, 
Ribeiro A, Santos A, Santos VF, and Terrinha (2005)Definition of the Portuguese frameworks with international relevance as an input for the European geological heritage characterisation. Episodes, 28(3), pp.177-186.

Capellini G (1882) Compte rendu de la 2me session, Bologne, 1881, Volume 2 Imprimerie Fava et Garagnani, 1882, 661 pages (Résolutions concernant la nomenclature et les couleurs, votées par le Congrès, in Congrès Géologique International, pp. 196-198).

Caro M,Mulder T (2010) Promenade géologique à Bordeaux. Mèze, Biotope - Paris, MNHN, Orléans, BRGM (Collection Balades géologiques), 36 pages.

Cayla N (2009) Le patrimoine géologique de l'arc alpin. Thèse Université de Chambéry, 303 pages.

Charpentier JFW (1778)MineralogischeGeographie der Chursächsischen Lande. Leipzig.

Colleté C (coord.) (2010) Stratotype Albien. Paris, Muséum national d'Histoire naturelle Mèze, Biotope - Orléans, BRGM, 332 pages (Patrimoine géologique ; 2)

Cuvier G, Brongniart A (1811) Essai sur la géographie minéralogique des environs de Paris, avec une carte géognostique, et des coupes de terrain. Baudouin, Paris, 278 pages.

Czech Geological Survey (2014)Significant geological localities of the Czech Republic.In: Geological localities. Available at: http://lokality.geology.cz/d.pl?item=1\&l=e

De Wever P, Merle D, Bonnefoy B, Billet G (2009) Promenade géologique à Milly-la-Forêt. Mèze, Biotope - Paris, MNHN - Orléans, BRGM, (Collection Balades géologiques), 28 pages

De Wever P, Le Nechet Y, Cornée A (2006) Vade-mecum pour l'inventaire national du patrimoine géologique. Mémoire hors-série de la Société Géologique de France, $162 \mathrm{p}$

Desor E (1847) Sur le terrain danien, nouvel étage de la craie. Bulletin de la Société Géologique de France 2(4), pp.179-182

DoranM (1978) Conversations avec Cézanne, Michael Doran, Maurice Denis, Émile Bernard, Joachim Gasquet, Ambroise Vollard, Gustave Geffroy, Léo Larguier, Jules Borély, Francis Jourdain, R.P. Rivière, Jacques Félix Simon Schnerb, Karl Ernst Osthaus.Edition Macula, Paris, 320 p, page 122.

Dufrénoy PA,EliedeBeaumont L (1841)Carte géologique de la France (exécutée sous la dir. de M. Brochant de Villiers). 1 :500 000, 6 cartes. Ministère des Travaux Publics, Paris

Egoroff G, De Wever P, Merle D, Métivier B (2011) Promenade géologique à Dourdan. Mèze, Biotope - Paris, MNHN (Collection Balades géologiques), 34 pages

Egoroff G, De Wever P, Cornée A, Monod K, (2011)Du Grenelle 1 à la protection du patrimoine géologique. Géochronique, $\mathrm{n}^{\circ} 119$,pp. 17-19.

Ellis N(2011)Geological Conservation Review (GCR) in Great Britain-Rationale and methods. Proceedings of the Geologists' Association, $\mathrm{n}^{\circ}$ 122, pp. 353-362.

Finland's Ministry of Justice(2014)Land Extraction Act. In :Finlex. Available at: http://www.finlex.fi/fi/laki/ajantasa/1981/19810555 (in Finnish).

Finnish Environment Institute (SYKE) (2014)Geologisetmuodostumat. In: Joint website of Finland's environmental administration. Available at: http://www.ymparisto.fi/fiFI/Luonto/Geologiset_muodostumat (in Finnish).

García-Cortés A, Carcavilla L(2009) Documento metodológico para la elaboración del inventario español de lugares de interés geológico (IELIG). Madrid, Spain: Geological Survey of Spain (IGME). 61 p. Available at: http://www.igme.es/patrimonio/novedades/METODOLOGIA\%20IELIG\%20web.pdf

García-Cortés A(2012)Inventaire du patrimoine géologique en Espagne. In :Egoroff, G, Cornée A, De Wever P, Lalanne A (Eds), 2013. Actes du colloque "Géopatrimoine, un lustre d'inventaires en France". 10-12 octobre 2012, Digne les Bains, Mémoires de la Société Géologique de France, hors-série n 13 . pp. 30-39.

García-Cortés A, Carcavilla L, Vegas J, Díaz-Martínez E, (2013)Algunos resultados del Inventario de Lugares de Interés Geológico de la Cordillera Ibérica. In: Vegas J, Salazar 
A, Díaz-Martínez E,Marchán C (eds.): Patrimonio geológico, un recurso para el desarrollo. Cuadernos del Museo Geominero, vol. 15, p. 379-388. IGME, Madrid.

GeoConservationUK (2014). RIGS. Available at:

http://wiki.geoconservationuk.org.uk/index.php5?title=RIGS

Gläser $\quad$ FG (1775)VersucheinermineralogishenBeschreibung der gefürstetenGraffschaftHenneberg. Leipzig.

Graviou P (2012) Géotourisme en côte d'Armor. Coll. Géotourisme, SGMB/BRGM, Ed. Biotope/SGMB, 96 pages

GuetTARDJ-E(1746) Mémoire et carte géologique. Sur la nature des terreins qui traversent la France et l'Angleterre. Mémoires de l'Académie royale des Sciences, Paris, pp.363-393

Hanzo M (coord.) (2012)StratotypeHettangien. Paris, Muséum national d'Histoire naturelle Mèze, Biotope -, 316 pages (Patrimoine géologique; 3)

Husa J,Teeriaho J (2004) Bedrock areas valuable for nature and landscape protection in Uudenmaa. Regional Research Publications $\mathrm{n}^{\circ} 350$. Helsinki (Finland): Finnish Environment Institute. 469 p. (in Finnish).

IGN (2012) Fonds de cartes.In: IGN Education. Available at: http://education.ign.fr/ressources/fonds-de-cartes

IUCN World conservation Congress (2008) Resolution WCC-2008-Res-040 (2008) Barcelona. Available at:

https://portals.iucn.org/library/efiles/documents/WCC-4th-005.pdf

IUCN World Conservation Congress (2012) Resolution WCC-2012-Res-048 (2012) Jeju. Available at:

https://portals.iucn.org/library/efiles/documents/WCC-5th-005.pdf

Joint Nature Conservation Committee (JNCC) (2014).-Geological Conservation Review (GCR).Avalable at: http://jncc.defra.gov.uk/page-2947

Jonin M (2008) Géodiversité en Bretagne. Un patrimoine remarquable.Coll. «Les cahiers naturalistes de Bretagne », Biotope, 160 pages.

Kubalikova L, Kirchner K (2013)Geoconservation in the Czech Republic and geomorphosites assessment for geotourism and geoeducation purposes: A case study from Podyji National Park. IGeoconservation in the Czech Republic and geomorphosites assessment for geotourism and geoeducation purposes: A case study from Podyji National Park. In: HOBLEA F, CAYLA N,REYNARD E, (Eds). Actes du Colloque International «Gestion des géosites dans les espaces protégés », Symposium International sur la gestion des géosites, «ISGM $2011 »$, Savoie-Mont-Blanc, France, 7-10 Septembre 2011. Collection EDYTEM, ${ }^{\circ}{ }^{15}$. pp. 33-40.

Londeix L(coord.) (2014)Stratotype Aquitanien. Paris, Muséum national d'Histoire naturelle Mèze, Biotope, $416 \mathrm{p}$ (Patrimoine géologique ; 5).

Lozouet P (coord.) (2012)Stratotype Stampien. Paris, Muséum national d'Histoire naturelle Mèze, Biotope -, 464 p (Patrimoine géologique; 4)

Martel E A (1913) La question des parcs nationaux en France. Comment et pourquoi créer les parcs nationaux. In La Montagne. Revue mensuelle du Club Alpin français. Août 1913, $24 \mathrm{p}$.

Martel E A (1928)La France ignorée. Tome I : Sud-Est de la France, 290 pages. Availableat: http://jubilotheque.upmc.fr

Martel E A (1932) La France ignorée. Tome II : Des Ardennes aux Pyrénées, 306 pages.

Mason V, Stanley M (Eds)(2000)RIGS Handbook. GeoConservationUK. 308 pages Available at:

http://wiki.geoconservationuk.org.uk/index.php5?title=RIGS_Handbook_Contents 
Merle D (coord.) (2008) StratotypeLutétien. Paris, Muséum national d'Histoire naturelle Mèze, Biotope - Orléans, BRGM, 288 p (Patrimoine géologique; 1)

Natural England (2014) Sites of Special Scientific Interest. Availableon:

http://www.sssi.naturalengland.org.uk/Special/sssi/index.cfm

Page, K.N. and Wimbledon, W.A. 2009. The conservation of Jurassic heritage in the UK - a critical review of current practice and effectiveness. Volumina Jurassica 6: 163-173.

Omalius d'Halloy J-B (1816) Mémoire sur l'étendue géographique du terrain des environs de Paris. Annales des Mines 1, pp.231-266

Robaszynski F,Guyétant G (2009) Des roches aux paysages dans le Nord-Pas-de-Calais. Richesse de notre patrimoine géologique. Société géologique du Nord et Conservatoire des sites naturels du Nord et du Pas-de-Calais, 162 pages.

Schneer, C.J. 1954. The rise of historical geology in the 17th century. Isis, 45 (3), No.141 (Sept. 1954): pp.256-268.

Smith W(1815) A memoir to the map and delineation of the strata of England and Wales. John Cary, London, 51 pages

Société géologique de France (ed.) 1994 Actes du premier symposium international du patrimoine géologique. Digne-les-Bains, 11-16 juin 1991, Mémoire de la Société Géologique de France, 165, 276 pages.

U.N. Educational, Scientific, and Cultural Organization. General Conference, $17^{\text {th }}, 1972$, Convention Concerning the Protection of the World Cultural and Natural Heritage. Paris, 1972

Wimbledon WAP (1996) GEOSITES - A new IUGS initiative to compile a global comparative site inventory, an aid to international and national conservation activity. Episodes 19, pp87-88

Wimbledon WAP, Smith-Meyer S (Eds) (2012) Geoheritage in Europe and its conservation. Oslo (Norway): ProGEO, 405 pages. 
Appendix 1: The French law enacting the inventory of geological inventory: Article L411-5 du code de l'environnement.

http://www.legifrance.gouv.fr/affichCodeArticle.do;jsessionid=366CEC157AF9A6141272A5 72476FA595.tpdjo14v_1?cidTexte=LEGITEXT000006074220\&idArticle=LEGIARTI00002 2495736\&dateTexte=20120831\&categorieLien=id\#LEGIARTI000022495736

I. - L'inventaire du patrimoine naturel est institué pour l'ensemble du territoire national terrestre, fluvial et marin. On entend par inventaire du patrimoine naturel l'inventaire des richesses écologiques, faunistiques, floristiques, géologiques, minéralogiques et paléontologiques.

L'Etat en assure la conception, l'animation et l'évaluation. Les régions peuvent être associées à la conduite de cet inventaire dans le cadre de leurs compétences. En outre, les collectivités territoriales peuvent contribuer à la connaissance du patrimoine naturel par la réalisation d'inventaires locaux, ayant notamment pour objet de réunir les connaissances nécessaires à l'élaboration du schéma régional de cohérence écologique mentionné à l'article L. 371-3.

Le préfet de région, les préfets de départements et les autres collectivités territoriales concernées sont informés de ces élaborations.

Ces inventaires sont conduits sous la responsabilité scientifique du Muséum national d'histoire naturelle.

Lors de l'élaboration d'un plan, programme ou projet, le préfet communique à la commune ou à l'établissement public de coopération intercommunale compétent toutes informations contenues dans ces inventaires utiles à cette élaboration.

II. - Les dispositions de la loi du 29 décembre 1892 sur les dommages causés à la propriété privée par l'exécution des travaux publics sont applicables à l'exécution des opérations nécessaires à la conduite de ces inventaires. Ces dispositions sont également applicables à la connaissance du sol, de la végétation et de tout renseignement d'ordre écologique sur les territoires d'inventaires.

III. - Il est institué dans chaque région un conseil scientifique régional du patrimoine naturel. Ce conseil est constitué de spécialistes désignés intuitu personae pour leur compétence scientifique, en particulier dans les universités, les organismes de recherche, les sociétés savantes, les muséums régionaux. Il couvre toutes les disciplines des sciences de la vie et de la terre pour les milieux terrestres, fluviaux et marins.

Ses membres sont nommés par arrêté du préfet de région après avis du président du conseil régional.

Il élit en son sein un président.

Il peut être saisi pour avis par le préfet de région ou le président du conseil régional sur toute question relative à l'inventaire et à la conservation du patrimoine naturel.

Un décret en Conseil d'Etat définit sa composition, ses domaines d'intervention et précise les conditions dans lesquelles il est saisi. 
Appendix2: An example of information provided for a site belonging to the inventory. A typical report has between 3 and 10 pages,at least one ma, and several pictures.
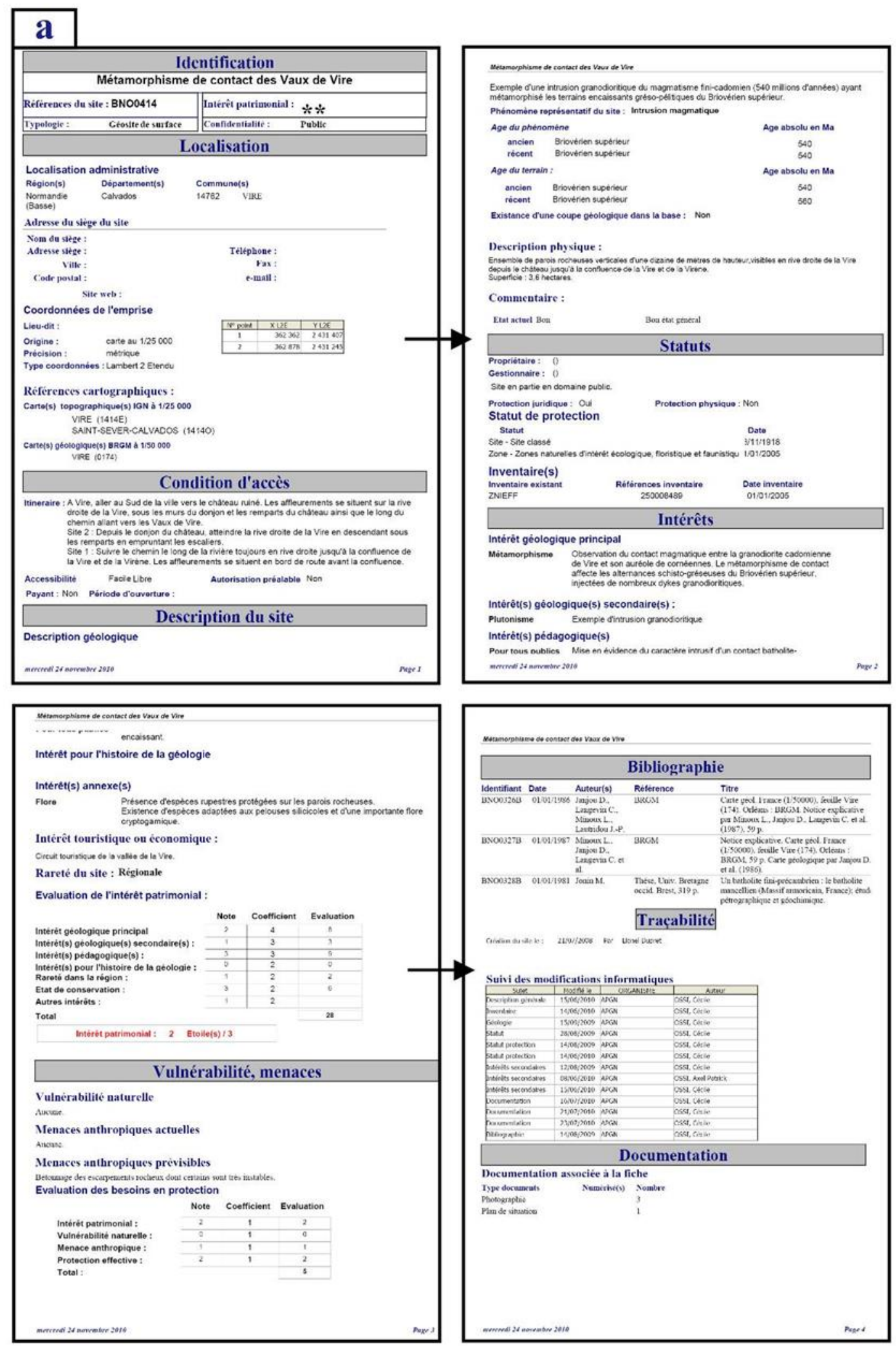

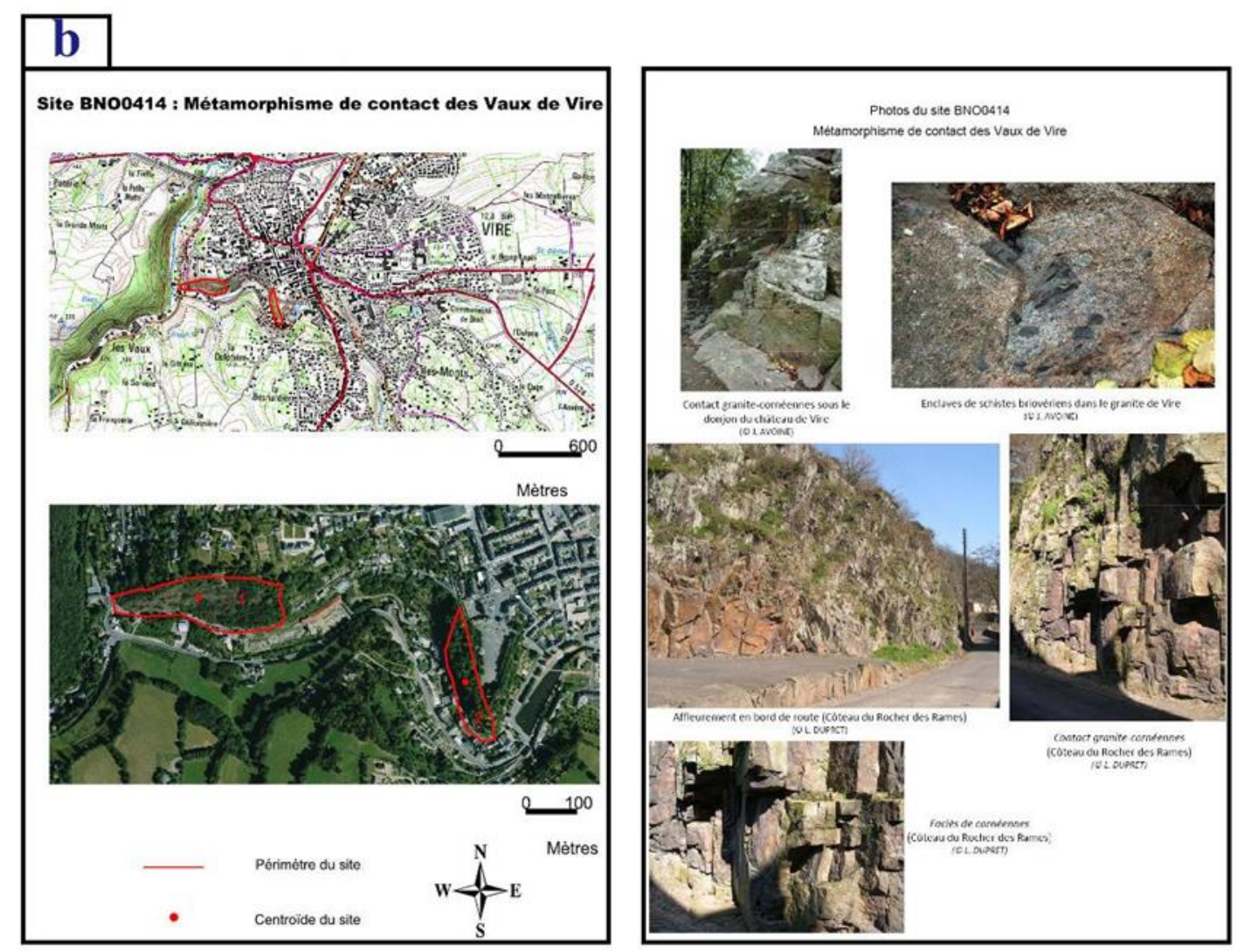
Appendix3: Internet sources for state geosite inventories in Europe.

Czech Republic

Czech Geological Survey(2014). Significant geological localities of the Czech Republic: http://www.geology.cz/extranet-eng/geology-for-all/geological-localities

Denmark

Geological Survey of Denmark(2006). Geosites in Denmark: http://geosites.dk/

Estonia

Geological Survey of Estonia (2014).Estonian Geosites: http://www.egk.ee/aboutgse/geological-treasures/geosites/?lang=en

Finland

Finnish Environment Institute (SYKE) (2014). National Inventory of geological formations: http://www.ymparisto.fi/fi-FI/Luonto/Geologiset_muodostumat (in Finnish)

$\underline{\text { Ireland }}$

Geological Survey of Ireland (2014). Irish Geological Heritage Program: http://www.gsi.ie/Programmes/Heritage+and+Planning/

$\underline{\text { Italy }}$

Environmental protection and research institute (ISPRA) (2009). The Geosites database: http://sgi2.isprambiente.it/geositiweb/

Lithuania

Lithuanian Geological Survey (2011).Geotops database: http://www.lgt.lt/index.php?page=33\&mod_id=69\&action=showFull\&id=219\&lang=en

Netherlands

Netherlands Organisation for Applied Scientific Research (TNO) (2014).Geosites in Netherlands: http://www.geosites.nl/ (in Dutch)

$\underline{\text { Poland }}$

Institute of Nature Conservation of the Polish Academy of Sciences (2012).Database of Polish Representative Geosites: http://www.iop.krakow.pl/geosites

$\underline{\text { Slovakia }}$

State Geological Institute of Dionýz Štúr (State Geological Survey of the Slovak Republic) (2014).Important Geological Sites: http://mserver.geology.sk:8085/g_vgl/?jazyk=EN

$\underline{\text { Spain }}$

Geological Survey of Spain (IGME) (2014).Spanish inventory of sites of geological interest: http://info.igme.es/ielig/ (in Spanish).

$\underline{\text { Switzerland }}$

Lausanne University Geography Institute (IGUL) (2010). Geosites of national importanceswiss inventory: http://mesoscaphe.unil.ch/geodata/geosites2/

$\underline{\text { United-Kingdom }}$ 
Joint Nature Conservation Committee (2008). Geological Conservation review: http://jncc.defra.gov.uk/page-2947 